\title{
A naturally light Higgs without light top partners
}

\author{
Adrián Carmona ${ }^{a}$ and Florian Goertz ${ }^{a, b}$ \\ ${ }^{a}$ Institute for Theoretical Physics, ETH Zurich, \\ 8093 Zurich, Switzerland \\ ${ }^{b}$ Theory Division, CERN, \\ 1211 Geneva 23, Switzerland \\ E-mail: carmona@itp.phys.ethz.ch, florian.goertz@cern.ch
}

ABStRact: We demonstrate that the inclusion of a realistic lepton sector can relax significantly the upper bound on top partner masses in minimal composite Higgs models, induced by the lightness of the Higgs boson. To that extend, we present a comprehensive survey of the impact of different realizations of the fermion sectors on the Higgs potential, with a special emphasis on the role of the leptons. The non-negligible compositeness of the $\tau_{R}$ in a general class of models that address the flavor structure of the lepton sector and the smallness of the corresponding FCNCs, can have a significant effect on the potential. We find that, with the $\tau_{R}$ in the symmetric representation of $\mathrm{SO}(5)$, an increase in the maximally allowed mass of the lightest top partner of $\gtrsim 1 \mathrm{TeV}$ is possible for minimal quark setups like the $\mathrm{MCHM}_{5,10}$, without increasing the tuning. A light Higgs boson $m_{H} \sim(100-200) \mathrm{GeV}$ is a natural prediction of such models, which thus provide a new setup that can evade ultralight top partners without ad-hoc tuning in the Higgs mass. Moreover, we advocate a more minimal realization of the lepton sector than generally used in the literature, which still can avoid light partners due to its contributions to the Higgs mass in a different and very natural way, triggered by the seesaw mechanism. This allows to construct the most economical $\mathrm{SO}(5) / \mathrm{SO}(4)$ composite Higgs models possible. Using both a transparent 4D approach, as well as presenting numerical results in the 5D holographic description, we demonstrate that, including leptons, minimality and naturalness do not imply light partners. Leptonic effects, not considered before, could hence be crucial for the viability of composite models.

Keywords: Beyond Standard Model, Heavy Quark Physics, Technicolor and Composite Models

ARXIV EPRINT: 1410.8555 


\section{Contents}

1 Introduction 1

2 General structure of the Higgs potential in MCHMs and light partners 4

2.1 Generic (4D) setup of the models 5

$\begin{array}{ll}2.2 & \text { The Higgs potential and light partners } \\ \end{array}$

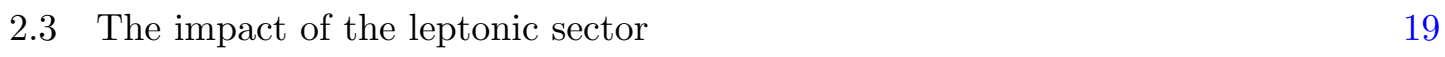

3 Numerical analysis in the GHU approach - the impact of leptons $\quad \mathbf{2 5}$

3.1 Setup of the (5D) GHU models 25

$\begin{array}{lll}3.1 .1 & \mathrm{MCHM}_{5} & 27\end{array}$

$\begin{array}{lll}3.1 .2 & \mathrm{MCHM}_{10} & 30\end{array}$

3.1.3 $\mathrm{MCHM}_{14}, \mathrm{MCHM}_{5-14}, \mathrm{MCHM}_{14-1} \quad 30$

3.1.4 The mMCHM ${ }^{\mathrm{III}}$ : a new minimal model for leptons 33

3.2 The one-loop Higgs mass 34

3.2.1 The Higgs mass at $f_{\pi} \quad 34$

3.2.2 Coleman-Weinberg potential in KK theories 35

3.2.3 The Higgs mass in the MCHMs 36

3.3 Lifting light partners with leptons: numerical results and discussion $\quad 37$

$\begin{array}{lll}3.3 .1 & \text { Minimal quark setups } & 39\end{array}$

3.3.2 The impact of leptons 44

4 Conclusions $\quad 54$

A Generators and explicit form of Goldstone matrix $\quad 56$

B Fermion representations $\quad 56$

$\begin{array}{lll}\text { B.1 Fundamental } & 56\end{array}$

$\begin{array}{lll}\text { B.2 Adjoint } & 57\end{array}$

$\begin{array}{lll}\text { B.3 Symmetric } & 57\end{array}$

\section{Introduction}

The LHC and its experiments have already delivered an outstanding contribution to our understanding of electroweak symmetry breaking (EWSB). With the discovery of the $125 \mathrm{GeV}$ scalar $[1,2]$ and the first determination of its properties, we are lead to the conclusion that a Higgs sector is responsible for EWSB. However, the question if this sector can be identified with the one appearing in the Standard Model (SM) of particle physics is still to be answered. There are various reasons to expect new particles beyond the SM (BSM) and 
then naturalness calls for a mechanism to avoid the sensitivity of the Higgs mass to large scales. Supersymmetric models or models of compositeness, like the most studied minimal composite Higgs models $\mathrm{MCHM}_{5}$ and $\mathrm{MCHM}_{10}$, provide an elegant incarnation of such a protection mechanism. Both ideas assume the presence of BSM physics not far above the electroweak scale $M_{\mathrm{EW}} \sim v$ in order to avoid the quadratic ultra-violet (UV) sensitivity of the Higgs mass but rather saturate it in the infra-red (IR). For the latter class of models, the separation between the Higgs mass and the BSM scale, where new resonances appear, can naively be larger since the Higgs is realized as a pseudo Goldstone boson of the coset $\mathrm{SO}(5) / \mathrm{SO}(4)$, providing an additional protection for its mass.

However, in the MCHMs mentioned before, the fact that the Higgs boson is rather light, $m_{H} \approx 125 \mathrm{GeV}$, requires the presence of light partners of the top quark [3-11]. This tendency can be easily understood from the fact that generically the linear mixing terms between the top quark and the composite fermionic partners, needed to generate the top mass via the concept of partial compositeness, break the Goldstone symmetry and thus contribute to the Higgs mass. The large value of the top mass requires the masses of the top partners to be rather small in order to generate a large mixing with the composite sector without introducing too large coefficients of the linear mixing terms, that would make the Higgs too heavy. So in both classes of solutions to the naturalness problem one expects top partners at $\lesssim \mathcal{O}(1) \mathrm{TeV}$. The non-observation of these particles at the LHC so far has already put both ideas under some pressure.

For the $\mathrm{MCHM}_{5,10}$, where the composite fermions are realized in fundamental and adjoint representations of $\mathrm{SO}(5)$, respectively, the presence of light partners significantly below the actual scale of these models has explicitly been demonstrated in [3,5-10]. ${ }^{1}$ For a $125 \mathrm{GeV}$ Higgs boson their masses have been shown to lie around $m_{\tilde{t}}^{(5)} \sim 600 \mathrm{GeV}$ and $m_{\tilde{t}}^{(10)} \sim 400 \mathrm{GeV}$, given that the fundamental mass scale of the models resides at the $\mathrm{TeV}$ scale, as suggested by naturalness. This is even in a region probed currently by experiments at the LHC and provides an option to discover signs of these models, but in the case of no observation also is a potential threat to the composite Higgs idea. Indeed, the $\mathrm{MCHM}_{10}$ is already severely challenged by top partner searches, see also section 3 .

The only viable way out of the necessity of such ultra-light states found so far, requires the embedding of quarks in a symmetric representation 14 of $\mathrm{SO}(5)$. These models however suffer generically from an ad-hoc tuning $[9,10]$. While in the $\mathrm{MCHM}_{5,10}$ after EWSB the Higgs mass is automatically generated not too far from the experimental value, and light fermionic partners offer the option to arrive at $m_{H}=125 \mathrm{GeV}$, this is not true for this realization with a 14. In contrast to the other models, one needs a sizable tuning of in general unrelated quantities to arrive at the correct Higgs mass, which is naturally predicted much too heavy, $m_{H}^{(14)} \sim 1 \mathrm{TeV}$. This is to be contrasted with the "double tuning" in the $\mathrm{MCHM}_{5,10}$, which is required to achieve a viable EWSB [7], see section 2.2. Thus, while evading the necessary presence of problematic fermionic partners, the attractive prediction of a generically light Higgs boson is challenged and one needs to induce a different

\footnotetext{
${ }^{1}$ We will not consider the spinorial representation 4, as in the fermion sectors where it could have an impact on our analysis it is not viable due to a lack of protection for $Z \bar{b}_{L} b_{L}$ (or $Z \bar{\tau}_{R} \tau_{R}$ ) couplings.
} 
kind of ad-hoc cancellation, which is not linked to the necessity of a suitable EWSB, but rather to the particular experimental value of the Higgs mass, not favored by the model. While, besides that, there exist viable setups featuring a symmetric representation of $\mathrm{SO}(5)$, one should also note that models like the $\mathrm{MCHM}_{14}$ suffer from a large modification of $\sigma(g g \rightarrow H)$ and are already disfavored from Higgs physics at the LHC. Moreover, the setups considerably enlarge the particle content and parameter space with respect to the most minimal realizations without an obvious structural reason.

In this article, we will introduce a new class of options to lift the mass of the top partners. They can realize a light Higgs without an ad-hoc tuning and without large changes in Higgs production, thus adding new aspects to the question if the non-observation of fermionic partners below the $\mathrm{TeV}$ scale together with at most modest effects in Higgs production already put the $\mathrm{SO}(5) / \mathrm{SO}(4)$ composite Higgs framework under strong pressure. To that extend we analyze the influence of leptons on the Higgs potential. Naively, such contributions seem not to be relevant due to the small masses of the leptons, leading at first sight to a small mixing with the composite sector and thus a small Goldstone-symmetry breaking. However, in a general class of models that address the flavor structure of the lepton sector (e.g. via flavor symmetries) and in particular the smallness of leptonic flavorchanging neutral currents (FCNCs), there is a natural suppression of the Yukawa couplings in the composite sector as well as of the left-handed lepton compositeness (see [12]). The reason for the former is that the Yukawa couplings control the size of the breaking of the flavor symmetries. The latter (more relevant) suppression is due to the fact that the left handed lepton couplings are in general not protected by custodial symmetry. Potentially dangerous corrections thus need to be suppressed by the elementary nature of the lefthanded leptons. In these flavor-protected models, the $\tau_{R}$ needs to mix stronger with the composite sector than naively expected, in order to generate its non negligible mass, which can lead to interesting effects in the Higgs potential. ${ }^{2}$

Beyond that, we will point out a new motivation for charged lepton compositeness, built on the mere size of the neutrino masses. As it will turn out, the most minimal realization of the type-III seesaw mechanism in the composite Higgs framework projects the modest IR localization of right-handed neutrinos onto the charged leptons. The possible Majorana character of neutrinos thus leads to distinct new features in the lepton sector, compared to the light quarks.

It turns out that the contributions of the $\tau$ sector to the Higgs mass can interfere destructively with the top contribution and, for the symmetric $\mathrm{SO}(5)$ representation, can lift the masses of the light fermionic resonances significantly above the region of $m_{\tilde{t}} \lesssim$ $1 \mathrm{TeV}$, currently tested at the LHC, even if the quark sector corresponds to the $\mathrm{MCHM}_{5}$ or $\mathrm{MCHM}_{10}$. Thus, such potential contributions should be taken into account when examining

\footnotetext{
${ }^{2}$ Note that this is not the case in the bottom sector, where a relatively large degree of compositeness of the doublet component $b_{L}$ is required due to the large top-quark mass, in turn not allowing for a sizable mixing of the $b_{R}$ with the composite sector (where the latter also in general features no custodial protection). On the other hand, models of $\tau_{R}$ compositeness feature in general also enough protection of the $\tau_{R}$ couplings, not to be in conflict with precision tests [13] and can have an interesting (possibly modest) impact on Higgs physics at the LHC [14].
} 
the viability of the composite Higgs idea. As we will show, lifting the top partner masses via leptonic contributions has several intriguing features. First, one opens the possibility to avoid the large ad-hoc tuning appearing in equivalent realizations via the quark sector in a way that ultra-light resonances are even disfavored from the point of view of the tuning. Moreover, we will show that it is possible to evade light partners even without abandoning the concept of minimality of the setup.

This article is organized as follows. In section 2 we provide a complete survey of possible realizations of the fermion sector of the composite setup for the chiral SM-fermions mixing with any one of the basic representations of $\mathrm{SO}(5)$ up to a $\mathbf{1 4}$ and discuss the structure of the Higgs potential and its mass via a spurion analysis. In particular, we review the emergence of light partners and demonstrate in a general way how they could be avoided via leptonic contributions, pointing out the virtues of this approach. While in this section we follow a generic, particular suited, 4D approach that makes the important mechanisms more transparent, in section 3 we will give explicit numerical results in the Gauge-Higgs unification (GHU) setup, which provides a weakly coupled dual description of the composite Higgs idea. Here, we will confirm the general findings of the previous section and provide detailed results for the top partner masses and the tuning, in dependence on the Higgs mass for all important incarnations of the fermion sector. In particular, we introduce a new realization of the lepton sector in GHU models, embedding both the charged and neutral leptons in a single $\mathbf{5}_{L}+\mathbf{1 4}_{R}$, thus working with less degrees of freedom than in the standard $\mathrm{MCHM}_{5}$-like setup and a significantly reduced number of parameters. Employing a typeIII seesaw mechanism, we make explicitly use of the additional $\mathrm{SU}(2)_{L}$ triplets provided by the $\mathbf{1 4}_{R}$, presenting the most minimal composite model with such a mechanism - which even allows for an enhanced minimality in the quark sector with respect to known models. This avoids many additional colored states at the $\mathrm{TeV}$ scale and allows for the least number of new degrees of freedom of all known viable setups of $\mathrm{SO}(5) / \mathrm{SO}(4)$. We will show that this very minimal realization of leptons just belongs to the class of models that has the strongest impact on the Higgs mass and demonstrate its capabilities for lifting the light partners. We finally conclude in section 4 .

\section{General structure of the Higgs potential in MCHMs and light partners}

In this section, we review the structure of the Higgs potential in composite models and the emergence of "anomalously" light top partners in models with a naturally light Higgs boson. We will then illustrate how including the effects from a realistic lepton sector allows to construct models that evade the necessary presence of fermion partners with masses $\lesssim 1 \mathrm{TeV}$, without introducing a large (ad hoc) tuning. To make the important physics more transparent, while keeping the discussion as generic as possible, we will work with general $4 \mathrm{D}$ realizations of the composite Higgs framework [6, 7, 15-18] and later provide the connection to the dual 5D GHU setup [19-24], which adds explicit calculability to the strongly coupled $4 \mathrm{D}$ models. 


\subsection{Generic (4D) setup of the models}

In composite Higgs models, the Higgs field is assumed to be a composite state of a new strong interaction. In consequence, corrections to the Higgs mass are cut off at the compositeness scale such that it is saturated in the IR. Moreover, following the analogy with the pions in QCD, it is generically realized as a pseudo Nambu-Goldstone boson (pNGB) associated to the spontaneous breaking of a global symmetry [25-33], see also [23, 24]. This provides a natural reasoning for the fact that the Higgs is lighter than potential new resonances of the models. The minimal viable breaking pattern featuring a custodial symmetry for the $T$ parameter is $\mathrm{SO}(5) \rightarrow \mathrm{SO}(4)$, which leads to four Goldstone degrees of freedom. The pNGB Higgs can thus be described by the real scalar fields, $\Pi_{\hat{a}}, \hat{a}=1, \ldots, 4$, embedded in the $\Sigma$ field

$$
\Sigma=U \Sigma_{0}, \quad U=\exp \left(i \frac{\sqrt{2}}{f_{\pi}} \Pi_{\hat{a}} T^{\hat{a}}\right),
$$

which transforms in the fundamental representation of $\mathrm{SO}(5)$. Here, $\Sigma_{0}=\left(0,0,0,0, f_{\pi}\right)^{T}$ specifies the vacuum configuration, preserving $\mathrm{SO}(4), f_{\pi}$ is the pNGB-Higgs decay constant and $T^{\hat{a}}$ are the broken generators belonging to the coset $\mathrm{SO}(5) / \mathrm{SO}(4)$. These generators are defined in appendix A, together with the remaining $\mathrm{SO}(4) \cong \mathrm{SU}(2)_{L} \times \mathrm{SU}(2)_{R}$ generators $T_{L}^{a}, T_{R}^{a}$.

Under $g \in \mathrm{SO}(5)$, the Goldstone matrix $U$ appearing in the decomposition (2.1) transforms as $[34,35]$

$$
U \rightarrow g \cdot U \cdot \hat{h}^{T}(\Pi, g), \quad \hat{h}=\left(\begin{array}{cc}
\hat{h}_{4} & 0 \\
0 & 1
\end{array}\right), \quad \hat{h}_{4} \in \mathrm{SO}(4),
$$

such that $\Sigma \rightarrow g \cdot \Sigma$. The above construction provides a non-linear realization of the $\mathrm{SO}(5)$ symmetry on the $\Pi$ fields, which however transform in the fundamental representation of the unbroken $\mathrm{SO}(4)$ (i.e., as a bi-doublet under $\left.\mathrm{SU}(2)_{L} \times \mathrm{SU}(2)_{R}\right)$. Finally note that the $\Sigma$ field just corresponds to the last column of the Goldstone matrix $U, \Sigma=f_{\pi} U_{I 5}$. The fact that the Higgs is realized as a Goldstone of $\mathrm{SO}(5) / \mathrm{SO}(4)$ leads to a vanishing potential at the tree level. Explicit $\mathrm{SO}(5)$-breaking interactions then generate it at one loop, which induces a Higgs vacuum expectation value (vev) $v$, taken along the scalar component $\Pi_{\hat{4}} \equiv h=H+v$, with $\langle H\rangle=0$, mediating EWSB.

Beyond the pNGB Higgs, composite models generically contain fermionic and bosonic resonances with masses $m_{\Psi, \rho} \sim g_{\Psi, \rho} f_{\pi} \lesssim 4 \pi f_{\pi}$, bound states of the new strong sector and transforming via $g$, in addition to the elementary SM-like fields. These bound states can be resolved only beyond a scale $\Lambda \sim 4 \pi f_{\pi} \gg m_{H}$, that defines the cutoff of the pNGB model. Since the effect of the gauge resonances is of minor importance for our study, we will neglect them in the following, see below. Moreover, for our discussion of the Higgs potential only those fermionic resonances are important that appear in the breaking of the global $\mathrm{SO}(5)$ symmetry via large linear mixings to the SM, mediating the masses generated in the composite sector to the SM fields, as detailed below. They correspond to leading approximation just to the composite partners of the (up-type) third generation quarks $t_{R}$, 
$q_{L}$, as well as of the $\tau_{R}$. Note that these excitations contain in particular fields that are significantly lighter than the general scale of the new resonances, $m_{\text {cust }} \lesssim f_{\pi} \ll m_{\Psi, \rho}$, dubbed light custodians, as for the models we consider these modes are present due to custodial symmetry [3, 6, 36-42]. These fields will be of special importance for the Higgs potential. In consequence of the above discussion, in this section we will consider an effective (low energy) realization of the composite setup, including only the resonances associated to the third generation top and $\tau$ sectors, to study the impact of different incarnations of the fermion sector on the Higgs potential, while the other resonances are integrated out at zeroth order. ${ }^{3}$ Finally, note that a generally subleading contribution to the potential still remains present inevitably from weakly gauging just the diagonal SM electroweak subgroup $G_{\mathrm{EW}}=\mathrm{SU}(2)_{L} \times \mathrm{U}(1)_{Y}$ of the global composite $\mathrm{SO}(5) \times \mathrm{U}(1)_{X}$ and elementary $\mathrm{SU}(2)_{L}^{0} \times \mathrm{U}(1)_{R}^{0}$ groups, which also explicitly breaks the $\mathrm{SO}(5)$ symmetry. ${ }^{4}$

Neglecting subleading effects due to the heavy resonances residing at the scale $m_{\rho, \Psi}$ and not associated to the third generation fermions (and as such in particular irrelevant for our discussion of the Higgs potential), our setup is thus described by the low-energy Lagrangian

$$
\mathcal{L}=\mathcal{L}^{\mathrm{SM}}\left[V^{\mu}, f\right]+\mathcal{L}_{\Sigma}+\mathcal{L}_{\text {kin }}^{\Psi}+\mathcal{L}_{\text {mass }}^{\Psi}-V(\Pi)
$$

Here, the $\sigma$-model term $\mathcal{L}_{\Sigma}=\frac{1}{2}\left(D_{\mu} \Sigma\right)^{T} D^{\mu} \Sigma=\frac{f_{\pi}^{2}}{2}\left(D_{\mu} U_{I 5}\right)^{T} D^{\mu} U_{I 5}$ contains the couplings of the composite Higgs to the SM gauge fields via the covariant derivative $D_{\mu}=$ $\partial_{\mu}-i g^{\prime} Y B_{\mu}-i g T^{i} W_{\mu}^{i}$, with $g$ and $g^{\prime}$ the SM gauge couplings. Note that the non-linearity of the Higgs sector, see (2.1), induces a shift in the couplings of order $v^{2} / f_{\pi}^{2}$ with respect to the SM. Moreover, $\mathcal{L}_{\text {kin }}^{\Psi}=\sum_{f=T, t, \mathcal{T}, \tau} \operatorname{Tr}\left[\bar{\Psi}^{f} \gamma^{\mu} D_{\mu} \Psi^{f}\right]$ are the kinetic terms of the composite fermions, each associated to a chiral SM fermion, see below, and $\mathcal{L}^{\mathrm{SM}}\left[V^{\mu}, f\right]$ encodes the spin 1 and spin 1/2 part of the SM Lagrangian containing only vector and/or fermion fields, i.e., the field strength tensors and the covariant derivatives between SM-fermion bilinears (plus gauge fixing and ghost terms).

\footnotetext{
${ }^{3}$ We will comment on the effect of partners of lighter fermions in one case where they might become relevant numerically later.

${ }^{4}$ Switching off the SM gauge interactions and the linear fermion mixings, the Lagrangian is invariant under separate global symmetries in the elementary and composite sectors. The additional $\mathrm{U}(1)_{X}$ factor is needed to arrive at the hypercharges of the SM-fermions, via $Y=T_{R}^{3}+X$, and we omit $\mathrm{SU}(3)_{c}$.

Note that in a full two-site description, including composite gauge resonances, an additional $\sigma$-field $\Omega$ breaks the elementary $\mathrm{SO}(5)^{0}$ (with $\mathrm{SU}(2)_{L}^{0} \times \mathrm{U}(1)_{R}^{0}$ gauged) at the first site and the composite, completely gauged, $\mathrm{SO}(5)$ at the second site to the diagonal subgroup $\mathrm{SO}(5)_{V}[6]$ (see also [17]). One linear combination of the bosons corresponding to the $\mathrm{SU}(2)_{L}^{0} \times \mathrm{U}(1)_{R}^{0}$ and $\mathrm{SU}(2)_{L} \times \mathrm{U}(1)_{R}$ subgroups remains massless, furnishing $G_{\mathrm{EW}}$, while the orthogonal combination and the coset $\mathrm{SO}(5) /\left(\mathrm{SU}(2)_{L} \times \mathrm{U}(1)_{R}\right)$ gauge fields acquire a mass at the scale $m_{\rho}$. The $\Sigma$ Goldstone bosons also contributes to the latter masses since $\Sigma$ breaks $\mathrm{SO}(5) \rightarrow \mathrm{SO}(4)$. A linear combination of $\Omega$ and $\Sigma$ then actually provides the pseudo-Goldstone Higgs, which then delivers the longitudinal degrees of freedom for the massless SM-like gauge fields in a second step. Since the gauge resonances have only a minor impact on the Higgs potential (determined by the weak gauge coupling), here we study the limit of a large $\Omega$ decay constant for simplicity, which decouples all the heavy gauge resonances and leads to the Higgs sector just originating from $\Sigma$.
} 
The most relevant terms for our following discussion appear in the second row. Of particular importance is the fermion mass and mixing Lagrangian

$$
\begin{aligned}
\mathcal{L}_{\text {mass }}^{\Psi}= & \operatorname{Tr}\left\{-\sum_{f, f^{\prime}=T, t} m_{\Psi}^{f f^{\prime}} \bar{\Psi}_{L}^{f} \Psi_{R}^{f^{\prime}}-f_{\pi} \sum_{\substack{f, f^{\prime}=T, t \\
i}} Y_{i}^{f f^{\prime}} \bar{\Psi}_{L}^{f} \cdot g_{i}^{f f^{\prime}}\left(\Sigma / f_{\pi}\right) \cdot \Psi_{R}^{f^{\prime}}\right. \\
& \left.-y_{L}^{t} f_{\pi} \bar{q}_{L} \Delta_{L}^{t} \Psi_{R}^{T}-y_{R}^{t} f_{\pi} \bar{t}_{R} \Delta_{R}^{t} \Psi_{L}^{t}\right\}+ \text { h.c. }+(T \rightarrow \mathcal{T}, t \rightarrow \tau, q \rightarrow \ell),
\end{aligned}
$$

where $m_{\Psi}^{f f^{\prime}}$ are the vector-like masses (and mass mixings) of the fermion resonances and the symbols $\bar{\Psi}_{L}^{f} \cdot g_{i}^{f f^{\prime}}\left(\Sigma / f_{\pi}\right) \cdot \Psi_{R}^{f^{\prime}}, i=1, \ldots, n$ denote all $\mathrm{SO}(5)$ invariant combinations that can be formed out of the bilinear $\bar{\Psi}_{L}^{f} \Psi_{R}^{f^{\prime}}$ and non-trivial functions of $\Sigma$ as well as, possibly, traces. The form of these Yukawa couplings in the strong sector, with coefficients $Y_{i}^{f f^{\prime}}$, depends on the particular representation chosen for the composite fermions. Embedding for example all fermionic resonances in fundamental representations of $\mathrm{SO}(5)$, as in the $\mathrm{MCHM}_{5}$, we obtain

$$
\bar{\Psi}_{L}^{f} \cdot g_{1}^{f f^{\prime}}\left(\Sigma / f_{\pi}\right) \cdot \Psi_{R}^{f^{\prime}} \stackrel{\mathrm{MCHM}_{5}}{\longrightarrow} \bar{\Psi}_{L}^{f} \frac{\Sigma \Sigma^{T}}{f_{\pi}^{2}} \Psi_{R}^{f^{\prime}} \quad(n=1),
$$

and the global trace becomes trivial.

At this point, some comments are in order. The Lagrangian (2.4) is constructed in the most general way that respects the global $\mathrm{SO}(5)$ symmetry, up to linear mixing terms of the SM-like fermions with the composite resonances, where the former transform under $\mathrm{SU}(2)_{L}^{0} \times \mathrm{U}(1)_{R}^{0}$ and not $\mathrm{SO}(5)$. These elementary-composite mixings are parametrized by $y_{L, R}^{t, \tau}$, where the coupling matrices $\Delta_{L, R}^{t, \tau}$ are fixed by gauge invariance under $G_{\mathrm{EW}}$, i.e., they couple appropriate linear combinations of the components of the composite operators with definite charges under the SM gauge group to the corresponding SM fields, see below. After rotating to the mass basis only these bilinear terms induce masses for the SM-like fermions, which now feature composite components proportional to $y_{L, R}^{t, \tau}$, realizing the concept of partial compositeness $[23,24,43]$. The chiral fermion masses are finally proportional to these mixings as well as to the Yukawa coupling in the strong sector, mediating the transition between different components of the $\mathrm{SO}(5)$ multiplets, $m_{t} \sim\left|y_{L}^{t} f_{\pi} / m_{\Psi}^{T T} Y_{i}^{T t} v f_{\pi} / m_{\Psi}^{t t} y_{R}^{t}\right|$, and analogously for the lepton sector. Note that in the holographic $5 \mathrm{D}$ realization of this setup (see section 3), only the off-diagonal Yukawa couplings $Y_{i}^{T t}$ are non zero, while the mass-mixing $m_{\Psi}^{t T}$ vanishes due to boundary conditions. The same is true in the corresponding deconstructed 2-site models, like studied here, if one requires finiteness of the Higgs potential $[6,44]$.

As mentioned, the Lagrangian contains two vector-like resonances $\Psi^{T, t}\left(\Psi^{\mathcal{T}, \tau}\right)$ in each sector, associated to the two chiralities of SM fermions. In the (broken) conformal field theory picture, which turns out to be indeed dual to a 5D (GHU) picture [23, 45-49], the linear-mixing parameters $y_{L, R}^{t, \tau}$ just correspond to the anomalous dimensions of the composite operators that excite the resonances that the SM-like fermions couple to [5053]. Following this line of reasoning, in general each elementary chiral fermion couples to its own resonance (the Lagrangian described above can always be brought to such a basis). 
Note that if the $q_{L}$ and the $t_{R}$ mix with composites that belong to different representations of $\mathrm{SO}(5)$, the vector-masses mixing those composites obviously vanish, $m_{\Psi}^{f f^{\prime}}=0$ for $f \neq$ $f^{\prime}$. Moreover, we have neglected the allowed terms $\sim\left|y_{L, R}^{t}\right| f_{\pi} \bar{q}_{L, R} \Delta_{L, R}^{t} \cdot g_{i}\left(\Sigma / f_{\pi}\right) \cdot \Psi_{R, L}^{T, t}$ in (2.4), which will deliver no new structure to the low energy theory we will be considering further below. They would however be needed in general if both chiral fermions would mix with the same single composite field. Finally, the Lagrangian above can straightforwardly be generalized to the case of the SM fields mixing with more than one representation, each.

As we have explained, we do not consider the $b_{R}$, since it is expected to deliver a negligible contribution to the Higgs potential due to its small mixing with the composite sector. Note in that context that large changes in the diagonal Higgs couplings of the (light) fermions would generically also manifest themselves in large FCNCs [54]. Note also that, in all models considered besides those mixing the right-handed sector with a $\mathbf{1 0}$ of $\mathrm{SO}(5)$, the non-vanishing mass of the bottom quark would rely on an additional subleading mixing of $q_{L}$ with an appropriate multiplet $\Psi^{B}$ of the strong sector that features the correct $X$ charge to mix with the composite that couples with $b_{R}$. This term $\propto y_{L}^{b}$ has also been neglected, as it is again controlled by the rather small bottom mass - its smallness also helps to protect the $Z b_{L} b_{L}$ coupling which would receive unprotected corrections from this second mixing of $q_{L} \cdot{ }^{5}$ A similar discussion holds for the neutrino sector, which in the case of Dirac neutrinos is completely analogous. For Majorana neutrinos, $y_{R}^{\nu}$ could be non negligible, it's impact on the Higgs potential is however suppressed by a very large scale. The $\tau_{R}$ on the other hand is expected to exhibit a sizable composite component, as explained before, which can have an impact on the Higgs potential and thus we include the $\tau$ sector. $^{6}$

To be more explicit, we now specify explicitly the representation of $\mathrm{SO}(5)$ in which the composite fermions are embedded. In the following we will start with the fundamental representation $\mathbf{5}$ for concreteness, i.e., the $\mathrm{MCHM}_{5}$, however the discussion can easily be generalized to larger representations, which will be considered in the course of our study. Following the CCWZ approach $[34,35]$, we can write the Lagrangian $(2.4)$ in terms of representations of the unbroken global $\mathrm{SO}(4)$ symmetry, while keeping it $\mathrm{SO}(5)$ symmetric. To that extend we decompose the $\mathbf{5}$ of $\mathrm{SO}(5)$ into components $Q$ and $\tilde{T}$ that transform via $\hat{h}$ (see $(2.2)$ ) as a fourplet and a singlet under the unbroken $\mathrm{SO}(4)$ symmetry. Writing

$$
\begin{aligned}
& \Psi^{T}=U\left(Q^{T}, \tilde{T}^{T}\right)^{T}, \Psi^{t}=U\left(Q^{t}, \tilde{T}^{t}\right)^{T}, \\
& \Psi^{\mathcal{T}}=U\left(L^{\mathcal{T}}, \tilde{\mathcal{T}}^{\mathcal{T}}\right)^{T}, \Psi^{\tau}=U\left(L^{\tau}, \tilde{\mathcal{T}}^{\tau}\right)^{T},
\end{aligned}
$$

\footnotetext{
${ }^{5}$ Only in the $\mathbf{1 0}$ it is possible to mix both top and bottom sectors with composites with the same $\mathrm{U}(1)_{X}$ charge, due to the presence of several $\mathrm{SU}(2)_{L}$ singlets with different $T_{R}^{3}$, resulting in different $\mathrm{U}(1)_{Y}$ charges. This allows to generate masses for both $t$ and $b$ from a composite sector with a single $\mathrm{U}(1)_{X}$ charge, where the left handed mixing is parametrized by a common term $y_{L}$.

${ }^{6}$ Remember that in our approximation we also neglect the masses and Higgs couplings of the lighter SM fermions. Those terms would analogously be induced via mixings with their heavy composite partners at $m_{\Psi}$, however these are in general strongly suppressed with the small fermion masses and thus lead to a negligible contribution to the Higgs potential. The full 4D Lagrangian would include the heavy fermionic resonances sharing the flavor quantum numbers of the first two fermion generations. For a $4 \mathrm{D}$ setup including a comprehensive description of fermion and gauge resonances see e.g. [6, 17].
} 
we arrive at

$$
\begin{aligned}
\mathcal{L}_{\text {mass }}^{\mathrm{MCHM}_{5}{ }^{\prime}}= & -\sum_{f, f^{\prime}=T, t}\left(m_{f f^{\prime}} \bar{Q}_{L}^{f} Q_{R}^{f^{\prime}}+\tilde{m}_{f f^{\prime}} \overline{\tilde{T}}_{L}^{f} \tilde{T}_{R}^{f^{\prime}}\right)-y_{L}^{t} f_{\pi}\left(\bar{q}_{L} \Delta_{L}^{t}\right)_{I}\left(U_{I i} Q_{R}^{T^{i}}+U_{I 5} \tilde{T}_{R}^{T}\right) \\
& -y_{R}^{t} f_{\pi}\left(\bar{t}_{R} \Delta_{R}^{t}\right)_{I}\left(U_{I i} Q_{L}^{t}{ }^{i}+U_{I 5} \tilde{T}_{L}^{t}\right)+\text { h.c. } \\
& +(t \rightarrow \tau, T \rightarrow \mathcal{T}, q \rightarrow \ell, Q \rightarrow L),
\end{aligned}
$$

where $I=1, \ldots, 5, i=1, \ldots, 4$, and the composite-Yukawa parameters $Y_{1}^{f f^{\prime}}$ have been absorbed in the vector-like mass of the composite singlets $\tilde{m}_{f f^{\prime}} \equiv m_{\Psi}^{f f^{\prime}}+Y_{1}^{f f^{\prime}} f_{\pi}$, and $m_{f f^{\prime}}=m_{\Psi}^{f f^{\prime}}$.

We should note that while including both first layers of resonances, $\Psi^{T}$ and $\Psi^{t}$, at the first place was necessary to see how the $4 \mathrm{D}$ fields can be identified with different bulk fields of $\mathrm{AdS}_{5}$ (CFT) constructions of the composite Higgs idea, it is now convenient to remove the explicit appearance of one set of fields by going to a further effective low energy description, where now their effect is kept via new interactions. By integrating out $Q^{T}$ and $\tilde{T}^{t}$ we arrive at a theory that can in particular be matched directly to the $5 \mathrm{D}$ theory examined quantitatively in section 3 (to leading approximation). The setup then describes one-to-one just those modes that are most relevant for the generation of the Higgs potential and the SM-like fermion masses. ${ }^{7}$ In this way, all the fields that we keep correspond in particular to light custodians.

We thus finally arrive at the leading mass-mixing Lagrangian

$$
\begin{aligned}
\mathcal{L}_{\text {mass }}^{\mathrm{MCHM}_{5}=-\hat{m}_{T} \bar{Q}_{L} Q_{R}-\tilde{m}_{T} \overline{\tilde{T}}_{L} \tilde{T}_{R}-y_{L}^{t} f_{\pi}\left(\bar{q}_{L} \Delta_{L}^{t}\right)_{I}\left(a_{L}^{t} U_{I i} Q_{R}^{i}+b_{L}^{t} U_{I 5} \tilde{T}_{R}\right)} \\
\quad-y_{R}^{t} f_{\pi}\left(\bar{t}_{R} \Delta_{R}^{t}\right)_{I}\left(a_{R}^{t} U_{I i} Q_{L}^{i}+b_{R}^{t} U_{I 5} \tilde{T}_{L}\right)+\text { h.c. } \\
+(t \rightarrow \tau, T \rightarrow \mathcal{T}, q \rightarrow \ell, Q \rightarrow L)
\end{aligned}
$$

in agreement with [7], where we have removed the superscripts on $Q^{t}$ and $\tilde{T}^{T}$, while $\hat{m}_{T} \equiv$ $m_{t t}, \tilde{m}_{T} \equiv \tilde{m}_{T T}$, and $a_{L}^{t} \equiv m_{T t} / m_{T T} b_{L}^{t}$ arises from integrating out $Q^{T}$ at zero momentum, which leads to a linear interaction between $q_{L}$ and $Q_{R}$ via the term $\sim m_{T t}$ in (2.7). The $\mathcal{O}(1)$ coefficient $b_{L}^{t}\left(a_{R}^{t}\right)$ has been introduced for convenience [7] by rescaling $y_{L}^{t}\left(y_{R}^{t}\right)$. Analogously, from integrating out $\tilde{T}^{t}$ one obtains $b_{R}^{t} \equiv\left(\tilde{m}_{T t} / \tilde{m}_{t t}\right)^{*} a_{R}^{t}$. Actually, in this discussion we neglect subleading terms in ratios like $m_{f f^{\prime}} / m_{f f}$, assuming implicitly that the mass-mixings within the resonances are smaller than the diagonal mass terms. While this might be lifted in some regions of parameter space (like for large brane masses in section 3), the qualitative picture will not be changed by neglecting such terms here for simplicity. Note that in the following we will always work in a basis where the diagonal mass terms as well as the linear mixing parameters $y_{L, R}^{t}$ are real and positive.

While the Lagrangian (2.8) can be directly mapped to a 5D theory, it in particular also provides a simple and viable complete $4 \mathrm{D}$ model itself, employing just one composite vector-like resonance from the beginning [7, 17]. Adding the extra term $\sim g_{1}\left(\Sigma / f_{\pi}\right) \sim \Sigma \Sigma^{T}$,

\footnotetext{
${ }^{7}$ In the $5 \mathrm{D}$ theory the SM-like fields $q_{L}, t_{R}$ will not mix with the resonances corresponding to $Q^{T}$ and $\tilde{T}^{t}$ at leading order, see section 3 .
} 
mentioned above, to the linear mixing in (2.4) (as well as setting $T=t$ and removing the sum over the different composite excitations) one also obtains (2.8) without two distinct vector-like composite fields, however the connection to the $5 \mathrm{D}$ setup is then not transparent anymore. As mentioned before, we neglect the additional four similar terms with $t \rightarrow b, T \rightarrow B, Q \rightarrow Q^{b}$, which are generically negligible for the Higgs potential. The Lagrangian (2.8) will be the starting point for our analysis.

From invariance under $G_{\mathrm{EW}}$ one obtains for the fundamental representation of $\mathrm{SO}(5)$

$$
\begin{aligned}
\Delta_{L}^{t} & =\frac{1}{\sqrt{2}}\left(\begin{array}{ccccc}
0 & 0 & 1 & -i & 0 \\
1 & i & 0 & 0 & 0
\end{array}\right), \quad \Delta_{R}^{f}=-i\left(\begin{array}{lllll}
0 & 0 & 0 & 0 & 1
\end{array}\right) \\
\Delta_{L}^{\tau} & =\frac{1}{\sqrt{2}}\left(\begin{array}{ccccc}
1 & -i & 0 & 0 & 0 \\
0 & 0 & 1 & i & 0
\end{array}\right)
\end{aligned}
$$

$f=t, \tau$. The difference between the top-quark and $\tau$-lepton sectors arises since the corresponding SM-like doublets mix with different $T_{R}^{3}$ components of the $\mathrm{SU}(2)_{L} \times \mathrm{SU}(2)_{R}$ bi-doublets $Q^{T}, L^{T}$, respectively. This is dictated by the quantum numbers of the SM fermions, which fix the $\mathrm{U}(1)_{X}$ charges of the composites to $X_{t}=2 / 3$ and $X_{\tau}=-1$, for $\Psi^{T, t}$ and $\Psi^{\mathcal{T}, \tau}$. Note that the setup includes a protection for the $Z \bar{b}_{L} b_{L}$ and $Z \bar{\tau}_{R} \tau_{R}$ couplings via a custodial $P_{L R}$ symmetry exchanging $\mathrm{SU}(2)_{L} \leftrightarrow \mathrm{SU}(2)_{R}[37]$.

To zeroth order in $v / f$, i.e., neglecting EWSB, the Goldstone Matrix

$$
U \sim \mathbf{1}_{5 \times 5}+\mathcal{O}\left(v / f_{\pi}, \Pi\right)
$$

only mediates mixings of the SM doublet $q_{L}$ with $Q_{R}$ as well as of $t_{R}$ with $\tilde{T}_{L}$. Beyond that, the exact relative strength of the interactions of the SM fields with the fourplet and the singlet of $\mathrm{SO}(4)$ are not fixed by symmetry so far but rather parametrized by the ratios of $\mathcal{O}(1)$ numbers $a_{L, R}^{t} / b_{L, R}^{t}$. In the same approximation, one obtains simple analytic formulas for the masses of the heavy resonances after diagonalizing the fermionic mass matrix. Employing, with some abuse of notation, the same names for the mass eigenstates as for the interaction eigenstates (which coincide in the limit $y_{L, R} \rightarrow 0$ ) one obtains for the masses of the physical heavy resonances

$$
m_{T} \simeq \sqrt{\left(\hat{m}_{T}\right)^{2}+\left|a_{L}^{t}\right|^{2} y_{L}^{t 2} f_{\pi}^{2}}, \quad m_{\tilde{T}} \simeq \sqrt{\left(\tilde{m}_{T}\right)^{2}+\left|b_{R}^{t}\right|^{2} y_{R}^{t 2} f_{\pi}^{2}} .
$$

Expanding to the first non-trivial order in $v / f_{\pi}$ one arrives similarly at the top-quark mass

$$
m_{t}=\frac{\left|a_{L}^{t} a_{R}^{t} \tilde{m}_{T}-b_{L}^{t} b_{R}^{t} \hat{m}_{T}\right|}{2 \sqrt{2}} \sin \varphi_{L}^{t} \sin \varphi_{R}^{t} \sin \left(2 v / f_{\pi}\right)
$$

where the rotation angles

$$
\sin \varphi_{L}^{t}=\frac{y_{L}^{t} f_{\pi}}{m_{T}}, \quad \sin \varphi_{R}^{t}=\frac{y_{R}^{t} f_{\pi}}{m_{\tilde{T}}}
$$

describe the admixture of the composite modes into the light SM-like top quark. Inspecting these equations, one can thus see explicitly the mechanism of partial compositeness at 
work - the mass of a SM-like fermion is proportional to its mixings $\sin \varphi_{L, R}^{t}$ with the strong sector. Since after EWSB this mixing involves fields with different quantum numbers under $G_{\mathrm{SM}}$, it is a source of tree-level FCNCs via non-universal gauge interactions in the flavor basis. This mechanism nicely matches with the experimental observation that FCNCs are more constrained for the light generations. Note that the same discussion as above holds for leptons with the aforementioned replacements of indices. Finally, employing $a_{L}^{t} \sim a_{R}^{t} \sim b_{L}^{t} \sim b_{R}^{t} \sim 1$, we obtain to leading approximation

$$
m_{t} \sim \frac{y_{L}^{t} y_{R}^{t} f_{\pi}}{\sqrt{2} \min \left(m_{T}, m_{\tilde{T}}\right)} v
$$

\subsection{The Higgs potential and light partners}

While the Higgs potential $V(\Pi)$ is zero at tree level, it receives non-vanishing contributions at one loop from the explicit breaking of $\mathrm{SO}(5)$, as discussed before. Since the gauge boson contributions are subleading (being proportional to the weak gauge coupling) with no qualitative effect on the mechanism of EWSB and in particular do not contribute significantly to the Higgs mass, we will neglect them for the discussion of this section. We will however take into account their full numerical impact, including the heavy resonances, in section 3.

Making use of symmetry arguments, we can already deduce the form of the potential directly from the mass-mixing Lagrangian, i.e., (2.8) for the $\mathrm{MCHM}_{5}$. To that extent, we restore the approximate $\mathrm{SO}(5)$ global symmetry formally by promoting the coupling matrices $\Delta_{L, R}^{f}$ to spurions $\hat{\Delta}_{L, R}^{f}$, transforming under the global SO(5) of the strong sector in the same way the corresponding resonances do. If the $\hat{\Delta}_{L, R}^{f}$ transform in addition appropriately under the elementary symmetry group $\mathrm{SU}(2)_{L}^{0} \times \mathrm{U}(1)_{R}^{0}$ of the $q, t, l, \tau$ fermions, also the linear mixings are invariant under the full global symmetry of the rest of the Lagrangian. As a consequence, also the Higgs potential needs to formally respect the $\mathrm{SO}(5)$ symmetry (and the elementary symmetry), which should then be broken by the vevs of the spurions $\left\langle\hat{\Delta}_{L, R}^{f}\right\rangle=\Delta_{L, R}^{f}$ in order to generate a non-trivial potential. Thus the form of the Higgs potential can be constructed by forming all possible invariants under the full global symmetry, containing at least one spurion $\Delta_{L, R}^{f}$, set to its vev, and the Goldstone-Higgs matrix $U$. As the spurions are always accompanied by the linear mixing parameters $y_{L, R}^{f}$, taking the role of an expansion parameter, a series in powers of the spurions can be established. In order for the elementary $\mathrm{SU}(2)_{L}^{0} \times \mathrm{U}(1)_{R}^{0}$ symmetry to be respected, the spurions can only enter in the combinations $\Delta_{L}^{f^{\dagger}} \Delta_{L}^{f}$ and $\Delta_{R}^{f^{\dagger}} \Delta_{R}^{f}$. In the following we will first focus on the quark sector, whereas the impact of leptons will be analyzed in the next subsection.

$\mathbf{M C H M}_{\mathbf{5}}$. For the discussed case of $q_{L}, t_{R}$ both mixing with a 5 of $\mathrm{SO}(5)$, the form of the potential at $\mathcal{O}\left(\Delta^{2}\right)$ is thus fixed to

$$
V_{2}^{(5)}(h)=\frac{N_{c} m_{\Psi}^{4}}{16 \pi^{2}}\left[\frac{y_{L}^{t 2}}{g_{\Psi}^{2}} c_{L}^{t} v_{L}^{(5)}(h)+\frac{y_{R}^{t 2}}{g_{\Psi}^{2}} c_{R}^{t} v_{R}^{(5)}(h)\right],
$$

where the prefactors follow from naive dimensional analysis and the fact that the considered fermions enter in $N_{c}=3$ colors. The concrete values for the coefficients $c_{L, R}$, which are 
generically of $\mathcal{O}(1)$, need to be determined from an explicit calculation and cannot be fixed by symmetries alone. Nevertheless, the $\mathrm{SO}(5)$ symmetry already tells us that the Higgs field can only enter in two structures at this order

$$
\begin{aligned}
& v_{L}^{(5)}(h)=\left(U^{T} \Delta_{L}^{t}{ }^{\dagger} \Delta_{L}^{t} U\right)_{55}=\frac{1}{2} \sin ^{2}\left(h / f_{\pi}\right), \\
& v_{R}^{(5)}(h)=\left(U^{T} \Delta_{R}^{t}{ }^{\dagger} \Delta_{R}^{t} U\right)_{55}=\cos ^{2}\left(h / f_{\pi}\right)=1-\sin ^{2}\left(h / f_{\pi}\right),
\end{aligned}
$$

where we have employed (2.9) and the explicit form of the Goldstone matrix as given in appendix A. We inspect that, since the constant term in the second line can be neglected in the Higgs potential, only one functional dependence on the Higgs field is present. The combinations of spurions exhibit a block-diagonal structure and do not mix the fifth component of $U_{I 5}=\Sigma / f_{\pi}$ with the other four components. In consequence (dropping a constant), we get

$$
V_{2}^{(5)}(h) \cong \frac{N_{c} m_{\Psi}^{4}}{16 \pi^{2} g_{\psi}^{2}}\left[c_{L}^{t} \frac{y_{L}^{t 2}}{2}-c_{R}^{t} y_{R}^{t 2}\right] \sin ^{2}\left(h / f_{\pi}\right) .
$$

This leading contribution to the potential does however not yet lead to a viable phenomenology. Its minimum is realized for $h=0, f_{\pi} \frac{\pi}{2}, \ldots$, which means that we can not have a realistic symmetry breaking with $0<v<f_{\pi}$. To fix this problem we need to take into account formally subleading contributions $[6,7,9]$. While no new independent $\mathrm{SO}(5)$ invariant structures appear at $\mathcal{O}\left(\Delta^{4}\right)$, one can have products of the structures (2.16) which lead to a different trigonometric dependence on $h$,

$$
\begin{aligned}
V_{4}^{(5)}(h)=\frac{N_{c} m_{\Psi}^{4}}{16 \pi^{2}}\left[\frac{y_{L}^{t 4}}{g_{\Psi}^{4}} c_{L L}^{t}\left[v_{L}^{(5)}(h)\right]^{2}+\frac{y_{R}^{t 4}}{g_{\Psi}^{4}} c_{R R}^{t}\left[v_{R}^{(5)}(h)\right]^{2}+\frac{y_{L}^{t 2} y_{R}^{t 2}}{g_{\Psi}^{4}} c_{L R}^{t} v_{L}^{(5)}(h) v_{R}^{(5)}(h)\right] \\
\cong \frac{N_{c} m_{\Psi}^{4}}{16 \pi^{2} g_{\Psi}^{4}}\left[\left(c_{L L}^{t} \frac{y_{L}^{t 4}}{4}-c_{R R}^{t} y_{R}^{t 4}\right) \sin ^{2}\left(h / f_{\pi}\right)\right. \\
\left.-\left(c_{L L}^{t} \frac{y_{L}^{t 4}}{4}+c_{R R}^{t} y_{R}^{t 4}-c_{L R}^{t} \frac{y_{L}^{t 2} y_{R}^{t 2}}{2}\right) \sin ^{2}\left(h / f_{\pi}\right) \cos ^{2}\left(h / f_{\pi}\right)\right] .
\end{aligned}
$$

However, since usually the elementary-composite mixings are significantly smaller than the strong coupling (see (2.12)), $y_{L, R}^{t} \ll g_{\Psi}$, the fact that $V_{4}^{(5)}(h)$ needs to have a comparable impact to $V_{2}^{(5)}(h)$ worsens the tuning of the model. ${ }^{8}$

In particular, defining generally

$$
V(h)=V_{2}(h)+V_{4}(h) \equiv \alpha \sin ^{2}\left(h / f_{\pi}\right)-\beta \sin ^{2}\left(h / f_{\pi}\right) \cos ^{2}\left(h / f_{\pi}\right),
$$

we naturally obtain $\alpha \sim y_{L, R}^{t 2} / g_{\Psi}^{2}$ and $\beta \sim y_{L, R}^{t 2} y_{L, R}^{t 2} / g_{\Psi}^{4}$ in the $\mathrm{MCHM}_{5}$. In order to allow for a viable EWSB, the leading contribution to $\alpha$, originating from $V_{2}^{(5)}(h)$, needs to feature a tuning within its contributions that brings it from its natural size of $\mathcal{O}\left(y_{L, R}^{t 2} / g_{\Psi}^{2}\right)$ down to $\mathcal{O}\left(y_{L, R}^{t 4} / g_{\Psi}^{4}\right) .{ }^{9}$ Explicitly, the (non-trivial) minimum of the potential (2.19) occurs at

$$
\sin ^{2}\left(h / f_{\pi}\right)=\frac{\beta-\alpha}{2 \beta},
$$

\footnotetext{
${ }^{8}$ Note that we indeed consider a strongly coupled new physics sector, $g_{\Psi} \gg 1$. Leaving this class of models, the considerations regarding the tuning could be altered.

${ }^{9}$ From (2.17) we see that for the $\mathrm{MCHM}_{5}$ realistic EWSB thus requires $c_{L}^{t} y_{L}^{t 2}=2 c_{R}^{t} y_{R}^{t 2}\left(1+\mathcal{O}\left(\frac{y_{L, R}^{2}}{g_{\Psi}^{2}}\right)\right)$. In consequence we will denote $y_{t} \equiv y_{R}^{t} \sim y_{L}^{t}$.
} 
which requires $\alpha-\beta$ to be as small as

$$
\alpha-\beta=-2 \beta \sin ^{2}\left(v / f_{\pi}\right)
$$

in order to allow for the sought solution. Comparing this required size to its natural size of $\alpha-\beta \sim y_{t}^{2} / g_{\Psi}^{2}$, we obtain the famous "double tuning" [7]

$$
\Delta_{(5)}^{-1} \sim \frac{y_{t}^{4} / g_{\Psi}^{4} \sin ^{2}\left(v / f_{\pi}\right)}{y_{t}^{2} / g_{\Psi}^{2}}=\frac{y_{t}^{2}}{g_{\Psi}^{2}} \sin ^{2}\left(v / f_{\pi}\right)
$$

i.e., the coefficients entering $V(h)$ need not only to cancel to $\sim \sin ^{2}\left(v / f_{\pi}\right) y_{t}^{2} / g_{\Psi}^{2} \ll y_{t}^{2} / g_{\Psi}^{2}$ (the standard tuning due to $v \ll f$ ), but in the $\mathrm{MCHM}_{5}$ another tuning in the contributions to $V_{2}$ is required to make it also one order smaller in $y_{t}^{2} / g_{\Psi}^{2}$.

Moreover we observe that

$$
\begin{aligned}
& m_{H}^{2}=\frac{8}{f_{\pi}^{2}} \cos ^{2}\left(v / f_{\pi}\right) \sin ^{2}\left(v / f_{\pi}\right) \beta \\
& \stackrel{\mathrm{MCHM}_{5}}{=} f_{\pi}^{2} \frac{N_{c}}{2 \pi^{2}}\left(\frac{c_{L L}^{t}}{4} y_{L}^{t 4}-\frac{c_{L R}^{t}}{2} y_{L}^{t 2} y_{R}^{t 2}+c_{R R}^{t} y_{R}^{t 4}\right) \cos ^{2}\left(v / f_{\pi}\right) \sin ^{2}\left(v / f_{\pi}\right),
\end{aligned}
$$

and thus

$$
m_{H}^{(5)} \sim \sqrt{\frac{3}{2 \pi^{2}}} y_{t}^{2} v
$$

Comparing this with (2.14), one obtains the relation [7]

$$
m_{H}^{(5)} \sim \frac{\sqrt{3}}{\pi} \frac{\min \left(m_{T}, m_{\tilde{T}}\right)}{f_{\pi}} m_{t} .
$$

From this we can see explicitly that generically $m_{H}^{(5)} \gtrsim m_{t}$. Finally, we also can read off directly that a light Higgs boson, as found at the LHC, requires light partners in the $\mathrm{MCHM}_{5}, \min \left(m_{T}, m_{\tilde{T}}\right) \ll m_{\Psi}$. For example for $f_{\pi}=500 \mathrm{GeV}$ as well as $m_{H} \lesssim 110 \mathrm{GeV}$ (see section 3.2.1) and $m_{t} \sim 150 \mathrm{GeV}$ we obtain

$$
\min \left(m_{T}, m_{\tilde{T}}\right) \lesssim 650 \mathrm{GeV}
$$

which is already in slight conflict with LHC searches [55]. In contrast, the general scale of fermionic resonances would only be at $m_{\Psi} \sim 2 \mathrm{TeV}$ (assuming $g_{\Psi} \sim 4$ ). For $f_{\pi}=800 \mathrm{GeV}$ this becomes still only $\min \left(m_{T}, m_{\tilde{T}}\right) \lesssim 1 \mathrm{TeV}$, while the rest of the resonances clearly leave the LHC reach, $m_{\Psi}>3 \mathrm{TeV}$. These findings will be confirmed for explicit $5 \mathrm{D}$ realizations of the composite Higgs framework in section 3. Raising $f_{\pi}$ even further is problematic in the context of addressing the fine tuning problem, since the tuning increases quadratically with $f_{\pi}$.

In the following we will first review how the requirement for light partners and/or the double tuning could be changed by enlarging the embedding of the quark sector of the composite models. We will then show that, compared to modifications of the quark realizations, taking into account the effects from realistic embeddings of the lepton sector 
allows to significantly raise the masses of the top partners in a more natural way, i.e., avoiding a significant ad-hoc increase in the complexity of the model with respect to minimal realizations. We will also see that considering the lepton sector opens the possibility to avoid the ad-hoc tuning which generically emerges by modifying the quark embeddings in a way that avoids light partners.

$\mathrm{MCHM}_{10}$. Let us start by mixing the fermions with 10 s of $\mathrm{SO}(5)$, i.e., the $\mathrm{MCHM}_{10}$. The decomposition into representations of $\mathrm{SO}(4) \cong \mathrm{SU}(2)_{L} \times \mathrm{SU}(2)_{R}$ now follows $\mathbf{1 0}=$ $(\mathbf{3}, \mathbf{1}) \oplus(\mathbf{1}, \mathbf{3}) \oplus(\mathbf{2}, \mathbf{2})$ and reads explicitly

$$
\begin{aligned}
& \Psi^{T}=U\left[\left(T_{(3,1)}^{T}, T_{(1,3)}^{T}, Q^{T}\right)^{A} T^{A}\right] U^{T}, \quad \Psi^{t}=U\left[\left(T_{(3,1)}^{t}, T_{(1,3)}^{t}, Q^{t}\right)^{A} T^{A}\right] U^{T}, \\
& \Psi^{\mathcal{T}}=U\left[\left(T_{(3,1)}^{\mathcal{T}}, T_{(1,3)}^{\mathcal{T}}, Q^{\mathcal{T}}\right)^{A} T^{A}\right] U^{T}, \quad \Psi^{\tau}=U\left[\left(T_{(3,1)}^{\tau}, T_{(1,3)}^{\tau}, Q^{\tau}\right)^{A} T^{A}\right] U^{T},
\end{aligned}
$$

where the $\mathrm{SU}(2)_{L}\left(\mathrm{SU}(2)_{R}\right)$ triplet is represented by a three-component vector $T_{(3,1)}\left(T_{(1,3)}\right)$, while the bi-doublet $Q$ has four entries, such that $A=1, \ldots, 10$, and the generators $T^{A}$ are defined in appendix A. The abelian charges read again $X_{t}=2 / 3$ and $X_{\tau}=-1$, for $\Psi^{T, t}$ and $\Psi^{\mathcal{T}, \tau}$, in order to protect the $Z \bar{b}_{L} b_{L}$ and $Z \bar{\tau}_{R} \tau_{R}$ vertices. In complete analogy to as discussed for the $\mathrm{MCHM}_{5}$, the Higgs potential can be studied to leading approximation by considering an effective theory containing only the composite resonances $Q^{t}, T_{(1,3)}^{T}, T_{(3,1)}^{T}$, while the other modes will be integrated out. ${ }^{10}$

Thus, in analogy to (2.8), we finally obtain the mass-mixing Lagrangian

$$
\begin{aligned}
\mathcal{L}_{\text {mass }}^{\mathrm{MCHM}_{10}=} & -\hat{m}_{T} \bar{Q}_{L} Q_{R}-\tilde{m}_{T}\left(\bar{T}_{(3,1) L} T_{(3,1) R}+\bar{T}_{(1,3) L} T_{(1,3) R}\right) \\
& -y_{L}^{t} f_{\pi} \operatorname{Tr}\left\{\left(\bar{q}_{L} \boldsymbol{\Delta}_{L}^{t}\right) U\left(a_{L}^{t} Q_{R}^{\hat{a}} T^{\hat{a}}+b_{L}^{t} T_{(1,3)}^{a} T_{R}^{a}+b_{L}^{t} T_{(3,1)}^{a} T_{L} T_{L}^{a}\right) U^{T}\right\} \\
& -y_{R}^{t} f_{\pi} \operatorname{Tr}\left\{\left(\bar{t}_{R} \boldsymbol{\Delta}_{R}^{t}\right) U\left(a_{R}^{t} Q_{L}^{\hat{a}} T^{\hat{a}}+b_{R}^{t} T_{(1,3)}^{a} T_{R}^{a}+b_{R}^{t} T_{(3,1)}^{a} T_{L}^{a}\right) U^{T}\right\}+\text { h.c. } \\
& +(t \rightarrow \tau, T \rightarrow \mathcal{T}, q \rightarrow \ell, Q \rightarrow L),
\end{aligned}
$$

where we removed the superscripts on $Q^{t}, T_{(1,3)}^{T}, T_{(3,1)}^{T}$, while $\hat{m}_{T} \equiv m_{\Psi}^{t t}+Y_{1}^{t t} / 2, \tilde{m}_{T} \equiv m_{\Psi}^{T T}$, and $\hat{a}=1, \ldots, 4, a=1, \ldots, 3$. The coefficients $a_{L}^{t} \equiv\left(m_{\Psi}^{T t}+Y_{1}^{T t} / 2\right) /\left(m_{\Psi}^{T T}+Y_{1}^{T T} / 2\right) b_{L}^{t}$ and $b_{R}^{t} \equiv\left(m_{\Psi}^{T t} / m_{\Psi}^{t t}\right)^{*} a_{R}^{t}$ now arise from integrating out $Q^{T}, T_{(1,3)}^{t}, T_{(3,1)}^{t}$ at zero momentum, where $b_{L}^{t}\left(a_{R}^{t}\right)$ has again been introduced for convenience by rescaling $y_{L}^{t}\left(y_{R}^{t}\right)$.

The spurions that restore the $\mathrm{SO}(5)$ symmetry now also transform in adjoint representations and take the vevs

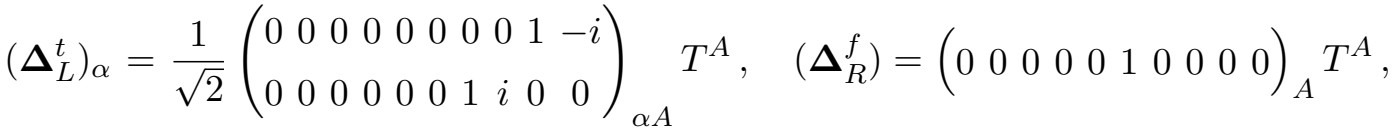

$$
\begin{aligned}
& \left(\boldsymbol{\Delta}_{L}^{\tau}\right)_{\alpha}=\frac{1}{\sqrt{2}}\left(\begin{array}{llllllllll}
0 & 0 & 0 & 0 & 0 & 0 & 1 & -i & 0 & 0 \\
0 & 0 & 0 & 0 & 0 & 0 & 0 & 0 & 1 & i
\end{array}\right)_{\alpha A},
\end{aligned}
$$

where $\alpha=1,2$ are $\mathrm{SU}(2)_{L}$ indices.

\footnotetext{
${ }^{10}$ Again, they do not couple at leading order to the light SM-like modes in the 5D picture and the resulting setup corresponds to a viable complete $4 \mathrm{D}$ model involving a single 10 of $\mathrm{SO}(5)$.
} 
In complete analogy to the case of the $\mathrm{MCHM}_{5}$, the spurion analysis leads to the quark contribution

$$
V_{2}^{(10)}(h)=\frac{N_{c} m_{\Psi}^{4}}{16 \pi^{2}}\left[\frac{y_{L}^{q 2}}{g_{\Psi}^{2}} c_{L}^{q} v_{L}^{(10)}(h)+\frac{y_{R}^{t 2}}{g_{\Psi}^{2}} c_{R}^{t} v_{R}^{(10)}(h)\right],
$$

where now

$$
\begin{aligned}
& v_{L}^{(10)}(h)=\left(U^{T}\left(\boldsymbol{\Delta}_{L}^{q \dagger}\right)^{\alpha}\left(\boldsymbol{\Delta}_{L}^{q}\right)_{\alpha} U\right)_{55}=\frac{1}{2}-\frac{3}{8} \sin ^{2}\left(h / f_{\pi}\right), \\
& v_{R}^{(10)}(h)=\left(U^{T} \boldsymbol{\Delta}_{R}^{t}{ }^{\dagger} \boldsymbol{\Delta}_{R}^{t} U\right)_{55}=\frac{1}{4} \sin ^{2}\left(h / f_{\pi}\right) .
\end{aligned}
$$

With some abuse of notation we do not introduce new names for the other parameters, which can deviate from the case of the $\mathrm{MCHM}_{5}$. As in the $\mathrm{MCHM}_{5}$, due to the blockdiagonal form of both combinations of spurions, there is again only one trigonometric dependence on the Higgs field at second order in the spurions. We obtain

$$
V_{2}^{(10)}(h) \cong \frac{N_{c} m_{\Psi}^{4}}{16 \pi^{2} g_{\psi}^{2}}\left[-\frac{3}{8} c_{L}^{q} y_{L}^{q 2}+\frac{1}{4} c_{R}^{t} y_{R}^{t 2}\right] \sin ^{2}\left(h / f_{\pi}\right)
$$

Once more, formally subleading contributions to the potential are essential for a viable electroweak symmetry breaking, while the above terms need to cancel at $\mathcal{O}\left(\frac{y_{L, R}^{2}}{g_{\Psi}^{2}}\right) .{ }^{11}$

At $\mathcal{O}\left(\boldsymbol{\Delta}^{4}\right)$, new non-trivial $\mathrm{SO}(5)$ invariant structures

$$
\begin{aligned}
v_{L L(k)}^{(10)} & \sim\left(\left(\boldsymbol{\Delta}_{L}\right)_{i j}^{\alpha}\right)^{4}\left(U_{m 5}\right)^{2,4} \\
v_{L R(k)}^{(10)} & \sim\left(\left(\boldsymbol{\Delta}_{L}\right)_{i j}^{\alpha}\right)^{2}\left(\left(\boldsymbol{\Delta}_{R}\right)_{k l}\right)^{2}\left(U_{m 5}\right)^{2,4} \\
v_{R R(k)}^{(10)} & \sim\left(\left(\boldsymbol{\Delta}_{R}\right)_{i j}\right)^{4}\left(U_{m 5}\right)^{2,4}
\end{aligned}
$$

can be formed in the $\mathrm{MCHM}_{10}$, by contracting the matrix-indices of the spurions and Goldstone matrices in various different ways. ${ }^{12}$ In the end however, as for the $\mathrm{MCHM}_{5}$, only the trigonometric functions $\sin ^{2}\left(h / f_{\pi}\right)$ and $\sin ^{2}\left(h / f_{\pi}\right) \cos ^{2}\left(h / f_{\pi}\right)$ emerge,

$$
\begin{aligned}
& V_{4}^{(10)}(h)= \frac{N_{c} m_{\Psi}^{4}}{16 \pi^{2}} \sum_{k}\left[\frac{y_{L}^{q 4}}{g_{\Psi}^{4}} c_{L L}^{q(k)} v_{L L(k)}^{(10)}+\frac{y_{R}^{t 4}}{g_{\Psi}^{4}} c_{R R}^{t(k)} v_{R R(k)}^{(10)}+\frac{y_{L}^{q 2} y_{R}^{t 2}}{g_{\Psi}^{4}} c_{L R}^{q(k)} v_{L R(k)}^{(10)}\right] \\
& \cong \frac{N_{c} m_{\Psi}^{4}}{16 \pi^{2} g_{\Psi}^{4}}\left[\left(c_{L L}^{q} y_{L}^{q 4}+c_{R R}^{t} y_{R}^{t 4}+c_{L R}^{q} y_{L}^{q 2} y_{R}^{t 2}\right) \sin ^{2}\left(h / f_{\pi}\right)\right. \\
&\left.+\left(c_{L L}^{q q} y_{L}^{q 4}+c_{R R}^{\prime t} y_{R}^{t 4}+c_{L R}^{q q} y_{L}^{q 2} y_{R}^{t 2}\right) \sin ^{2}\left(h / f_{\pi}\right) \cos ^{2}\left(h / f_{\pi}\right)\right],
\end{aligned}
$$

where $c_{L L, R R, L R}^{(\prime) f}$ are generically $\mathcal{O}(1)$ coefficients emerging as linear combinations of the coefficients $c_{L L, R R, L R}^{f(k)}$ of the different $\mathrm{SO}(5)$ invariant structures $v_{L L, R R, L R(k)}^{(10)}$. As a consequence of the above discussion, the tuning is clearly of the same order as in the $\mathrm{MCHM}_{5}$

$$
\Delta_{(10)}^{-1} \sim \Delta_{(5)}^{-1} \sim \frac{y_{t}^{2}}{g_{\Psi}^{2}} \sin ^{2}\left(v / f_{\pi}\right),
$$

and again the small Higgs mass suggests light partners.

\footnotetext{
${ }^{11}$ Specifically, one needs $\frac{3}{2} c_{L}^{q} y_{L}^{q 2}=c_{R}^{t} y_{R}^{t 2}\left(1+\mathcal{O}\left(\frac{y_{L, R}^{2}}{g_{\Psi}^{2}}\right)\right)$ and thus again $y_{R}^{t} \sim y_{L}^{q}$.

${ }^{12}$ Note that in $\mathrm{SO}(5)$ invariant combinations the Goldstone matrices only enter as $U_{r 5}$ and vanish to the identity as $U^{T} U=\mathbf{1}$ in other possible contractions of indices.
} 
$\mathbf{M C H M}_{\mathbf{1 0 - 5}}, \mathbf{M C H M}_{\mathbf{5 - 1 0}}$. Mixing the left chirality with a $\mathbf{1 0}$ and the right with a $\mathbf{5}$ (denoted by the first and second subscript on MCHM, respectively), like $\Psi^{T} \sim \mathbf{1 0}, \Psi^{t} \sim \mathbf{5}$ in (2.4), or vice versa, does not change the picture qualitatively. It just corresponds to replacing only one of the $v_{A}^{(5)}$ in (2.15) with a $v_{A}^{(10)}, A=L, R$. Since we found that both feature the same trigonometric dependence, this leads to the same conclusions, up to $\mathcal{O}(1)$ factors. Moreover, it is evident from the discussion above that one can not mix the $t_{R}$ with a singlet of $\mathrm{SO}(5)$ only - not contributing to $V(h)$ - if one wants to achieve a realistic EWSB, given that the left handed top mixes with either a $\mathbf{5}$ or a $\mathbf{1 0}$. We conclude that at least one chirality needs to mix with a more complex composite fermion realization than a single fundamental or adjoint if we want to see qualitatively new features in the quark sector.

$\mathbf{M C H M}_{\mathbf{1 4}}$. If we embed the fermions in the symmetric representation, a $\mathbf{1 4}$ of $\mathrm{SO}(5)$, new features emerge. We start with assigning both SM chiralities to a $\mathbf{1 4}$ of the strong sector, however as we will detail below, the generic features will remain if only one of them mixes with a symmetric representation.

The decomposition into $\mathrm{SU}(2)_{L} \times \mathrm{SU}(2)_{R} \quad$ representations via $\mathbf{1 4}=(\mathbf{3}, \mathbf{3}) \oplus(\mathbf{2}, \mathbf{2}) \oplus(\mathbf{1}, \mathbf{1})$ now reads

$$
\begin{array}{ll}
\Psi^{T}=U\left[\left(T_{(3,3)}^{T}, Q^{T}, \tilde{T}^{T}\right)^{A} \hat{T}^{A}\right] U^{T}, & \Psi^{t}=U\left[\left(T_{(3,3)}^{t}, Q^{t}, \tilde{T}^{t}\right)^{A} \hat{T}^{A}\right] U^{T}, \\
\Psi^{\mathcal{T}}=U\left[\left(T_{(3,3)}^{\mathcal{T}}, Q^{\mathcal{T}}, \tilde{T}^{\mathcal{T}}\right)^{A} \hat{T}^{A}\right] U^{T}, & \Psi^{\tau}=U\left[\left(T_{(3,3)}^{\tau}, Q^{\tau}, \tilde{T}^{\tau}\right)^{A} \hat{T}^{A}\right] U^{T},
\end{array}
$$

where the $\mathrm{SU}(2)_{L} \times \mathrm{SU}(2)_{R}$ bi-triplet is represented by the nine-component vector $T_{(3,3)}$, while the bi-doublet is again denoted by $Q$ and the singlet by $\tilde{T}$, such that now $A=$ $1, \ldots, 14$, see appendix A for the definition of $\hat{T}^{A}$. The abelian charges read once more $X_{t}=2 / 3$ and $X_{\tau}=-1$, for $\Psi^{T, t}$ and $\Psi^{\mathcal{T}, \tau}$, respectively, protecting still $Z \bar{b}_{L} b_{L}$ and $Z \bar{\tau}_{R} \tau_{R}$. Again, the Higgs potential can be studied conveniently to leading approximation after integrating out $Q^{T}, \tilde{T}^{t}, T_{(3,3)}^{t}$, leading again to a viable $4 \mathrm{D}$ model of effectively only one composite resonance. ${ }^{13}$

Thus, again in analogy to (2.8), we obtain the final mass-mixing Lagrangian

$$
\begin{aligned}
\mathcal{L}_{\text {mass }}^{\mathrm{MCHM}_{14}}= & -\hat{m}_{T} \bar{Q}_{L} Q_{R}-\tilde{m}_{T} \overline{\tilde{T}}_{L} \tilde{T}_{R}-m_{(3,3)} \bar{T}_{(3,3) L} T_{(3,3) R} \\
& -y_{L}^{t} f_{\pi} \operatorname{Tr}\left\{\left(\bar{q}_{L} \boldsymbol{\Delta}_{L}^{t}\right) U\left(a_{L}^{t} Q_{R}^{\hat{a}} \hat{T}^{\hat{a}+9}+b_{L}^{t} \tilde{T}_{R} \hat{T}^{14}+b_{L}^{t} T_{(3,3)}^{\beta} \hat{T}^{\beta}\right) U^{T}\right\} \\
& -y_{R}^{t} f_{\pi} \operatorname{Tr}\left\{\left(\bar{t}_{R} \boldsymbol{\Delta}_{R}^{t}\right) U\left(a_{R}^{t} Q_{L}^{\hat{a}} \hat{T}^{\hat{a}+9}+b_{R}^{t} \tilde{T}_{L} \hat{T}^{14}+c_{R}^{t} T_{(3,3)_{L}}^{\beta} \hat{T}^{\beta}\right) U^{T}\right\}+\text { h.c. } \\
& +(t \rightarrow \tau, T \rightarrow \mathcal{T}, q \rightarrow \ell, Q \rightarrow L),
\end{aligned}
$$

where we removed the superscripts on $Q^{t}, \tilde{T}^{T}, T_{(3,3)}^{T}$, while $\hat{m}_{T} \equiv m_{\Psi}^{t t}+Y_{1}^{t t} / 2, \tilde{m}_{T} \equiv$ $m_{\Psi}^{T T}+4 Y_{1}^{T T} / 5+4 Y_{2}^{T T} / 5, m_{(3,3)} \equiv m_{\Psi}^{T T}$, and $\hat{a}=1, \ldots, 4, \beta=1, \ldots, 9$. The coefficients $a_{L}^{t} \equiv\left(m_{\Psi}^{T t}+Y_{1}^{T t} / 2\right) /\left(m_{\Psi}^{T T}+Y_{1}^{T T} / 2\right) b_{L}^{t}, b_{R}^{t} \equiv\left(\left(m_{\Psi}^{T t}+4 Y_{1}^{T t} / 5+4 Y_{2}^{T t} / 5\right) /\left(m_{\Psi}^{t t}+4 Y_{1}^{t t} / 5+\right.\right.$ $\left.\left.4 Y_{2}^{t t} / 5\right)\right)^{*} a_{R}^{t}$ and $c_{R}^{t} \equiv\left(m_{\Psi}^{T t} / m_{\Psi}^{t t}\right)^{*} a_{R}^{t}$ now arise from integrating out $Q^{T}, \tilde{T}^{t}, T_{(3,3)}^{t}$ at zero momentum, where $b_{L}^{t}\left(a_{R}^{t}\right)$ has again been introduced for convenience by rescaling $y_{L}^{t}\left(y_{R}^{t}\right)$.

\footnotetext{
${ }^{13}$ Also in the 14, these modes do not couple at leading order to the light SM-like fields in the 5D picture.
} 
The spurions that restore the $\mathrm{SO}(5)$ symmetry now transform in symmetric representations of $\mathrm{SO}(5)$ and acquire the vevs

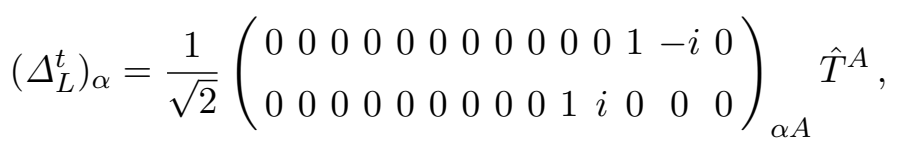

$$
\begin{aligned}
& \left(\Delta_{L}^{\tau}\right)_{\alpha}=\frac{1}{\sqrt{2}}\left(\begin{array}{cccccccccccccc}
0 & 0 & 0 & 0 & 0 & 0 & 0 & 0 & 0 & 1 & -i & 0 & 0 & 0 \\
0 & 0 & 0 & 0 & 0 & 0 & 0 & 0 & 0 & 0 & 0 & 1 & i & 0
\end{array}\right)_{\alpha A} \hat{T}^{A},
\end{aligned}
$$

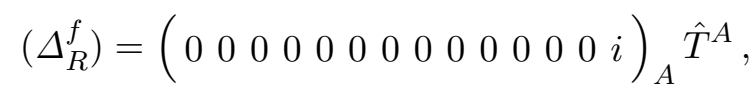

where $\alpha=1,2$ are $\mathrm{SU}(2)_{L}$ indices.

In contrast to the $\mathrm{MCHM}_{5}$ and $\mathrm{MCHM}_{10}$, the spurion analysis shows that now already at $\mathcal{O}\left(\Delta^{2}\right)$ two different trigonometric structures arise, which has interesting consequences for the quark [10] and in particular the lepton sector. Focusing first on the former, we find

$$
V_{2}^{(14)}(h)=\frac{N_{c} m_{\Psi}^{4}}{16 \pi^{2}}\left[\frac{y_{L}^{t 2}}{g_{\Psi}^{2}}\left(c_{L}^{t(1)} v_{L(1)}^{(14)}+c_{L}^{t(2)} v_{L(2)}^{(14)}\right)+\frac{y_{R}^{t 2}}{g_{\Psi}^{2}}\left(c_{R}^{t(1)} v_{R(1)}^{(14)}+c_{R}^{t(2)} v_{R(2)}^{(14)}\right)\right]
$$

where now

$$
\begin{aligned}
& v_{L(1)}^{(14)}=\left(U^{T}\left(\Delta_{L}^{t}\right)^{\alpha}\left(\Delta_{L}^{t}\right)_{\alpha} U\right)_{55}=1-\frac{3}{4} \sin ^{2}\left(h / f_{\pi}\right) \\
& v_{L(2)}^{(14)}=\left(U^{T}\left(\Delta_{L}^{t^{\dagger}}\right)^{\alpha} U\right)_{55}\left(U^{T}\left(\Delta_{L}^{t}\right)_{\alpha} U\right)_{55}=\cos ^{2}\left(h / f_{\pi}\right) \sin ^{2}\left(h / f_{\pi}\right) \\
& v_{R(1)}^{(14)}=\left(U^{T} \Delta_{R}^{t \dagger} \Delta_{R}^{t} U\right)_{55}=\frac{4}{5}-\frac{3}{4} \sin ^{2}\left(h / f_{\pi}\right) \\
& v_{R(2)}^{(14)}=\left(U^{T} \Delta_{R}^{t \dagger} U\right)_{55}\left(U^{T} \Delta_{R}^{t} U\right)_{55}=\frac{4}{5}-\frac{1}{4}\left(3+5 \cos ^{2}\left(h / f_{\pi}\right)\right) \sin ^{2}\left(h / f_{\pi}\right) .
\end{aligned}
$$

The symmetric representation has the crucial feature that the combinations of the type $\left(U^{T} \Delta U\right)_{55}$, which vanished before, now deliver a finite result. On the one hand, these allow to mix $U_{5 i}, i=1, \ldots, 4 \sim \sin \left(h / f_{\pi}\right)$ with $U_{55} \sim \cos \left(h / f_{\pi}\right)$ via a single insertion of $\left(\Delta_{L}^{t}\right)_{\alpha}$, delivering directly the new $\left(\sin \left(h / f_{\pi}\right) \cos \left(h / f_{\pi}\right)\right)^{2}$ invariant, on the other hand they provide single trigonometric functions to the fourth power via only two insertions of $\Delta_{R}^{t}$, resulting in contributions to the same invariant.

As a consequence, we have

$$
\begin{aligned}
V_{2}^{(14)}(h) \cong & \frac{N_{c} m_{\Psi}^{4}}{16 \pi^{2} g_{\psi}^{2}}\left[-\frac{3}{4}\left(c_{L}^{t(1)} y_{L}^{t 2}+\left(c_{R}^{t(1)}+c_{R}^{t(2)}\right) y_{R}^{t 2}\right)\right] \sin ^{2}\left(h / f_{\pi}\right) \\
& -\frac{N_{c} m_{\Psi}^{4}}{16 \pi^{2} g_{\psi}^{2}}\left[-c_{L}^{t(2)} y_{L}^{t 2}+\frac{5}{4} c_{R}^{t(2)} y_{R}^{t}\right] \sin ^{2}\left(h / f_{\pi}\right) \cos ^{2}\left(h / f_{\pi}\right)
\end{aligned}
$$

and thus can accommodate a realistic EWSB just with $V_{2}^{(14)}(h)$ - in principle without an additional tuning. The coefficients $\alpha$ and $\beta$ (see (2.19)) are both generated at $\mathcal{O}\left(\Delta^{2}\right)$ and so are generically of the same order. In consequence (2.21) can be solved in a nontrivial way without relying on formally subleading contributions and in particular for various 
hierarchies between $y_{L}^{t}$ and $y_{R}^{t}$, keeping still $c_{L, R}^{t(1),(2)} \sim \mathcal{O}(1) .{ }^{14}$ Since we do not anymore need to artificially cancel one order in $y_{L, R}^{t 2} / g_{\Psi}^{2}$ in $\alpha$, the formal tuning in EWSB is reduced to the minimal amount of

$$
\Delta_{(14)}^{-1} \sim \sin ^{2}\left(v / f_{\pi}\right) .
$$

In contrast to the $\mathrm{MCHM}_{5,10}, \mathcal{O}(1)$ changes in the parameters on the left hand side of $(2.21)$ induce only moderately large changes in $v / f_{\pi}$, while the space of viable solutions is not directly left.

For the Higgs mass we obtain (see (2.23))

$$
m_{H}^{2} \stackrel{\mathrm{MCHM}_{14}}{=} m_{\Psi}^{2} \frac{N_{c}}{2 \pi^{2}}\left(-c_{L}^{t(2)} y_{L}^{t 2}+\frac{5}{4} c_{R}^{t(2)} y_{R}^{t 2}\right) \cos ^{2}\left(v / f_{\pi}\right) \sin ^{2}\left(v / f_{\pi}\right),
$$

and thus in general

$$
m_{H}^{(14)} \sim \sqrt{\frac{3}{2 \pi^{2}}} \sqrt{\left|\frac{5}{4} y_{R}^{t 2}-y_{L}^{t 2}\right|} \frac{m_{\Psi}}{f_{\pi}} v \sim \frac{m_{\Psi}}{f_{\pi}} v,
$$

where the last similarity holds if at least one of the $y_{L, R}^{t} \sim \mathcal{O}(1)$, as expected due to the large $m_{t}$, and no unnatural cancellation is happening. We observe that the Higgs boson is in general significantly heavier than the electroweak scale in this model. In particular, it is heavier by a factor of $\sim m_{\Psi} / f_{\pi}=g_{\Psi}$ with respect to the $\mathrm{MCHM}_{5}$ and the $\mathrm{MCHM}_{10}$, see (2.24). Remember that $m_{\Psi}$ is the generic scale of the (heavy) fermionic resonances and in general not the one of the potentially light partners. Finally, in the $\mathrm{MCHM}_{14}$ light partners can not help to reduce $m_{H}$ up to the experimental value, since even such partners can not allow $y_{L, R}^{t}$ to become very much smaller than one - then the top mass could not be reproduced any longer. The general scale (2.44) is just too large [10].

In the end, while in the $\mathrm{MCHM}_{5,10}$ (in the $\mathrm{MCHM}_{5,10}$ with light top partners) the Higgs is naturally expected to reside at (slightly below) the electroweak scale, $m_{H} \sim v\left(m_{H}<v\right)$, in the $\mathrm{MCHM}_{14}$ as discussed above a significant ad hoc tuning of in general unrelated parameters in $(2.43)$ is needed in order to push the Higgs mass to $m_{H} \lesssim v$. Note in particular that while EWSB is a necessary condition for the universe being able to host human beings, a heavier Higgs scenario could in principle be as viable as the light-Higgs one, which justifies to consider the tuning in the $\mathrm{MCHM}_{14}$ really as ad hoc, in comparison with the one in the $\mathrm{MCHM}_{5,10}$. Although, due to the particular numerical value of the Higgs mass, in the end the total tuning in both classes of models turns out to be not too different (see section 3), for the $\mathrm{MCHM}_{14}$ a light Higgs boson as found seems more unnatural. ${ }^{15}$

On the other hand, as we will see in particular in the numerical analysis, in the $\mathrm{MCHM}_{14}$ one has indeed enough freedom to tune the parameters such as to accommodate a light Higgs mass without necessarily light top partners. This tuning also does not need to spoil the power counting in $y^{2} / g_{\Psi}^{2}$. In that context, remember that the other option

\footnotetext{
${ }^{14}$ The latter fact will be interesting for constructing minimal models featuring a $\mathbf{1 4}$, since essentially only one chirality is needed for a viable EWSB, see below.

${ }^{15}$ Note also that the tuning in the $\mathrm{MCHM}_{14}$ has to be present both in $\beta$ and $\alpha$ and that light partners can only reduce it marginally by allowing for somewhat smaller $y_{L, R}^{t}$.
} 
of realizing a light Higgs with the help of light top partners in the $\mathrm{MCHM}_{5,10}$ is already under pressure from LHC searches and could be excluded soon. As a consequence, taking only into account the quark sector, involving a $\mathbf{1 4}$ with a relatively "unnatural" light Higgs with respect to its actual scale, might be the last option for the composite Higgs to hide. We will now survey the most economical realizations of that idea.

MCHM $_{14-\mathbf{X}}, \mathbf{M C H M}_{\mathbf{X - 1 4}}$. Already if only one SM-quark chirality mixes with a $\mathbf{1 4}$ of $\mathrm{SO}(5)$ and the other with any of the representations, the main qualitative features discussed above remain valid. The same remains true if the SM quarks mix with more than one representation at the same time, adding more $\Psi$ fields to (2.4), as long as one $\mathbf{1 4}$ is present (with a sizable composite component). Only the numerical $\mathcal{O}(1)$ coefficients in front of the linear mixing parameters $y_{L, R}^{t}$ in (2.41) will change (with potentially the contribution of one chirality vanishing or additional mixings entering), while necessarily still both trigonometric structures will emerge at leading order in the spurions, thus avoiding the double tuning. In particular, the expression for the Higgs mass (see $(2.43),(2.44)$ ) will remain as a dominant term, due to the unsuppressed contribution of the 14 to $\beta$ - so at least the requirement of some ad-hoc tuning remains. Mixings with other representations can have an impact on the final numerical value of the Higgs mass (after ad hoc tuning), however do not change the general picture.

In particular, the most minimal quark model avoiding the necessary presence of light top partners becomes evident, the $\mathrm{MCHM}_{14-1}$, i.e., the $t_{L}$ mixing with a $\mathbf{1 4}$ and the $t_{R}$ with a singlet $[9,10,56]$, see also section 3 . Note that only if a $\mathbf{1 4}_{L}$ is involved, guaranteeing EWSB by itself, it is possible to mix the $t_{R}$ with a singlet of $\mathrm{SO}(5)$, thus not contributing to the breaking of $\mathrm{SO}(5)$. Now, eqs. (2.41)-(2.44) remain valid in the limit where the contributions $\sim y_{R}^{t}$ are set to zero. Let us finish this subsection with adding up the degrees of freedom (dof) in the complete composite-fermion particle spectrum of this minimal model avoiding the presence of light fermion partners, in units of dirac fermions (neglecting color so far). Taking into account the bottom sector in the most minimal way $\left(\mathbf{5}_{b_{L}}+\mathbf{1}_{b_{R}}\right)$ as well as the most minimalistic lepton embedding $\left(\mathbf{5}_{\tau_{L}}+\mathbf{1}_{\tau_{R}}+\mathbf{5}_{\nu_{L}}+\mathbf{1}_{\nu_{R}}\right)$, we arrive at a total number of $(14+1+5+1)_{q}+(5+1+5+1)_{l}=21_{q}+12_{l}=33$ particles. $^{16}$

In the following subsection we will introduce a new class of models to avoid the presence of ultra-light fermionic partners, allowing on the one hand side to avoid the ad hoc tuning, while on the other hand they can even even increase the naturalness of the assumptions, the minimality, and in particular the predictivity with respect to this setup. We are lead to these models by considering for the first time the impact of a realistic lepton sector on the Higgs potential, making use of the formulae presented in this subsection.

\subsection{The impact of the leptonic sector}

Since leptons are present in nature, they need to be included into the composite Higgs framework. Naively, due to their small masses, one might expect the impact of leptons on the Higgs potential to be small. However, as explained before and as is well known, from

\footnotetext{
${ }^{16}$ Note that in general the most minimal embedding of the quark sector, neglecting a potential impact of leptons on $V(h)$, is $5_{t_{L}}+5_{t_{R}}+5_{b_{L}}+1_{b_{R}}$ or $10_{q_{L}}+5_{t_{R}}+1_{b_{R}}$ with 16 dof, compared to the 21 above.
} 
the flavor structure in the lepton sector there is compelling motivation to assume the $\tau_{R}$ to be rather composite and thus coupled more significantly to the Higgs than suggested by the small mass of the $\tau$.

Beyond that, as we will show more explicitly in section 3.1.4, already the tiny neutrino masses provide a motivation for an enhanced compositeness in the whole right-handed lepton sector. The seesaw mechanism can be elegantly embedded in the composite framework, via a large Majorana mass in the elementary sector. Since in such models the natural value for this mass is however the Planck scale [12, 57-60], the right-handed neutrinos should feature a non-negligible composite component that reduces their coupling to the Majorana-mass term by several orders of magnitude and thus should have a rather large $y_{R}^{\nu}$. As we will show, in very minimal setups for the lepton sector this is mirrored to a sizable $y_{R}^{\ell}$ for the charged leptons.

In particular this is the case for the model that we will advocate and examine in more detail in section 3.1.4, where the complete SM lepton sector will only mix with a single $\mathbf{5}_{L}+\mathbf{1 4}_{R}$ (for each generation). While the left-handed doublet will couple to a minimal $\mathbf{5}$, right-handed neutrinos as well as charged leptons will mix with the same 14, realizing a type-III seesaw mechanism in the most minimal way that allows for a protection of the $Z \tau_{R} \tau_{R}$ couplings. Moreover, this model features the least number of parameters possible for a realistic embedding of the lepton sector in general.

In the following we will give a comprehensive overview of the impact of possible realizations of the full fermion sector on the Higgs potential, the tuning, and the emergence of light partners, always assuming the $\tau_{R}$ to feature a non-negligible compositeness. We will again focus on the third generation for simplicity, while we will comment on the other generations relevant for the type-III seesaw model later.

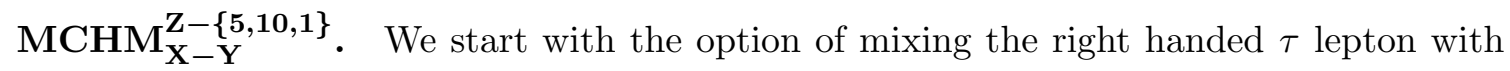
either a fundamental, an adjoint, or a singlet representation of $\mathrm{SO}(5)$, as denoted by $\{5,10,1\}$, where here and in the following the lepton representations are always given by superscripts. The left handed leptons as well as quarks are on the other hand allowed to mix with any of the representations considered. ${ }^{17}$ In that case the $\tau_{R}$ contributions to $\alpha$ and $\beta$ in (2.19), denoted as $\alpha_{\tau}, \beta_{\tau}$, arise at most at the same order in $y_{L, R}^{\tau, t} / g_{\Psi}$ as the top contribution. Now we need to note that the lepton contribution at a certain order is in general considerably smaller than the quark contribution. It adds a similar term to $V_{2}(h)$ as given in (2.17) or in (2.32), with however

$$
N_{c} \rightarrow 1, \quad y_{L}^{t, q} \rightarrow 0, \quad t \rightarrow \tau
$$

(as well as to $V_{4}(h)$ with the same replacements in (2.18) and (2.34)). First of all, this is not $N_{c}$ enhanced. Beyond that, the $c_{R(R R)}^{\tau(\ldots)}$, comprising of mass-related quantities $a_{L}^{\tau}, b_{R}^{\tau}$, etc.

\footnotetext{
${ }^{17}$ Remember that both the right-handed bottom quark and neutrino are not important for the Higgs potential and will thus not be considered explicitly. We will nevertheless keep them in mind for the complete setup of the model, where we will include right handed neutrinos with the neutrino masses originating either from a (type I or III) seesaw mechanism or from a pure Dirac mass. The contribution of the left handed leptons will also always be negligible, due to their small compositeness.
} 
(see $(2.12)$, with $(t, T) \rightarrow(\tau, \mathcal{T}))$, are in general somewhat smaller than $c_{R(R R)}^{t(\ldots)}$ in viable lepton models, see section 3 . As a consequence the impact of the lepton sector on the Higgs mass via $\beta_{\tau}$ is directly negligible to good approximation. Regarding the condition from EWSB (2.21), the lepton contribution $\alpha_{\tau}$ is also significantly suppressed with respect to the quark contribution in general. Only after $\alpha_{t}$ has been tuned to be of the order of $\beta_{t}$ to guarantee EWSB, see section 2.2, $\alpha_{\tau}$ might become relevant for the numerical minimization condition. It however does not change any of the qualitative conclusions in section 2.2. The same is true in general for the subleading contribution $\alpha_{W}$ of the gauge bosons.

$\operatorname{MCHM}_{\{\mathbf{5 , 1 0}-\{\mathbf{5 , 1 0 , 1 \}}}^{\mathbf{Z}-\mathbf{1 4}}$. We now move forward to the case of the $\tau_{R}$ mixing with a symmetric representation of $\mathrm{SO}(5)$, where we will find interesting new features. The left handed SM-like quarks will first be restricted to mix with either a fundamental or an adjoint representation, while the right handed quarks could alternatively also mix with a singlet. The relevant contributions to the Higgs potential now look like

$$
\begin{aligned}
V(h) \cong & {\left[\frac{m_{\Psi}^{4}}{16 \pi^{2} g_{\psi}^{2}}\left(N_{c}\left(\tilde{c}_{L}^{t} y_{L}^{t 2}-\tilde{c}_{R}^{t} y_{R}^{t 2}\right)+\tilde{c}_{R}^{\tau} y_{R}^{\tau 2}\right)+\alpha_{t}^{(4)}\left(y_{L, R}^{t 4} / g_{\Psi}^{4}\right)\right] \sin ^{2}\left(h / f_{\pi}\right) } \\
& -\left[\frac{m_{\Psi}^{4}}{16 \pi^{2} g_{\psi}^{2}} 5 / 4 c_{R}^{\tau(2)} y_{R}^{\tau 2}+\beta_{t}\left(y_{L, R}^{t 4} / g_{\Psi}^{4}\right)\right] \sin ^{2}\left(h / f_{\pi}\right) \cos ^{2}\left(h / f_{\pi}\right)
\end{aligned}
$$

Here $\tilde{c}_{L}=\left\{c_{L}^{t} / 2,-3 / 8 c_{L}^{q}\right\}$ in the case of $t_{L}$ mixing with a $\{\mathbf{5}, \mathbf{1 0}\}$ of $\mathrm{SO}(5)$, whereas $\tilde{c}_{R}=\left\{c_{R}^{t},-1 / 4 c_{R}^{t}, 0\right\}$ for $t_{R}$ mixing with a $\{\mathbf{5}, \mathbf{1 0}, \mathbf{1}\}$, respectively, see (2.17) and (2.32), while simply from $(2.41)$ we identify $\tilde{c}_{R}^{\tau}=-3 / 4\left(c_{R}^{\tau(1)}+c_{R}^{\tau(2)}\right)$. The subleading quark terms, contributing to $\beta_{t}\left(y_{L, R}^{t 4} / g_{\Psi}^{4}\right)$ can be obtained easily from (2.18) $((2.34))$ for the case of $t_{L}$ and $t_{R}$ both mixing with a $5(\mathbf{1 0})$ of $\mathrm{SO}(5)$. The same is true for the $\mathcal{O}\left(y_{L, R}^{t 4} / g_{\Psi}^{4}\right)$ contributions to $\alpha_{t}$, denoted as $\alpha_{t}^{(4)}$. In any case, a new trigonometric function beyond the $\sin ^{2}\left(h / f_{\pi}\right)$ emerges from the quark sector only at $\mathcal{O}\left(y_{L, R}^{t 4} / g_{\Psi}^{4}\right)$.

We inspect that the lepton sector can now deliver an essential contribution. First of all, it provides the formally leading term in the second row of (2.46), contributing to $\beta$ and thus to the Higgs mass at $y^{2} / g_{\Psi}^{2}$. Although, as discussed before, the lepton sector features a notable general suppression with respect to the top quark contributions to the potential, this can be lifted by the smaller power suppression in $y^{2} / g_{\Psi}^{2}$ compared to $\beta_{t}$. The $\tau_{R}$ contribution can thus help to allow for a light Higgs boson with $m_{H} \sim 125 \mathrm{GeV}$, without the need for light top partners. The crucial point is that now we add two new mixing parameters $y_{L}^{\tau}$ and $y_{R}^{\tau}$ (as well as additional $(\mathcal{O}(1))$ mass parameters) but only one new constraint, i.e., the $\tau$ mass. Although only the latter mixing is relevant for the potential, this however now is basically a new free parameter.

Without leptons, all mixing parameters were determined to good approximation by the top mass (2.14) and the EWSB condition (2.21), where the latter fixed $y_{L}^{t} \sim y_{R}^{t} \equiv y_{t}$, as explained before. Like this, $m_{H}$ was essentially fixed and generically too large, see e.g. (2.25). The only additional freedom for a potential reduction in the Higgs mass was to lower the top-partner masses $m_{T}$ or $m_{\tilde{T}}$, to increase the mixing with the resonances which allows to lower $y_{t}$ for fixed $m_{t}$ and thus to lower $m_{H}$. Now, the leptonic contribution offers 
an opportunity to break this pattern by providing the additional parameter $y_{R}^{\tau}$ entering $m_{H}$. Adding $\beta_{\tau}$ to $(2.23)$ we arrive at (setting $c_{L L, L R, R R}^{t} \rightarrow 1$ )

$$
\begin{aligned}
m_{H} & \sim \frac{1}{\sqrt{2} \pi} \sqrt{3 y_{t}^{4}+5 / 4 c_{R}^{\tau(2)} g_{\Psi}^{2} y_{R}^{\tau 2}} v \\
& \sim \frac{1}{\pi} \sqrt{3 \frac{\min \left(m_{T}, m_{\tilde{T}}\right)^{2}}{f_{\pi}^{2}} m_{t}^{2}+5 / 8 c_{R}^{\tau(2)} g_{\Psi}^{2} y_{R}^{\tau 2} v^{2}},
\end{aligned}
$$

and similarly for the other non-trivial quark embeddings mentioned before, while we will comment on the option of embedding the $t_{R}$ in a singlet further below. Since the lepton sector delivers a non-negligible contribution to $m_{H}$, one can now use the additional freedom to arrive at the correct Higgs mass, without the need to tune the top partners light, only by moderately canceling the top contribution in (2.47) above via the contribution of the $\tau_{R}$. In section 3 we will see explicitly that the effect of the latter is significant and can indeed interfere destructively with the top contribution, to allow for larger $\min \left(m_{T}, m_{\tilde{T}}\right)$.

Moreover, in contrast to quarks in the $\mathbf{1 4}$, no large ad-hoc tuning is needed, since in general $\beta_{\tau}$ is significantly smaller than $\beta_{t}$ when the fermions mix with a $\mathbf{1 4}$. In the case of quarks, the ad hoc tuning was unavoidably generated due to the large top mass. With the quarks now mixing with a fundamental or adjoint representation of $\mathrm{SO}(5)$, their contribution to $\beta$ is suppressed one order further in $y_{t}^{2} / g_{\Psi}^{2}$ such that both the quark and the lepton contribution are already roughly as small as the electroweak scale (cf. (2.44)). There is no need for light resonances whatsoever. Finally, the impact of the $\tau_{R}$ on the EWSB condition (2.21) is in general still similar to the case discussed before. It is subleading in the first place, while after the tuning in the quark sector, which still more or less leads to $y_{L}^{t} \sim y_{R}^{t}$ (see first line of (2.46)), it can have a modest impact on the numerics. We will see the whole mechanism at work explicitly in section 3 .

$\mathbf{M C H M}_{\mathbf{1 4 - Y}}^{\mathbf{Z}-\mathbf{W}}, \mathbf{M C H M}_{\mathbf{X}-\mathbf{1 4}}^{\mathbf{Z}-\mathbf{W}}$. For completeness, we now discuss the case where the $t_{L}$ or the $t_{R}$ mixes with a $\mathbf{1 4}$ of $\mathrm{SO}(5)$, while the other fermions can mix with any one of the representations considered. Now the leptonic effect is again subleading in general, since the top contributions are always non-vanishing at the maximal possible order $\left(y^{2} / g_{\Psi}^{2}\right)$, see section 2.2. Nevertheless the $\tau_{R}$ can have e.g. a numerical impact on the Higgs mass, if it mixes itself with a $\mathbf{1 4}$, after the tuning of the large quark contribution in order to reproduce the small $m_{H}$. After this reduction in the quark contribution, the leptons can also have again a numerical influence on the EWSB condition for various representations. However, they again deliver no qualitative change of the overall mechanism and findings with respect to the tuning and the absence of light partners if quarks are in the $\mathbf{1 4}$.

In general, we have shown that if a composite $\tau_{R}$ mixes with a symmetric representation of $\mathrm{SO}(5)$, interesting consequences can arise. In particular, light top partners are no longer needed, while still a large tuning in the Higgs mass can be avoided. We will see now that such models of the lepton sector can also have very interesting features regarding the minimality of the setup. As explained, models of $\tau$ compositeness are well motivated from lepton-flavor physics, in particular from the absence of sizable FCNCs. However, the fact that leptons should mix with a $\mathbf{1 4}$ of $\mathrm{SO}(5)$ is at this stage still rather ad hoc. In the end, 
although the setup offers an orthogonal approach and clearly has some virtues with respect to the $\mathbf{1 4}$ in the quark sector, so far it is has not been proven to be more motivated from a conceptual reasoning.

On the other hand, due to the mere fact that the neutrino masses are so tiny, a very attractive motivation for having leptons in a $\mathbf{1 4}$ of $\mathrm{SO}(5)$ can be given, even without the need to rely on flavor protection to justify right-handed lepton compositeness. Indeed, the $\mathrm{SU}(2)_{L}$ triplet present in the $\mathbf{1 4}$ is very welcome regarding neutrino masses. If it acquires a Majorana mass term, it provides heavy degrees of freedom that can induce the strongly suppressed dimension-5 Weinberg operator, responsible for generating tiny neutrino masses via the well known (type-III) seesaw mechanism. As mentioned above, the $\mathbf{5}_{L}+\mathbf{1 4}_{R}$ setup provides the most minimal composite Higgs model with a type-III seesaw for neutrino masses (with a protection of $Z \tau_{R} \tau_{R}$ ) and will thus be denoted the mMCHM ${ }^{\mathrm{III}}$. Beyond that minimality with respect to the particle content, since both the heavy lepton triplet containing the right-handed Majorana neutrino as well as the $\tau_{R}$ can mix with the same composite multiplet (containing both a $\mathrm{SU}(2)_{L}$ triplet and singlet), the model features the least number of parameters possible, even in general. ${ }^{18}$ Finally, note that in that context a similar type-I seesaw model would need at least an additional $\mathbf{1}_{\nu_{R}}$ and in consequence another $\mathbf{5}_{L}$, and would thus be much less minimal. Interestingly, the lack of light partners together with minimality points to a type-III seesaw model.

Moreover, an equivalently simple model with a $\mathbf{1 4}$ in the quark sector is not possible. It is a peculiar feature of the lepton sector that the right-handed neutrinos can be part of a $\mathrm{SU}(2)_{L}$ triplet, while for quarks the right handed fields need both to mix with $\mathrm{SU}(2)_{L}$ singlets which requires a second composite multiplet (beyond the 14) mixing with the right handed fermions, complicating the model. ${ }^{19}$

In that context, note that for the $\mathrm{mMHCM}^{\mathrm{III}}$, considering the embeddings of the quarks, the very minimal and in principle obvious possibility of mixing the $\mathrm{SU}(2)_{L}$ singlet $t_{R}$ with a singlet of $\mathrm{SO}(5) \supset \mathrm{SU}(2)_{L}$ arises naturally, while still the $t_{L}$ can mix with a $\mathbf{5}$ - the $\mathrm{mMCHM}_{5-1}^{\mathrm{III}>}$. This now becomes viable due to a potentially unsuppressed leptonic contribution in the $\mathrm{mMCHM}^{\mathrm{III}}$, which here can become larger than in the models of $\tau_{R}$ compositeness considered so far. First, the $N_{c}=3$ suppression can be lifted via an $N_{g}=$ 3 enhancement, since now all right handed charged-lepton generations are expected to contribute (see section 3.1.4). Moreover, remember that the model does not need anymore to be based on a specific setup of flavor protection, coming usually with smaller $c_{R(R R)}^{\tau(\ldots)}$ from a 'Yukawa suppression' [12], to motivate a large right-handed lepton compositeness.

\footnotetext{
${ }^{18}$ Note that if the right-handed $\tau$ and the $N_{R}$ mix with the same multiplet of the strong sector, this suggests that they feature the same linear-mixing parameter (determined by the anomalous dimension of the composite operator) $y_{R}^{\tau}=y_{R}^{N_{\tau}}$, where the latter should be sizable, as explained before. The new fields that the $\mathbf{1 4}$ offers are thus used in an economical way. A further reduction in parameters (and fields) with respect to the $\mathrm{MCHM}_{14}$ is reached since, with both right handed lepton multiplets being able to mix with the same composite representation with a single $X$ charge, also the left handed fields can mix with a single representation (here a 5). As a consequence, the setup involves the minimal amount of composite $\mathrm{SO}(5)$ multiplets that is viable - less than any other model known before.

${ }^{19}$ While in the 10 it would in principle be possible to embed both $t_{R}$ and $b_{R}$, this is in any case disfavored, as it would introduce large corrections to $Z b_{R} \bar{b}_{R}$ couplings due to the large top mass.
} 
The lifting of that suppression is denoted by the superscript $>$. In this setup all terms $\sim y_{R}^{t}$ in (2.46) vanish and it becomes possible that the now sizable $\tau_{R}$ contribution to $\alpha$ moderately cancels the $t_{L}$ one, such as to fulfill (2.21).

In particular $y_{L}^{t}$ can also be somewhat smaller if the $t_{R}$ mixes with a singlet, since the top mass can always be matched via the now free $y_{R}^{t}$, which is not required to fulfill $y_{R}^{t} \sim y_{L}^{t}$ anymore from EWSB. This will just lead to a strongly composite $t_{R}$. In consequence of this freedom, the contribution from the quark sector to the Higgs mass is also no longer fixed by $m_{t}$ and can become somewhat smaller, without light partners. Now generically $m_{H}$ becomes dominated by $\beta_{\tau}$ and the leptons do not need to interfere destructively with the quark contribution to $\beta$ anymore. Due to the generically large $\beta_{\tau}$ the model will feature an ad-hoc tuning, similar to the model with a $\mathbf{1 4}_{t_{L}}+\mathbf{1}_{t_{R}}$, however the parametric "double-tuning" in EWSB can clearly be avoided, although the quark realization is minimal. Note that in such a scenario of a unsuppressed contribution of a leptonic $\mathbf{1 4}_{R}$ to $\alpha$ and $\beta$, the lepton effects can help to avoid the "double-tuning" (and the emergence of light partners) also in other realizations of the quark sector than the $\mathbf{5}_{L}+\mathbf{1}_{R}$, breaking also the $y_{L}^{t} \sim y_{R}^{t}$ degeneracy in general. In all models, besides those featuring a $\mathbf{1 4}$ in the quark sector, the leptonic contributions, discussed in (2.47), will then generically dominate $m_{H}$ before necessary cancellations take place. If quarks are however in a $\mathbf{1 4}$, both sectors become comparable and the pure quark models are only modified in a sense that now all contributions become larger in general due to the additional lepton terms.

On the other hand, note that also in the $\mathrm{mMCHM}^{\mathrm{III}}$ it is still possible to motivate a Yukawa suppression for leptons, which will here be balanced by a slightly enhanced lefthanded compositeness to keep the lepton masses fixed. This is in particular feasible since, in contrast to the large top mass, one has $m_{\tau} \ll v$. This leads to the other limit of the model, which results in rather similar predictions as for the models with a simple $\mathbf{1 4}_{\tau_{R}}$, described before. The most minimal viable version of this setup features a $\mathbf{5}_{t_{L}}+\mathbf{5}_{t_{R}}$ in the top-quark sector, while the bottom sector (and light quarks) can be in a $\mathbf{5}_{b_{L}}+\mathbf{1}_{b_{R}}$, and will be denoted the $\mathrm{mMCHM}_{5}^{\mathrm{III}}$, while the corresponding optional version with large lepton Yukawas will again be called $\mathrm{mMCHM}_{5}^{\mathrm{III}>}$.

Nevertheless, the most minimal complete model which avoids the presence of light top-partners belongs to the class $\mathrm{MCHM}_{5-1}^{5-14}$, where the full embedding reads $\mathbf{5}_{t_{L}}+\mathbf{1}_{t_{R}}+\mathbf{5}_{b_{L}}+\mathbf{1}_{b_{R}}+\mathbf{5}_{\tau_{L}}+\mathbf{1 4}_{\ell_{R}}$, equipped with a Majorana mass for the $\mathrm{SU}(2)_{L}$ triplet and unsuppressed lepton Yukawas. It is just this model, featuring 31 dof, that we will think of as the $\mathrm{mMCHM}_{5-1}^{\mathrm{III}>}$ in the following. As discussed, the quarks can now mix with composites with only 12 dof. $^{20}$ The model has thus less dof in the quark sector than any other composite model known, minimizing the colored particle content, with further interesting consequences for phenomenology.

The most minimal model that avoids the presence of ultra-light partners by a modification of the quark sector is on the other hand defined as $\mathbf{1 4}_{t_{L}}+\mathbf{1}_{t_{R}}+\mathbf{5}_{b_{L}}+\mathbf{1}_{b_{R}}+\mathbf{5}_{\tau_{L}}+$ $\mathbf{1}_{\tau_{R}}+\mathbf{5}_{\nu_{L}}+\mathbf{1}_{\nu_{R}}$. Beyond the fact that the $\mathbf{1 4}$ in such a model is conceptually less motivated

\footnotetext{
${ }^{20}$ This corresponds to the minimal possible amount of fermions one might think of in general $\mathrm{SO}(5) / \mathrm{SO}(4)$ composite Higgs models, whereas the minimum for a sector that needs to trigger EWSB was $5_{t_{L}}+1_{t_{R}}+$ $5_{b_{L}}+1_{b_{R}}=16=5_{q_{L}}+10_{t_{R}}+1_{b_{R}}$.
} 
from the pattern of the quark masses and seems ad hoc, it has 33 dof, and thus more than the $\mathrm{mMCHM}_{5-1}^{\mathrm{III}>}$ where the $\mathbf{1 4}$ is used to unify different SM multiplets. If nature should call for a $\mathbf{1 4}$ of $\mathrm{SO}(5)$ via the non-discovery of light top partners, the lepton sector with a type-III seesaw seems to offer the most economical place to host this multiplet.

In summary, models with leptons in a symmetric representation of $\mathrm{SO}(5)$, such as the $\mathrm{mMCHM}_{5}^{\mathrm{III}}$ offer the new possibility to create a naturally light Higgs without light partners in a well motivated setup following the principle of minimality in the lepton sector, while the total dof are in the ballpark of minimal models (already the standard $\mathrm{MCHM}_{5}$ with all fermions in a $\mathbf{5}$ has more). Moreover, they also invite to set up models with a maximal total amount of minimality, by allowing the most minimal and natural quark embedding, with right handed singlets of $\mathrm{SO}(5) \supset \mathrm{SU}(2)_{L}$ only, the $\mathrm{mMCHM}_{5-1}^{\mathrm{III}}$. Counting color as a degree of freedom, such a model features $3 \cdot 12_{q}+19_{l}=55$ fermionic dof for the third generation, compared to the most minimal viable model known before, with $3 \cdot 16_{q}+12_{l}=60$ fermionic dof. ${ }^{21}$ It could thus be considered as the most minimal composite Higgs model in general and it does not predict light partners. In consequence, minimality as an argument to expect light partners at the LHC might be questionable. In the following we will quantify the general findings of this section.

\section{Numerical analysis in the GHU approach - the impact of leptons}

In this section we are going to probe the general findings of last section numerically by studying explicit holographic realizations of the composite Higgs setup, i.e., models of gauge-Higgs unification [19-24]. We will first present the 5D framework for the models under consideration and then discuss the calculation of the Higgs potential. Finally we will present our numerical results and confront them with the general predictions of section 2 .

\subsection{Setup of the (5D) GHU models}

The 5D holographic realization of the MCHMs introduced in the previous section consists of a slice of $\mathrm{AdS}_{5}$ with metric

$$
\mathrm{d} s^{2}=a^{2}(z)\left(\eta_{\mu \nu} \mathrm{d} x^{\mu} x^{\nu}-\mathrm{d} z^{2}\right) \equiv\left(\frac{R}{z}\right)^{2}\left(\eta_{\mu \nu} \mathrm{d} x^{\mu} x^{\nu}-\mathrm{d} z^{2}\right),
$$

where $z \in\left[R, R^{\prime}\right]$ is the coordinate of the additional spatial dimension and $R$ and $R^{\prime}$ are the positions of the UV and IR branes, respectively. We consider a bulk gauge symmetry $\mathrm{SO}(5) \times \mathrm{U}(1)_{X}$ broken by boundary conditions to the electroweak group $\mathrm{SU}(2)_{L} \times \mathrm{U}(1)_{Y}$ on the UV brane and to $\mathrm{SO}(4) \times \mathrm{U}(1)_{X}$ on the IR one. More explicitly, this setup correspond to gauge bosons with the following boundary conditions

$$
L_{\mu}^{a}(+,+), \quad R_{\mu}^{b}(-,+), \quad B_{\mu}(+,+), \quad Z_{\mu}^{\prime}(-,+), \quad C_{\mu}^{\hat{a}}(-,-),
$$

where $a=1,2,3, b=1,2, \hat{a}=1,2,3,4$ and $-/+$ denote Dirichlet/Neumann boundary conditions at the corresponding brane. ${ }^{22}$ In the above expression, $L_{\mu}^{1,2,3}$ and $R_{\mu}^{1,2,3}$ are

\footnotetext{
${ }^{21}$ The same counting goes trough to the case of three generations in the fully anarchic approach to flavor.

${ }^{22}$ The respective $(4 \mathrm{D})$ scalar components $(\mu \rightarrow 5)$ have opposite boundary conditions, allowing for zero modes only in $C_{5}^{\hat{a}}$.
} 
the $4 \mathrm{D}$ vector components of the $5 \mathrm{D}$ gauge bosons associated to $\mathrm{SU}(2)_{L}$ and $\mathrm{SU}(2)_{R}$, respectively, both subgroups of $\mathrm{SO}(5)$. We have defined the linear combinations

$$
\begin{aligned}
B_{\mu} & =s_{\phi} R_{\mu}^{3}+c_{\phi} X_{\mu}, & Z_{\mu}^{\prime} & =c_{\phi} R_{\mu}^{3}-s_{\phi} X_{\mu}, \\
c_{\phi} & \equiv \frac{g_{5}}{\sqrt{g_{5}^{2}+g_{X}^{2}}}, & s_{\phi} & \equiv \frac{g_{X}}{\sqrt{g_{5}^{2}+g_{X}^{2}}},
\end{aligned}
$$

with $g_{5}$ and $g_{X}$ the dimensionfull 5D gauge couplings of $\mathrm{SO}(5)$ and $\mathrm{U}(1)_{X}$, respectively, and $X_{\mu}$ the gauge boson associated with the additional $\mathrm{U}(1)_{X}$. Finally, $C_{\mu}^{\hat{a}}$ are the gauge bosons corresponding to the broken coset space $\mathrm{SO}(5) / \mathrm{SO}(4)$, whose scalar counterparts zero-modes $C_{5,(0)}^{\hat{a}}(x, z) \equiv f_{h}^{\hat{a}}(z) h^{\hat{a}}(x)$ we will identify with the $\mathrm{SU}(2)_{L}$ Higgs doublet.

We typically fix $1 / R \sim 10^{16} \mathrm{TeV}$ and, for each value of the warped down $1 / R^{\prime} \sim$ $\mathcal{O}(1) \mathrm{TeV}$, addressing the hierarchy problem, we obtain $g_{5}, s_{\phi}$ and $\left\langle h^{\hat{a}}\right\rangle=v \delta_{\hat{a} 4}$ in terms of $\alpha_{\mathrm{QED}}, M_{W}$ and $M_{Z}$. That means that, modulo the value of $R \sim M_{\mathrm{Pl}}^{-1}$, fixed by naturalness, in the $5 \mathrm{D}$ gauge sector the only free parameter is $R^{\prime}$, or equivalently,

$$
f_{\pi} \equiv \frac{2 R^{1 / 2}}{g_{5} R^{\prime}}
$$

We will also consider the more general scenario where the dimensionless $5 \mathrm{D}$ gauge coupling $g_{*} \equiv g_{5} R^{-1 / 2}$ (as well as $s_{\phi}$ ) can change for a fixed value of $f_{\pi}$. This can be done by allowing for $\mathrm{SU}(2)_{L}$ and $\mathrm{U}(1)_{Y} \mathrm{UV}$ localized brane kinetic terms,

$$
\mathcal{S}_{\mathrm{UV}} \supset \int \mathrm{d}^{4} x\left[-\frac{1}{4} \kappa^{2} R \log \left(\frac{R^{\prime}}{R}\right) L^{a \mu \nu} L_{\mu \nu}^{a}-\frac{1}{4} \kappa^{\prime 2} R \log \left(\frac{R^{\prime}}{R}\right) B^{\mu \nu} B_{\mu \nu}\right]_{z=R},
$$

where $\kappa$ and $\kappa^{\prime}$ are dimensionless parameters. Then, for given values of $\left\{f_{\pi}, \kappa, \kappa^{\prime}\right\}$, we obtain with very good approximation

$$
g_{*} \approx \frac{e}{\sin \theta_{W}} \sqrt{\log \left(R^{\prime} / R\right)} \sqrt{1+\kappa^{2}}, \quad s_{\phi} \approx \tan \theta_{W} \sqrt{\frac{1+\kappa^{\prime 2}}{1+\kappa^{2}}} .
$$

Moreover,

$$
\sin \left(v / f_{\pi}\right) \approx 2 \frac{\sin \theta_{W}}{e} \frac{M_{W}}{f_{\pi}}
$$

and $e=\sqrt{4 \pi \alpha_{\mathrm{QED}}}$ is the electric charge while $\theta_{W}$ the Weinberg angle. In the dual CFT, changing $g_{*}$ corresponds to a change in the number of colors

$$
N_{\mathrm{CFT}} \equiv \frac{16 \pi^{2}}{g_{*}^{2}}
$$

The value of $g_{*}$ can also be related to the free parameter $g_{\rho}$ introduced in section 2 , controlling the mass scale of the composite vector resonances $m_{\rho} \equiv g_{\rho} f_{\pi}$. Considering the first Kaluza-Klein $(\mathrm{KK})$ resonance of a $5 \mathrm{D}$ gauge field with $(-,+)$ boundary conditions, ${ }^{23}$ we obtain

$$
g_{\rho} \approx 1.2024 \cdot g_{*} \cdot
$$

\footnotetext{
${ }^{23}$ In the holographic picture, these boundary conditions imply that this gauge field does not interact with the elementary sector.
} 
The fermion sector will depend on the $\mathrm{SO}(5)$ representation in which the $5 \mathrm{D}$ fields transform, $\mathbf{1}, \mathbf{4}, \mathbf{5}, \mathbf{1 0}$ or $\mathbf{1 4}$. Taking into account that $Y=T_{R}^{3}+X$ it is straightforward to work out all possible embeddings of the SM fermions. As mentioned in section 2, henceforth we will just consider fermions with a sizable degree of compositeness since they will be the only ones playing a non-negligible role in the generation of the Higgs potential and in the determination of the Higgs mass. That means in particular that we will neglect the first two quark generations, the right handed $(\mathrm{RH})$ bottom and, with the exception of the mMCHM ${ }^{\mathrm{III}}$ where all RH leptons will be composite, the first two lepton generations. Moreover, excepting again the mMCHM ${ }^{\mathrm{III}}$, possible $\mathrm{RH}$ neutrinos will also be neglected. In order to get the correct charged lepton masses we will still include left handed (LH) leptons when their RH counterparts are composite.

Due to the absence of $P_{L R}$ symmetry protecting the $Z \bar{b}_{L} b_{L}$ coupling, the spinorial representation is usually not considered for quarks as it would lead to too large deviations from its measured value. For leptons with a moderate degree of compositeness, we would encounter a similar problem for the corresponding $Z$ couplings. Thus, we will not consider this case throughout this work, restricting ourselves to the other representations. Without trying to exhaust all possible combinations of fermion representations, which have been discussed qualitatively in section 2, we will consider for the quark sector the cases where

- both $q_{L}$ and $t_{R}$ are embedded in a $\mathbf{5}, 10$ or 14, each, denoted by $\mathrm{MCHM}_{5}, \mathrm{MCHM}_{10}$ and $\mathrm{MCHM}_{14}$, respectively,

- the quark doublet $q_{L}$ is living in a $\mathbf{1 4}$ whereas the $t_{R}$ is a full singlet of $\mathrm{SO}(5)$, denoted by $\mathrm{MCHM}_{14-1}$,

- the quark doublet $q_{L}$ is embedded in a $\mathbf{5}$ and the $t_{R}$ in a $\mathbf{1 4}$, denoted by $\mathrm{MCHM}_{5-14}$.

Regarding leptons, the RH charged ones $e_{R}, \mu_{R}$ and $\tau_{R}$ will always be embedded in the $\mathbf{1 4}$, while the LH doublets will live either in the fundamental $\mathbf{5}$ or the symmetric representation 14.

In order to fix the notation and illustrate further the different embeddings we will describe in some detail the cases cited above. For further details on the $\mathrm{SO}(5)$ fermion representations we refer the reader to appendix B.

\subsection{1 $\mathrm{MCHM}_{5}$}

We consider two $\mathbf{5}_{\mathbf{2} / \mathbf{3}}$ of $\mathrm{SO}(5) \times \mathrm{U}(1)_{X}$ with the following boundary conditions

$$
\zeta_{1}=\left(\begin{array}{c}
\tilde{\Lambda}_{1}[-,+] t_{1}[+,+] \\
\tilde{t}_{1}[-,+] b_{1}[+,+]
\end{array}\right) \oplus t_{1}^{\prime}[-,+], \quad \zeta_{2}=\left(\begin{array}{c}
\tilde{\Lambda}_{2}[+,-] t_{2}[+,-] \\
\tilde{t}_{2}[+,-] b_{2}[+,-]
\end{array}\right) \oplus t_{2}^{\prime}[-,-],
$$

where we have explicitly shown the decomposition under $\mathrm{SU}(2)_{L} \times \mathrm{SU}(2)_{R}$, with the bidoublet being represented by a $2 \times 2$ matrix on which the $\mathrm{SU}(2)_{L}$ rotation acts vertically and the $\mathrm{SU}(2)_{R}$ one horizontally. More specifically, the left and right columns correspond to fields with $T_{R}^{3}= \pm 1 / 2$, whereas the upper and lower rows have $T_{L}^{3}= \pm 1 / 2$. The signs in square brackets denote the boundary conditions on the corresponding branes. A Dirichlet 
boundary condition for the $\mathrm{RH}$ chirality is denoted by $[+]$ while the opposite sign denotes the same boundary condition for the LH one. Therefore, before EWSB, zero-modes with quantum numbers $\mathbf{2}_{\mathbf{1} / \mathbf{6}}$ and $\mathbf{1}_{\mathbf{2} / \mathbf{3}}$ under $\mathrm{SU}(2)_{L} \times \mathrm{U}(1)_{Y}$ live in $\zeta_{1}$ and $\zeta_{2}$, respectively.

The relevant part of the action reads

$$
\mathcal{S} \supset \sum_{k=1,2} \int \mathrm{d}^{4} x \int_{R}^{R^{\prime}} \mathrm{d} z a^{4}\left\{\bar{\zeta}_{k}\left[i \not D+\left(D_{5}+2 \frac{a^{\prime}}{a}\right) \gamma^{5}-a M_{k}\right] \zeta_{k}\right\}+\mathcal{S}_{\mathrm{UV}}+\mathcal{S}_{\mathrm{IR}},
$$

where $^{24}$

$$
\begin{aligned}
D_{M}= & \partial_{M}-i g_{5} T_{L}^{a} L_{M}^{a}-i g_{5} T_{R}^{b} R_{M}^{b}-i g_{Y} Y B_{M}-i \frac{g_{Y}}{c_{\phi} s_{\phi}} Z_{M}^{\prime}\left(T_{R}^{3}-s_{\phi}^{2} Y\right) \\
& -i g_{5} T^{\hat{a}} C_{M}^{\hat{a}}, \quad \text { with } M=\mu, 5 \quad \text { and } \quad g_{Y} \equiv g_{5} g_{X} / \sqrt{g_{5}^{2}+g_{X}^{2}}
\end{aligned}
$$

are the gauge covariant derivatives and $\mathcal{S}_{\mathrm{UV}}, \mathcal{S}_{\mathrm{IR}}$ are possible brane localized terms. We conventionally parametrize the bulk masses $M_{k}=c_{k} / R$ in terms of dimensionless bulk mass parameters $c_{k}$ and the fundamental scale $R$.

In particular, the fifth component of the covariant derivative in the above action generates the Yukawa interactions

$$
\begin{aligned}
\mathcal{S} & \supset-\sum_{k=1,2} i g_{5} \int \mathrm{d}^{4} x \int_{R}^{R^{\prime}} \mathrm{d} z a^{4} \bar{\zeta}_{k}(x, z) \gamma^{5} T^{4} \zeta_{k}(x, z) C_{5}^{4}(x, z) \\
& =-i g_{5}\left[\int_{R}^{R^{\prime}} \mathrm{d} z a^{-1}\right]^{-1 / 2} \sum_{k=1,2} \int \mathrm{d}^{4} x \int_{R}^{R^{\prime}} \mathrm{d} z a^{3} \bar{\zeta}_{k}(x, z) \gamma^{5} T^{4} \zeta_{k}(x, z) h(x)+\ldots,
\end{aligned}
$$

where the dots stand for terms involving the non-physical KK excitations of the Higgs boson and we have used that the Higgs profile is given by

$$
f_{h}^{4}(z)=a^{-1}\left[\int_{R}^{R^{\prime}} \mathrm{d} z a^{-1}\right]^{-1 / 2}
$$

Looking at the specific form of the Yukawa interactions, we can see that in order to have a non-zero mass for the zero-modes after EWSB we need to have some IR brane terms splitting the zero-modes between the different multiplets. Therefore we consider the following brane action

$$
\mathcal{S}_{\mathrm{IR}}=-\int \mathrm{d}^{4} x\left\{a(z)^{4}\left[M_{S}^{q} \bar{\zeta}_{1 L}^{(\mathbf{1}, \mathbf{1})} \zeta_{2 R}^{(\mathbf{1 , 1})}+M_{B}^{q} \bar{\zeta}_{1 L}^{(\mathbf{2 , 2})} \zeta_{2 R}^{(\mathbf{2 , 2})}\right]\right\}_{z=R^{\prime}}+\text { h.c. }
$$

where we have used the decomposition $\zeta=\zeta^{(\mathbf{2 , 2})}+\zeta^{(\mathbf{1 , 1})}$ introduced explicitly in appendix B.1. One could in principle add some UV brane masses between the exotic $\mathrm{SU}(2)_{L}$ doublets of both bidoublets but their impact on the Higgs potential would be subleading, as they do not have zero-modes and the main contribution comes always from the elementary degrees of freedom. For the sake of simplicity, we will not consider the introduction

\footnotetext{
${ }^{24}$ See appendix A for explicit expressions of the $\mathrm{SO}(5)$ generators in the fundamental representation.
} 


\begin{tabular}{|c|c|}
\hline $5 \mathrm{D}$ & $4 \mathrm{D}$ \\
\hline KK mass & $\hat{m}_{T}, \tilde{m}_{T}$ \\
\hline$f_{c_{1},-c_{2}}$ & $y_{L, R}^{t} f_{\pi} / \hat{m}_{T} \approx \sin \varphi_{L, R}$ \\
\hline$M_{B}, M_{S}$ & $a_{L}, b_{R}$ \\
\hline fixed Higgs couplings $\left(C_{5}^{\hat{a}}\right)$ & $b_{L}, a_{R}$ \\
\hline
\end{tabular}

Table 1. Approximate correspondence between the parameters of the 5D model and those of the $4 \mathrm{D}$ model, introduced in section 2.

of fermionic brane kinetic terms unless they are required to get the correct EWSB as, for instance, in the case of the $\mathrm{MCHM}_{14-1}$, see below.

One remarkable feature of these models is that we can remove the Higgs vev $\left\langle h^{\hat{a}}\right\rangle=v \delta_{\hat{a} 4}$ from the bulk through the following $5 \mathrm{D}$ gauge transformation [61]

$$
\begin{aligned}
\zeta_{k}(x, z) & \rightarrow \Omega(z) \zeta_{k}(x, z), \\
\mathbb{A}_{M}(x, z) & \rightarrow \Omega(z) \mathbb{A}_{M}(x, z) \Omega(z)^{T}-\left(i / g_{5}\right)\left(\partial_{M} \Omega(z)\right) \Omega(z)^{T},
\end{aligned}
$$

where $\mathbb{A}_{M}=L_{M}^{a} T_{L}^{a}+R_{M}^{a} T_{R}^{a}+C_{M}^{\hat{a}} T^{\hat{a}}$ and

$$
\Omega(z)=\exp \left(-i g_{5} T^{\hat{a}}\left\langle h^{\hat{a}}(x)\right\rangle \int_{R}^{z} \mathrm{~d} z^{\prime} f_{h}^{\hat{a}}\left(z^{\prime}\right)\right)=\exp \left(-i g_{5} T^{4} v \int_{R}^{z} \mathrm{~d} z^{\prime} f_{h}^{4}\left(z^{\prime}\right)\right) .
$$

After such a transformation, we can solve the bulk equations of motion for fermions and gauge bosons neglecting the Higgs vev and impose the same UV boundary conditions, since $\Omega(R)=1$. However, the IR boundary conditions of the transformed fields will mix through the Wilson lines $\Omega\left(R^{\prime}\right)$ fields with the same electric charge.

Finally, similarly to the gauge sector, we can obtain the $4 \mathrm{D}$ parameter $g_{\Psi}$ introduced in the previous section by identifying the mass scale of the fermionic resonances in the $4 \mathrm{D}$ picture, $m_{\Psi} \equiv g_{\Psi} f_{\pi}$, with the mass of the first KK mode of a 5D field with boundary conditions $[-,+]$ and bulk mass $c_{\Psi}=1 / 2$. This leads, in the absence of fermion brane kinetic terms, to

$$
g_{\Psi}=g_{\rho} \approx 1.2024 \cdot g_{*}
$$

Although in this case $g_{\Psi}$ and $g_{\rho}$ turn out to be equal, this degeneracy could be lifted by adding fermion kinetic terms. For completeness we also show in table 1 the rest of approximate identifications with the $4 \mathrm{D}$ setup, where the function $f_{c}$

$$
f_{c} \equiv\left[\frac{1-2 c}{1-\left(\frac{R}{R^{\prime}}\right)^{1-2 c}}\right]^{\frac{1}{2}}
$$

gives the wave function of the corresponding fermion zero-mode on the IR brane. 


\subsection{2 $\mathrm{MCHM}_{10}$}

In this case we consider two $\mathbf{1 0}_{\mathbf{2} / \mathbf{3}}$ multiplets,

$$
\begin{gathered}
\zeta_{1}=\left(\begin{array}{c}
\tilde{\Lambda}_{1}[-,+] t_{1}[+,+] \\
\tilde{t}_{1}[-,+] b_{1}[+,+]
\end{array}\right) \oplus\left(\begin{array}{c}
\hat{\Lambda}_{1}[-,+] \\
\hat{t}_{1}[-,+] \\
\hat{b}_{1}[-,+]
\end{array}\right) \oplus\left(\begin{array}{c}
\Lambda_{1}^{\prime}[-,+] \\
t_{1}^{\prime}[-,+] \\
b_{1}^{\prime}[-,+]
\end{array}\right), \\
\zeta_{2}=\left(\begin{array}{c}
\tilde{\Lambda}_{2}[+,-] t_{2}[+,-] \\
\tilde{t}_{2}[+,-] b_{2}[+,-]
\end{array}\right) \oplus\left(\begin{array}{c}
\hat{\Lambda}_{2}[+,-] \\
\hat{t}_{2}[+,-] \\
\hat{b}_{2}[+,-]
\end{array}\right) \oplus\left(\begin{array}{c}
\Lambda_{2}^{\prime}[+,-] \\
t_{2}^{\prime}[-,-] \\
b_{2}^{\prime}[+,-]
\end{array}\right),
\end{gathered}
$$

where the first and second three-vectors in the $\mathrm{SO}(4)$ decomposition correspond to the $(\mathbf{3}, \mathbf{1})$ and $(\mathbf{1}, \mathbf{3})$ of $\mathrm{SO}(4)$, respectively. In the first case, the first, second and third components of the three-vector correspond to $T_{L}^{3}=+1,0,-1$, respectively, while the second case is analogous with the interchange $T_{L}^{3} \leftrightarrow T_{R}^{3}$. Similarly to the $\mathrm{MCHM}_{5}$, we consider the following brane action

$$
\begin{aligned}
\mathcal{S}_{\mathrm{IR}}= & -\int \mathrm{d}^{4} x\left\{a ( z ) ^ { 4 } \left[M_{T}^{q} \operatorname{Tr}\left(\bar{\zeta}_{1 L}^{(\mathbf{3}, \mathbf{1})} \zeta_{2 R}^{(\mathbf{3}, \mathbf{1})}+\bar{\zeta}_{1 L}^{(\mathbf{1}, \mathbf{3})} \zeta_{2 R}^{(\mathbf{1}, \mathbf{3})}\right)\right.\right. \\
& \left.\left.+M_{B}^{q} \operatorname{Tr}\left(\bar{\zeta}_{1 L}^{(\mathbf{2 , 2})} \zeta_{2 R}^{(\mathbf{2 , 2})}\right)\right]\right\}_{z=R^{\prime}}+\text { h.c. },
\end{aligned}
$$

where the decompositions $\zeta_{1,2}=\zeta_{1,2}^{(\mathbf{1 , 3})}+\zeta_{1,2}^{(\mathbf{3 , 1})}+\zeta_{1,2}^{(\mathbf{2 , 2})}$ are explicitly defined in appendix B.2.

\subsection{3 $\mathrm{MCHM}_{14}, \mathrm{MCHM}_{5-14}, \mathrm{MCHM}_{14-1}$}

MCHM $_{14}$. We will consider first the case of two $14_{2 / 3}$

$$
\begin{aligned}
& \zeta_{1}=t_{1}^{\prime}[-,+] \oplus\left(\begin{array}{c}
\tilde{\Lambda}_{1}[-,+] t_{1}[+,+] \\
\tilde{t}_{1}[-,+] b_{1}[+,+]
\end{array}\right) \oplus \zeta_{1}^{(\mathbf{3}, \mathbf{3})}[-,+], \\
& \zeta_{2}=t_{2}^{\prime}[-,-] \oplus\left(\begin{array}{c}
\tilde{\Lambda}_{2}[+,-] t_{2}[+,-] \\
\tilde{t}_{2}[+,-] b_{2}[+,-]
\end{array}\right) \oplus \zeta_{2}^{(\mathbf{3}, \mathbf{3})}[+,-],
\end{aligned}
$$

where this time, for the sake of simplicity, we have left implicit the components of the two $\mathrm{SO}(4)$ bi-triplets $\zeta_{1,2}^{(\mathbf{3 , 3})}$. The IR brane mass terms read

$$
\begin{aligned}
\mathcal{S}_{\mathrm{IR}}= & -\int \mathrm{d}^{4} x\left\{a ( z ) ^ { 4 } \left[M_{S}^{q} \operatorname{Tr}\left(\bar{\zeta}_{1 L}^{(\mathbf{1}, \mathbf{1})} \zeta_{2 R}^{(\mathbf{1}, \mathbf{1})}\right)+M_{B}^{q} \operatorname{Tr}\left(\bar{\zeta}_{1 L}^{(\mathbf{2}, \mathbf{2})} \zeta_{2 R}^{(\mathbf{2}, \mathbf{2})}\right)\right.\right. \\
& \left.\left.+M_{T}^{q} \operatorname{Tr}\left(\bar{\zeta}_{1 L}^{(\mathbf{3}, \mathbf{3})} \zeta_{2 R}^{(\mathbf{3}, \mathbf{3})}\right)\right]\right\}_{z=R^{\prime}}+\text { h.c. },
\end{aligned}
$$

where the explicit form of the fields $\zeta_{1,2}^{(\mathbf{1 , 1})}, \zeta_{1,2}^{(\mathbf{2 , 2})}$, and $\zeta_{1,2}^{(\mathbf{3 , 3})}$ can be obtained from appendix B.3.

Analogously to ref. [62] one can compute the contribution of the top quark and its tower of resonances to the $g g \rightarrow H$ loop induced process. This is particularly relevant for Higgs physics at the LHC and can impose some constraint on the composite parameters of the 
model, especially in this case where fields transform in $14 s$ of $\mathrm{SO}(5)$ and some sensitivity to the particular composite sector is expected $[18,44]$. To this end, we have computed the infinite sum $\sum_{n} v y_{n n}^{\text {top }} / m_{n}^{\text {top }}$, where $y_{n n}^{\text {top }}$ are the diagonal top-like Yukawa couplings and $m_{n}^{\text {top }}$ the corresponding (KK) masses, via the UV value of the full $5 \mathrm{D}$ propagator evaluated at zero-momentum, obtaining

$$
\begin{aligned}
\left.\sum_{n} \frac{v y_{n n}^{\text {top }}}{m_{n}^{\text {top }}}\right|_{\mathrm{MCHM}_{14}}= & \frac{\theta_{\pi}}{\cos \theta_{\pi} \sin \theta_{\pi}} \\
& \times \frac{3\left(M_{S}^{q}-M_{T}^{q}\right) \cos 2 \theta_{\pi}+\left(5 M_{S}^{q}-8 M_{B}^{q}+3 M_{T}^{q}\right) \cos 4 \theta_{\pi}}{3\left(M_{S}^{q}-M_{T}^{q}\right)+\left(5 M_{S}^{q}-8 M_{B}^{q}+3 M_{T}^{q}\right) \cos 2 \theta_{\pi}},
\end{aligned}
$$

where for the sake of simplicity we have introduced the shorthand notation $\theta_{\pi} \equiv v / f_{\pi}$. The corresponding cross-section normalized to the SM one is then given by

$$
\frac{\sigma(g g \rightarrow H)_{\mathrm{MCHM}_{14}}}{\sigma(g g \rightarrow H)_{\mathrm{SM}}} \approx \frac{\left|\kappa_{g}^{\mathrm{MCHM}_{14}}\right|^{2}+\left|\kappa_{g, 5}^{\mathrm{MCHM}_{14}}\right|^{2}}{\kappa_{v}^{2}},
$$

where $\kappa_{v}=v / v_{\mathrm{SM}}$ is the ratio of the $5 \mathrm{D}$ Higgs vev over the SM one and we have defined

$$
\kappa_{g}^{\mathrm{MCHM}_{14}}=\operatorname{Re}\left[\sum_{n} \frac{v y_{n n}^{\mathrm{top}}}{m_{n}^{\mathrm{top}}}\right]_{\mathrm{MCHM}_{14}}, \quad \kappa_{g, 5}^{\mathrm{MCHM}_{14}}=\frac{3}{2} \operatorname{Im}\left[\sum_{n} \frac{v y_{n n}^{\mathrm{top}}}{m_{n}^{\mathrm{top}}}\right]_{\mathrm{MCHM}_{14}} .
$$

To write (3.27) we have implicitly assumed $4 m_{\text {top }}^{2} / m_{H}^{2} \rightarrow \infty$ and neglected the subleading bottom contribution [44].

For the lepton case, we consider two $\mathbf{1 4}_{-\mathbf{1}}$ under $\mathrm{SO}(5) \times \mathrm{U}(1)_{X}$,

$$
\begin{aligned}
& \xi_{1}=\tau_{1}^{\prime}[-,+] \oplus\left(\begin{array}{c}
\nu_{1}[+,+] \tilde{\tau}_{1}[-,+] \\
\tau_{1}[+,+] \tilde{Y}_{1}[-,+]
\end{array}\right) \oplus \xi_{1}^{(\mathbf{3}, \mathbf{3})}[-,+],
\end{aligned}
$$

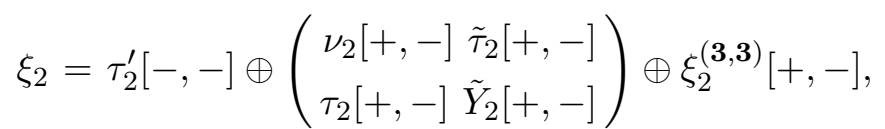

with an IR action analogous to the one of (3.25) with brane masses $M_{S}^{l}, M_{B}^{l}$ and $M_{T}^{l}$. We can also compute the analogous to (3.26) for the lepton case, which can be relevant for instance for $H \rightarrow \gamma \gamma$, obtaining the very same expression as in the quark case but with the change $\left\{M_{S}^{q}, M_{B}^{q}, M_{T}^{q}\right\} \rightarrow\left\{M_{S}^{l}, M_{B}^{l}, M_{T}^{l}\right\}$.

$\mathbf{M C H M}_{\mathbf{5 - 1 4}}$. This case can be obtained easily from the previous, changing the first $\mathbf{1 4}$ for a $\mathbf{5}$, i.e.,

$$
\begin{aligned}
& \zeta_{1}=t_{1}^{\prime}[-,+] \oplus\left(\begin{array}{c}
\tilde{\Lambda}_{1}[-,+] t_{1}[+,+] \\
\tilde{t}_{1}[-,+] b_{1}[+,+]
\end{array}\right), \\
& \zeta_{2}=t_{2}^{\prime}[-,-] \oplus\left(\begin{array}{c}
\tilde{\Lambda}_{2}[+,-] t_{2}[+,-] \\
\tilde{t}_{2}[+,-] b_{2}[+,-]
\end{array}\right) \oplus \zeta_{2}^{(\mathbf{3}, \mathbf{3})}[+,-],
\end{aligned}
$$


with the following IR action ${ }^{25}$

$$
\begin{aligned}
\mathcal{S}_{\mathrm{IR}}= & \int \mathrm{d}^{4} x \int_{R}^{R^{\prime}} \mathrm{d} z\left\{a^{4}(z)\left[\frac{\sqrt{5}}{2} M_{S}^{q}\left(\bar{\zeta}_{1 L}^{(\mathbf{1}, \mathbf{1})} \zeta_{2 R}^{(\mathbf{1}, \mathbf{1})}\right)_{5}+\sqrt{2} M_{B}^{q}\left(\bar{\zeta}_{1 L}^{(\mathbf{2}, \mathbf{2})} \zeta_{2 R}^{(\mathbf{2 , 2})}\right)_{5}\right]\right\}_{z=R^{\prime}} \\
& + \text { h.c.. }
\end{aligned}
$$

Similarly to the previous case, with two $\mathbf{1 4} s$, we can compute the top tower contribution to $g g \rightarrow H$, obtaining

$$
\begin{aligned}
& \sqrt{2} \theta_{\pi}\left\{\left[\sqrt { 1 0 } \operatorname { c o t } \theta _ { \pi } \left(\left(5 \cos 2 \theta_{\pi}+3\right) M_{S}^{q *}\left(\cos 2 \theta_{\pi}\left(5 \sqrt{2} M_{S}^{q}-4 \sqrt{5} M_{B}^{q}\right)-5 \sqrt{2} M_{S}^{q}\right)\right.\right.\right. \\
& \left.\left.-40 \cos ^{2} \theta_{\pi} M_{B}^{q *}\left(\cos 2 \theta_{\pi}\left(\sqrt{5} M_{S}^{q}-2 \sqrt{2} M_{B}^{q}\right)-\sqrt{5} M_{S}^{q}\right)\right)\right] \times \\
& {\left[\left(5 \cos 2 \theta_{\pi}+3\right) M_{S}^{q *}\left(5 \cos 2 \theta_{\pi}\left(\sqrt{10} M_{S}^{q}-4 M_{B}^{q}\right)-20 M_{B}^{q}+3 \sqrt{10} M_{S}^{q}\right)\right.} \\
& \left.-40 \cos ^{2} \theta_{\pi} M_{B}^{q *}\left(M_{S}^{q}\left(5 \cos 2 \theta_{\pi}+3\right)-4 \sqrt{10} M_{B}^{q} \cos ^{2} \theta_{\pi}\right)\right]^{-1} \\
& +\left[\left(5 \cos 2 \theta_{\pi}+3\right) M_{S}^{q *}\left(8 M_{S}^{q} \cot \theta_{\pi}-\sin 2 \theta_{\pi}\left(5 M_{S}^{q}-2 \sqrt{10} M_{B}^{q}\right)\right)\right. \\
& \left.-4 \cos ^{2} \theta_{\pi} M_{B}^{q *}\left(40 M_{B}^{q} \sin \theta_{\pi} \cos \theta_{\pi}+\sqrt{10} M_{S}^{q}\left(5 \cos 2 \theta_{\pi}+3\right) \cot \theta_{\pi}\right)\right] \times \\
& {\left[\left(5 \cos 2 \theta_{\pi}+3\right) M_{S}^{q *}\left(\sqrt{2} M_{S}^{q}\left(5 \cos 2 \theta_{\pi}+3\right)-8 \sqrt{5} M_{B}^{q} \cos ^{2} \theta_{\pi}\right)\right.} \\
& \left.\left.-8 \cos ^{2} \theta_{\pi} M_{B}^{q *}\left(\sqrt{5} M_{S}^{q}\left(5 \cos 2 \theta_{\pi}+3\right)-20 \sqrt{2} M_{B}^{q} \cos ^{2} \theta_{\pi}\right)\right]\right\}=\sum_{n} \frac{v y_{n n}^{\text {top }}}{\left.m_{n}^{\text {top }}\right|_{\mathrm{MCHM}_{5-14}}} .
\end{aligned}
$$

It is again straightforward to write down the lepton case, where we consider the following $\mathrm{SO}(5)$ multiplets,

$$
\begin{aligned}
& \xi_{1}=\tau_{1}^{\prime}[-,+] \oplus\left(\begin{array}{c}
\nu_{1}[+,+] \\
\tau_{1}[-,+] \\
\tau_{1}[+,+] \tilde{Y}_{1}[-,+]
\end{array}\right),
\end{aligned}
$$

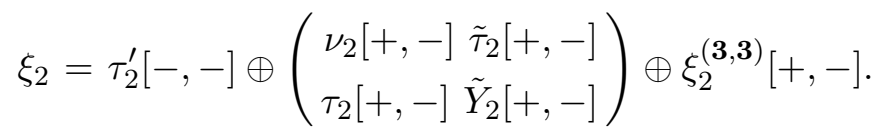

Analogous expressions to (3.33) and (3.34) hold in this case with the corresponding changes $\left\{M_{S}^{q}, M_{B}^{q}\right\} \rightarrow\left\{M_{S}^{l}, M_{B}^{l}\right\}$.

$\mathrm{MCHM}_{14-1}$. In this case the RH top is embedded in a full singlet of $\mathrm{SO}(5) \times \mathrm{U}(1)_{X}$, $\sim \mathbf{1}_{\mathbf{2} / \mathbf{3}}$, while the $\mathrm{SU}(2)_{L}$ doublet $q_{L}$ will live in $\mathbf{1 4}_{\mathbf{2} / \mathbf{3}}$,

$$
\begin{aligned}
& \zeta_{1}=t_{1}^{\prime}[-,+] \oplus\left(\begin{array}{c}
\tilde{\Lambda}_{1}[-,+] t_{1}[+,+] \\
\tilde{t}_{1}[-,+] b_{1}[+,+]
\end{array}\right) \oplus \zeta_{1}^{(\mathbf{3}, \mathbf{3})}[-,+], \\
& \zeta_{2}=t_{2}^{\prime}[-,-] .
\end{aligned}
$$

\footnotetext{
${ }^{25}$ For convenience, we have added prefactors $-\sqrt{5} / 2$ and $-\sqrt{2}$ to the boundary masses $M_{S}^{q}$ and $M_{B}^{q}$, respectively, in order to compensate group factors that will otherwise appear along with the brane masses mixing the canonically normalized fermions (making for instance the brane mass mixing the bidoublets slightly smaller than the one mixing the singlets).
} 
As was pointed out in [56], this setup has an accidental $\mathrm{SU}(9+4)=\mathrm{SU}(13)$ global symmetry on the IR brane (coming from the fact that the $\mathbf{9}_{\mathbf{2} / \mathbf{3}}=(\mathbf{3}, \mathbf{3})_{\mathbf{2} / \mathbf{3}}$ and the $\mathbf{4}_{\mathbf{2} / \mathbf{3}}=(\mathbf{2}, \mathbf{2})_{\mathbf{2} / \mathbf{3}}$ $\subset \mathbf{1 4}$ can be rotated as a single multiplet), leading in particular to a vanishing contribution to the $\sin ^{2}\left(h / f_{\pi}\right)$ coefficient of the Higgs effective potential. To break this symmetry, and be able to explore more general scenarios, we will introduce, also on the IR brane, fermion kinetic terms for both $\mathrm{SO}(4)$ multiplets, $\mathbf{4}_{\mathbf{2} / \mathbf{3}}$ and $\mathbf{9}_{\mathbf{2} / \mathbf{3}}$,

$$
\begin{aligned}
\mathcal{S}_{\mathrm{IR}}= & \int \mathrm{d}^{4} x\left\{a ( z ) ^ { 4 } \left[M_{S}^{q} \bar{\zeta}_{1 L}^{(\mathbf{1 , 1})} \zeta_{s 2 R}^{(\mathbf{1 , 1})}+\kappa_{B}^{q} R \operatorname{Tr}\left(\bar{\zeta}_{1 L}^{(\mathbf{2 , 2})} i \not \partial \zeta_{1 L}^{(\mathbf{2 , 2})}\right)\right.\right. \\
& \left.\left.+\kappa_{T}^{q} R \operatorname{Tr}\left(\bar{\zeta}_{1 L}^{(\mathbf{3}, \mathbf{3})} i \not \partial \zeta_{1 L}^{(\mathbf{3}, \mathbf{3})}\right)\right]\right\}_{z=R^{\prime}}+\text { h.c.. }
\end{aligned}
$$

In this particular case, the sensitivity to the composite parameters in $g g \rightarrow H$ disappears, getting

$$
\left.\sum_{n} \frac{v y_{n n}^{\text {top }}}{m_{n}^{\text {top }}}\right|_{\mathrm{MCHM}_{14-1}}=\theta_{\pi}\left(\cot \theta_{\pi}-\tan \theta_{\pi}\right) .
$$

\subsubsection{The mMCHM ${ }^{\mathrm{III}}$ : a new minimal model for leptons}

Taking a closer look to the structure of the symmetric representation of $\mathrm{SO}(5), \mathbf{1 4}=$ $(\mathbf{1}, \mathbf{1}) \oplus(\mathbf{2}, \mathbf{2}) \oplus(\mathbf{3}, \mathbf{3})$, one can readily see that it is the only one which can host at the same time a $P_{L R}$ protected $\mathrm{SU}(2)_{L} \times \mathrm{U}(1)_{Y}$ singlet and a triplet $\sim \mathbf{3}_{0}$. This feature implies in particular that, using this representation, we can build very minimal models in the lepton sector generating the neutrino masses through a type-III seesaw. In the following, we will consider the most minimal of these scenarios, which we have called mMCHM ${ }^{\mathrm{III}}$, realized with left- and right-handed leptons transforming as $\mathbf{5}_{-\mathbf{1}}$ and $\mathbf{1 4}_{-\mathbf{1}}$, respectively, under $\mathrm{SO}(5) \times \mathrm{U}(1)_{X}$ and the following boundary conditions

$$
\begin{aligned}
& \xi_{1 \tau}=\tau_{1}^{\prime}[-,+] \oplus\left(\begin{array}{cc}
\nu_{1}^{\tau}[+,+] & \tilde{\tau}_{1}[-,+] \\
\tau_{1}[+,+] & \tilde{Y}_{1}^{\tau}[-,+]
\end{array}\right)
\end{aligned}
$$

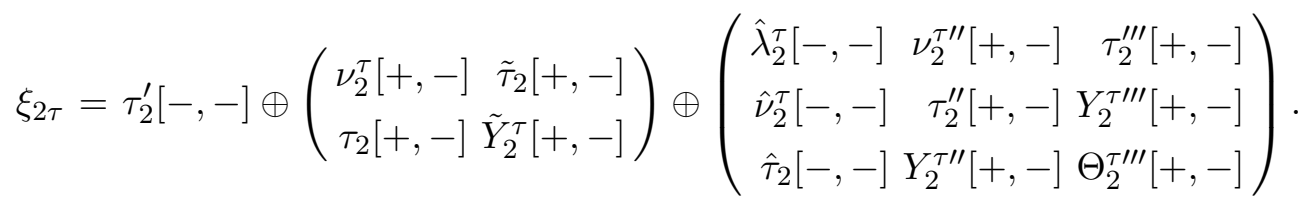

Here, we have just shown the two multiplets for the third generation, the ones for the first two generations are completely analogous. At the level of zero-modes we have the $\mathrm{SU}(2)_{L} \times \mathrm{U}(1)_{Y}$ spectrum $l_{\ell L}^{(0)} \sim \mathbf{2}_{-\mathbf{1} / \mathbf{2}} \subset \xi_{1 \ell}$ and $\ell_{R}^{(0)} \sim \mathbf{1}_{-\mathbf{1}}, \Sigma_{\ell R}^{(0)} \sim \mathbf{3}_{\mathbf{0}} \subset \xi_{2 \ell}$, with $\ell=$ $e, \mu, \tau$, which is the typical matter content for a $4 \mathrm{D}$ realization of the type-III seesaw [63].

In this case, we can write down the following UV Majorana mass term,

$$
\mathcal{S}_{\mathrm{UV}}=-\frac{1}{2} \sum_{\ell=e, \mu, \tau} \int \mathrm{d}^{4} x \int_{R}^{R^{\prime}} \mathrm{d} z\left\{a^{4}(z) M_{\Sigma}^{\ell} \operatorname{Tr}\left(\bar{\Sigma}_{\ell R} \Sigma_{\ell R}^{c}\right)\right\}_{z=R}+\text { h.c. }
$$

where

$$
\Sigma_{\ell}=\left(\begin{array}{cc}
\hat{\nu}_{2}^{\ell} / \sqrt{2} & \hat{\lambda}_{2}^{\ell} \\
\ell_{2} & -\hat{\nu}_{2}^{\ell} / \sqrt{2}
\end{array}\right), \quad \ell=e, \mu, \tau
$$


are the $5 \mathrm{D} \mathrm{SU}(2)_{L} \times \mathrm{U}(1)_{Y}$ triplets hosting the previously introduced $\Sigma_{\ell R}^{(0)}$ zero-modes. On the other hand, the IR brane masses read

$$
\mathcal{S}_{\mathrm{IR}}=\sum_{\ell=e, \mu, \tau} \int \mathrm{d}^{4} x\left\{a(z)^{4}\left[\frac{\sqrt{5}}{2} M_{S}^{\ell}\left(\bar{\xi}_{1 \ell L}^{(\mathbf{1}, \mathbf{1})} \xi_{2 \ell R}^{(\mathbf{1}, \mathbf{1})}\right)_{5}+\sqrt{2} M_{B}^{\ell}\left(\bar{\xi}_{1 \ell L}^{(\mathbf{2}, \mathbf{2})} \xi_{2 j R}^{(\mathbf{2 , 2})}\right)_{5}\right]\right\}_{z=R^{\prime}}+\text { h.c. }
$$

where, for the sake of simplicity, as we are just interested at the moment in the size of the contribution to the Higgs potential, we have assumed that all brane masses $M_{\Sigma}^{\ell}, M_{S}^{\ell}$ and $M_{B}^{\ell}$ are flavor diagonal.

The underlying reason for considering all three generations in this case rather than just the third one is related to the size of the effective Majorana mass, i.e., the Majorana mass for the corresponding zero-modes

$$
\mathcal{M}_{\mathrm{M}}^{\ell \ell^{\prime}} \approx \frac{f_{-c_{2}^{\ell}}^{2}}{R^{\prime}}\left(\frac{R^{\prime}}{R}\right)^{-2 c_{2}^{\ell^{\prime}}} M_{\Sigma}^{\ell} \delta_{\ell \ell^{\prime}}, \quad \ell, \ell^{\prime} \in\{e, \mu, \tau\}
$$

This mass is typically too large unless the corresponding zero-mode profiles are pushed away from the UV brane. This leads to values of $c_{2}^{\ell} \in(-1 / 2,0)$ and thus IR localized RH zero-modes. Therefore, we can see that in this scenario, just the quantum numbers of the lepton sector and the overall scale of the neutrino masses lead naturally to IR localized leptons for all three generations. As we will see below, this will allow us to compensate the relative color suppression of the lepton sector in the contribution to the Higgs potential, making this setup particularly interesting for lifting the masses of the top partners.

\subsection{The one-loop Higgs mass}

We now move forward to derive the Higgs potential and in particular the Higgs mass in the explicit 5D models. After some discussion on the matching to $m_{H}$, we review the calculation of the Coleman-Weinberg Potential in extra dimensional theories and finally present explicit results for the models studied in this work.

\subsubsection{The Higgs mass at $f_{\pi}$}

In the following, we want to estimate the value of the Higgs mass that should be matched to in the calculation of the 1-loop Higgs potential at the scale $f_{\pi}$ for the models under consideration. Neglecting higher dimensional operators with $D \geq 6$, whose effect should be suppressed, the potential can be written as

$$
V(h)=\alpha \sin ^{2}\left(h / f_{\pi}\right)-\beta \sin ^{2}\left(h / f_{\pi}\right) \cos ^{2}\left(h / f_{\pi}\right) \simeq \mu^{2} h^{2}+\lambda h^{4}+\mathcal{O}\left(h^{6}\right),
$$

where $\mu$ and $\lambda$ are functions of $\alpha$ and $\beta$. Now these coefficients are fixed by the Higgs mass $m_{H}$ and the Higgs vev $v$. In particular,

$$
\lambda=m_{H}^{2} /\left(8 v^{2}\right),
$$

which means that we can use this standard relation to obtain the running of the Higgs mass from the running of the quartic as $m_{H}(\mu)=\sqrt{8 \lambda(\mu)} v$. 
Along the same lines, the running of $\lambda$ should be given to first approximation by the running of the $D \leq 4$ part of the Lagrangian, i.e., the SM. ${ }^{26}$ Setting $f_{\pi} \sim 1 \mathrm{TeV}$ and employing the SM result (see e.g. [64])

$$
\frac{\lambda(1 \mathrm{TeV})}{\lambda\left(m_{H}=125 \mathrm{GeV}\right)} \approx 0.7
$$

we finally arrive at $\mathrm{a} \sim 20 \%$ correction

$$
m_{H}\left(f_{\pi} \sim 1 \mathrm{TeV}\right) \approx \sqrt{\frac{\lambda(1 \mathrm{TeV})}{\lambda\left(m_{H}=125 \mathrm{GeV}\right)}} m_{H} \approx 105 \mathrm{GeV}
$$

Motivated by this result, we will employ $m_{H}\left(f_{\pi}\right)=105 \mathrm{GeV}(1 \pm 7.5 \%)$ in the following numerical analysis, accounting for the uncertainties in the running in a conservative way. Thus, the top mass and the Higgs mass are treated on the same footing, evaluated at $f_{\pi}$.

\subsubsection{Coleman-Weinberg potential in KK theories}

The contribution to the one-loop Coleman-Weinberg potential of a particular KK tower can be written as follows

$$
V(h) \supset \frac{N}{2} \sum_{n=1}^{\infty} \int \frac{\mathrm{d}^{4} p}{(2 \pi)^{4}} \log \left(p^{2}+m_{n}^{2}(h)\right),
$$

where $N$ is the number of degrees of freedom of the corresponding type of resonance $N=+3$ for gauge bosons and $N=-4$ for fermions - and $m_{n}(h)$, with $n \in \mathbb{N}$, are their masses in the presence of the Higgs background. It can be shown, see e.g. [49, 65], that the previous infinite sum can be exchanged by an integral on the Minkowski space, leading to

$$
V(h)=\sum_{r} \frac{N_{r}}{(4 \pi)^{2}} \int_{0}^{\infty} \mathrm{d} p p^{3} \log \rho_{r}\left(-p^{2}\right)
$$

where $r$ sums over the different KK towers of the model and $\rho_{r}\left(w^{2}\right), w \in \mathbb{C}$, are some spectral functions, holomorphic in the $\operatorname{Re}(w)>0$ part of the complex plane and with roots in the real axis encoding the physical spectrum, i.e.,

$$
\rho_{r}\left(m_{n ; r}^{2}(h)\right)=0, \quad n \in \mathbb{N} .
$$

In models of GHU, where the Higgs background can be removed except for one brane through the transformations (3.16) and (3.17), it is actually possible to compute these spectral functions and therefore to have a calculable and finite Higgs potential, as these functions will go to zero fast enough for large values of $p^{2}$. We will focus on them in the following subsection.

\footnotetext{
${ }^{26}$ Although, approaching the scale $\mu=f_{\pi}$ the renormalization group evolution will receive modifications, most of the effect will be due to the SM, for $f_{\pi} \gg m_{H}$.
} 


\subsubsection{The Higgs mass in the MCHMs}

We will consider first the contribution of the gauge boson sector, which is model independent for our choice of symmetry breaking $\mathrm{SO}(5) \rightarrow \mathrm{SO}(4)$. Then we will study the cases of the different fermion representations considered in this work. We would like to remark that the corresponding form factors will include obviously in all cases subleading effects of the full mixing with the heavy resonances.

Gauge bosons. The form factors for gauge bosons are well known in the literature [5]. With our notation and the UV brane kinetic terms of (3.5) they read

$$
\rho_{Z, W}\left(p^{2}\right)=1+f_{Z, W}\left(p^{2}\right) \sin ^{2}\left(h / f_{\pi}\right),
$$

with

$$
\begin{aligned}
f_{Z}\left(p^{2}\right)= & \frac{p}{2}\left(\frac{R^{\prime}}{R}\right)\left[\left(1+s_{\phi}^{2}\right) C^{\prime}\left(p, R^{\prime}\right)-p R \log \left(R^{\prime} / R\right)\left(\kappa^{\prime 2}+\kappa^{2} s_{\phi}^{2}\right) S^{\prime}\left(p, R^{\prime}\right)\right] \times \\
& \times\left[S\left(p, R^{\prime}\right)\left(C^{\prime}\left(p, R^{\prime}\right)-p R \log \left(R^{\prime} / R\right) \kappa^{2} S^{\prime}\left(p, R^{\prime}\right)\right)\right. \\
f_{W}\left(p^{2}\right)= & \frac{p}{2}\left(\frac{R^{\prime}}{R}\right) \frac{\left.\left(C^{\prime}\left(p, R^{\prime}\right)-p R \log \left(R^{\prime} / R\right) \kappa^{\prime 2} S^{\prime}\left(p, R^{\prime}\right)\right)\right]^{-1}}{S\left(p, R^{\prime}\right)\left(C^{\prime}\left(p, R^{\prime}\right)-p R \log \left(R^{\prime} / R\right) \kappa^{2} S^{\prime}\left(p, R^{\prime}\right)\right)} .
\end{aligned}
$$

In the expressions above, $C(m, z)$ and $S(m, z)$ are functions satisfying the bulk equations of motion for gauge bosons and a vanishing Higgs vev, with boundary conditions $C(m, R)=$ $1, C^{\prime}(m, R)=0, S(m, R)=0, S^{\prime}(m, R)=m$, where the prime denotes a derivative with respect to the extra-dimensional coordinate. They are given by

$$
\begin{aligned}
C(m, z) & =\frac{\pi}{2} m z\left[Y_{0}(m R) J_{1}(m z)-J_{0}(m R) Y_{1}(m z)\right], \\
S(m, z) & =\frac{\pi}{2} m z\left[Y_{1}(m z) J_{1}(m R)-J_{1}(m z) Y_{1}(m R)\right] .
\end{aligned}
$$

We obtain

$$
\begin{aligned}
V_{g}(h) & =\frac{3}{(4 \pi)^{2}} \int_{0}^{\infty} \mathrm{d} p p^{3}\left[\log \rho_{Z}\left(-p^{2}\right)+2 \log \rho_{W}\left(-p^{2}\right)\right] \\
& =\frac{3}{32 \pi^{2}} \int_{0}^{\infty} \mathrm{d} t t\left[\log \rho_{Z}(-t)+2 \log \rho_{W}(-t)\right],
\end{aligned}
$$

after performing the change of variables $t=p^{2}$. The above potential can be written to very good approximation as

$$
V(h) \approx \alpha_{g} \sin ^{2}\left(h / f_{\pi}\right)-\beta_{g} \sin ^{2}\left(h / f_{\pi}\right) \cos ^{2}\left(h / f_{\pi}\right),
$$

where we have defined

$$
\begin{aligned}
\alpha_{g} & =\frac{3}{32 \pi^{2}} \int_{\mu_{\mathrm{IR}}}^{\infty} \mathrm{d} t t\left\{\left[f_{Z}(-t)-\frac{1}{2}\left(f_{Z}(-t)\right)^{2}\right]+2\left[f_{W}(-t)-\frac{1}{2}\left(f_{W}(-t)\right)^{2}\right]\right\}, \\
\beta_{g} & =-\frac{3}{64 \pi^{2}} \int_{\mu_{\mathrm{IR}}}^{\infty} \mathrm{d} t t\left\{\left(f_{Z}(-t)\right)^{2}+2\left(f_{W}(-t)\right)^{2}\right\},
\end{aligned}
$$

and introduced an IR regulator $\mu_{\mathrm{IR}}$ in order to cure the spurious IR divergences arising from the former expansion [5, 11]. Anyway, for the calculations perfomed in this work we just used the exact expression (3.59) as well as its analogues for the fermion case. 
Fermions. Although the case of fermions is very model dependent, we can still perform some general discussion. Contrary to the gauge boson form factors, in the fermion case we will have in general further powers of $\sin ^{2}\left(h / f_{\pi}\right)$. In particular, for all the scenarios we consider in this work

$$
\begin{aligned}
\rho_{f}\left(p^{2}\right)= & 1+f_{2}^{f}\left(p^{2}\right) \sin ^{2}\left(h / f_{\pi}\right)+f_{4}^{f}\left(p^{2}\right) \sin ^{4}\left(h / f_{\pi}\right) \\
& +f_{6}^{f}\left(p^{2}\right) \sin ^{6}\left(h / f_{\pi}\right)+f_{8}^{f}\left(p^{2}\right) \sin ^{8}\left(h / f_{\pi}\right), \quad f \in\{t, e, \mu, \tau\},
\end{aligned}
$$

where some of the functions $f_{k}^{f}\left(p^{2}\right)$, with $k \in\{2,4,6,8\}$, may be identically zero. We have computed all of them in a similar way to the previous case but their explicit expressions are too involved to justify the inclusion in this work. The corresponding contributions to the Higgs potential read

$$
V_{f}(h)=-\frac{4}{32 \pi^{2}} N_{f} \int_{0}^{\infty} \mathrm{d} t t \log \rho_{f}(-t),
$$

where $N_{f}$ is a possible color factor, i.e., $N_{t}=3$ and $N_{e, \mu, \tau}=1$. Again, one could use with very good approximation

$$
V(h) \approx \alpha_{f} \sin ^{2}\left(h / f_{\pi}\right)-\beta_{f} \sin ^{2}\left(h / f_{\pi}\right) \cos ^{2}\left(h / f_{\pi}\right),
$$

where we have neglected terms of $\mathcal{O}\left(\sin ^{6}\left(h / f_{\pi}\right)\right)$ and $\mathcal{O}\left(\sin ^{8}\left(h / f_{\pi}\right)\right)$ and defined

$$
\begin{aligned}
& \alpha_{f}=-\frac{4}{32 \pi^{2}} N_{f} \int_{\mu_{\mathrm{IR}}}^{\infty} \mathrm{d} t t\left[f_{2}^{f}(-t)+f_{4}^{f}(-t)-\frac{1}{2}\left(f_{2}^{f}(-t)\right)^{2}\right], \\
& \beta_{f}=-\frac{4}{32 \pi^{2}} N_{f} \int_{\mu_{\mathrm{IR}}}^{\infty} \mathrm{d} t t\left[f_{4}^{f}(-t)-\frac{1}{2}\left(f_{2}^{f}(-t)\right)^{2}\right] .
\end{aligned}
$$

However, as already mentioned, in practice we will just consider the exact expression in equation (3.63).

\subsection{Lifting light partners with leptons: numerical results and discussion}

In order to be able to quantify the effect of leptons on the Higgs mass and the scale of the top partners we have performed a numerical scan focusing on two main scenarios considered in this work, namely, non-minimal models of composite leptons arising from a flavor protection mechanism featuring a composite $\tau_{R}$, and minimal models of type-III seesaw where brane masses can in principle be large and all three lepton generations have an impact. In both cases, we consider $R=10^{-16} \mathrm{TeV}^{-1}$ and fixed values of $f_{\pi} \lesssim 1 \mathrm{TeV}$, as well as $\kappa=\kappa^{\prime}=0$, which correspond to $g_{*} \approx 4.0$ and $s_{\phi} \approx \tan \theta_{W}$. We also scan uniformly $\kappa, \kappa^{\prime} \in[0, \sqrt{3}]$, which translates to $g_{*} \sim[4 ., 8$.$] (or, equivalently, N_{\mathrm{CFT}} \sim[2.5,9.8]$ ) and $s_{\phi} \sim$ $\left[1 / 2 \tan \theta_{W}, 2 \tan \theta_{W}\right]$. Regarding the fermion sector, we have assumed random complex brane masses

$$
\left|M_{S}^{q}\right|,\left|M_{B}^{q}\right|,\left|M_{T}^{q}\right| \leq Y_{*}^{q}, \quad\left|M_{S}^{l}\right|,\left|M_{B}^{l}\right|,\left|M_{T}^{l}\right| \leq Y_{*}^{l}, \quad M_{S}^{q, l}, M_{B}^{q, l}, M_{T}^{q, l} \in \mathbb{C},
$$

in the first case, while we have taken real brane lepton masses in the second one for simplicity,

$$
\left|M_{\Sigma}^{\ell}\right|,\left|M_{S}^{\ell}\right|,\left|M_{B}^{\ell}\right| \leq Y_{*}^{l}, \quad M_{\Sigma}^{\ell}, M_{S}^{\ell}, M_{B}^{\ell} \in \mathbb{R}, \quad \text { with } \quad \ell=e, \mu, \tau,
$$


(with the quark brane masses analogous to (3.67)) as the introduction of the Majorana masses $M_{\Sigma}^{\ell}$ would otherwise double the size of the system of equations that we have to solve. ${ }^{27}$ The numbers $Y_{*}^{q}, Y_{*}^{l} \in \mathbb{R}^{+}$are fixed to some benchmark values specified below. The quark bulk masses $c_{1}^{q}$ and $c_{2}^{q}$ were fixed in both cases requiring

$$
\left.\frac{\partial V(h)}{\partial h}\right|_{h=v}=0, \quad m_{t}=m_{t}^{\mathrm{ref}}
$$

with $^{28}$

$$
V(h)=V_{g}(h)+\sum_{f} V_{f}(h), \quad m_{t}^{\mathrm{ref}} \in[145,155] \mathrm{GeV},
$$

while the procedure of fixing the lepton bulk masses in each case differs. In the scenario of $\tau_{R}$ compositeness, where the neutrino sector is left unspecified and the only constraint is $m_{\tau}=1.7 \mathrm{GeV}$, we randomly scan over $c_{2}^{\tau} \in[-0.5,0.5]$, fixing the other bulk mass via the $\tau$ mass. However, when considering minimal type-III seesaw models, we fix for each generation both bulk masses from the corresponding charged lepton and neutrino masses, imposing for the latter

$$
m_{\nu}^{\ell}=m_{\nu}^{\ell ; \text { ref }}, \quad \text { with } \quad m_{\nu}^{\ell ; \text { ref }}=\varepsilon_{\ell} 10^{-p_{\ell}} \mathrm{eV} \quad \text { and } \quad \varepsilon_{\ell} \in[0,1], p_{\ell} \in[0,3] .
$$

We have ensured that all points in our random scan are in reasonable agreement with electroweak precision data by choosing $f_{\pi}=0.8$ and $1.0 \mathrm{TeV}$, since

$$
T=0, \quad S \approx \frac{3}{2} \pi v^{2} R^{\prime 2}=6 \pi\left(\frac{v}{f_{\pi}}\right)^{2} g_{*}^{-2}, \quad U=0,
$$

in these models at tree level $[5,11,24]$ and $f_{\pi}=0.8(1.0) \mathrm{TeV}$ implies in particular

$$
S \in[0.028(0.018), 0.112(0.071)] .
$$

In general, although $\left.S\right|_{U=0}=0.05 \pm 0.09$ at $95 \%$ confidence level [66], the correlation with the predicted value of the $T$ parameter is large, $\rho_{\text {corr. }}=0.91$, which would in principle disfavor a large portion of the values above. We should however keep in mind the limitations of this analysis, as we are neglecting non-oblique effects as well as radiative fermion corrections which can give an non-negligible positive contribution to the $T$ parameter [16]. In addition to this, we have also checked that $\left|\delta g_{Z \bar{\ell} \ell} / g_{Z \bar{\ell} \ell}\right| \leq 2 \%$, with $\ell=e, \mu, \tau$, for all points on the scan.

Finally, before going to the specific results, let us briefly comment on how we measured the fine-tuning of the models at hand. Although a full examination of the tuning of these models including all possible brane kinetic terms is well beyond the scope of this paper, see e.g [11] for the case of the $\mathrm{MCHM}_{5}$, we still wanted to have a qualitative idea of the amount of tuning required by these setups, as well as being able to compare them with

\footnotetext{
${ }^{27}$ Which is already $10 \times 10$ in this case.

${ }^{28}$ Remember that $v$ was fixed from $\left\{\alpha_{\mathrm{QED}}, M_{W}, M_{Z}\right\}$ together with $g_{*}$ and $s_{\phi}$.
} 
other scenarios lifting the masses of the top partners. To this end, we have computed the Barbieri-Giudice measure of the tuning [67], defined as

$$
\Delta_{\mathrm{BG}}=\max _{i, j}\left|\frac{\partial \log \mathcal{O}_{i}}{\partial X_{j}}\right|,
$$

where $\mathcal{O}_{i}$ are the observables considered and $X_{j}$ the different inputs of the model. In particular, for every point of our numerical scan, we have computed the derivatives of the logarithm of both the $W$ and the Higgs mass ${ }^{29}$ imposing EWSB (i.e., $\partial V(h) /\left.\partial h\right|_{h=v}=0$ ) with respect of all variables of the model and taken the maximum.

Before checking explicitly the impact of leptons in both scenarios, let us first review the behavior of the different quark representations that we are considering.

\subsubsection{Minimal quark setups}

We start by studying the $\mathrm{MCHM}_{5}$ and $\mathrm{MCHM}_{10}$ as two representatives of models mixing the top quarks with fundamental and adjoint representations of $\mathrm{SO}(5)$. Here, only the fact that both $t_{L}$ and $t_{R}$ mix with a $\mathbf{5}$ or a $\mathbf{1 0}$ is important, while the embedding of the composites that mix with the lighter quarks is irrelevant to excellent approximation. Also, as discussed in section 2.2, setups of this class that mix the left handed top with another representations than the right handed top do not show a qualitatively new behavior. In consequence, these benchmark models exploit the full range of predictions of setups mixing each top chirality with a representation smaller than the symmetric one. For the time being, the contribution of the leptons will be rendered irrelevant by embedding them in single fundamental or adjoint representations and/or assuming a negligible lepton compositeness.

In figure 1 a we show the mass of the lightest $Q=2 / 3$ resonance, $m_{2 / 3}^{\min }$, as a function of the Higgs mass evaluated at $f_{\pi}$ for the $\mathrm{MCHM}_{5}$, considering two values for the maximally allowed brane masses, $Y_{*}^{q}=0.7$ and $Y_{*}^{q}=1.4 .{ }^{30}$ We also give the dependence on the bulk mass of the $\mathrm{SO}(5)$ multiplet hosting mostly the right-handed top, $c_{2}^{q}$ (where $f_{-c_{2}}$ corresponds to $y_{R}^{t} f_{\pi} / \tilde{m}_{T} \approx \sin \varphi_{R}^{t}$ in the $4 \mathrm{D}$ setup, see table 1 ). The darker the hue of the blue points, the smaller $c_{2}^{q}$. Finally, note that we set $f_{\pi}=0.8 \mathrm{TeV}$ and assume no brane localized kinetic terms for the main part of the analysis. We will comment on their impact at the end of this subsection. The plots confirm the discussion of section 2.2. First, the general scale of the predicted Higgs mass is as low as $m_{H} \lesssim v$, in agreement with the fact that $\beta_{t}$ is only generated at subleading order in the spurions, see (2.24). Moreover, we see clearly that the smaller the mass of the Higgs boson, the lighter the top partners need to be, confirming (2.25). In particular, the light Higgs with mass $m_{H}\left(f_{\pi}\right)=105 \mathrm{GeV}(1 \pm 7.5 \%)$, evaluated at $f_{\pi}$ (see section 3.2.1) and depicted by the yellow band, requires top partners below a TeV. We can also see that, as expected from table 1, the lighter the partners, the bigger in general the values of $c_{2}^{q}$, which in the $5 \mathrm{D}$ setup corresponds to a larger mixing with

\footnotetext{
${ }^{29}$ Note that the condition $\partial V(h) /\left.\partial h\right|_{h=v}=0$ defines $v$ and thus the $\mathrm{W}$ mass as a function of all other variables.

${ }^{30}$ Here, $m_{2 / 3}^{\min }$ corresponds to $\min \left(m_{T}, m_{\tilde{T}}\right)$ in $(2.25)$. Moreover, since every $\mathrm{SU}(2)_{L}$ representation present in the setups we consider features a $Q=2 / 3$ entry, $m_{2 / 3}^{\min }$ also corresponds to good approximation to the mass of the lightest mode of the whole spectrum of resonances.
} 

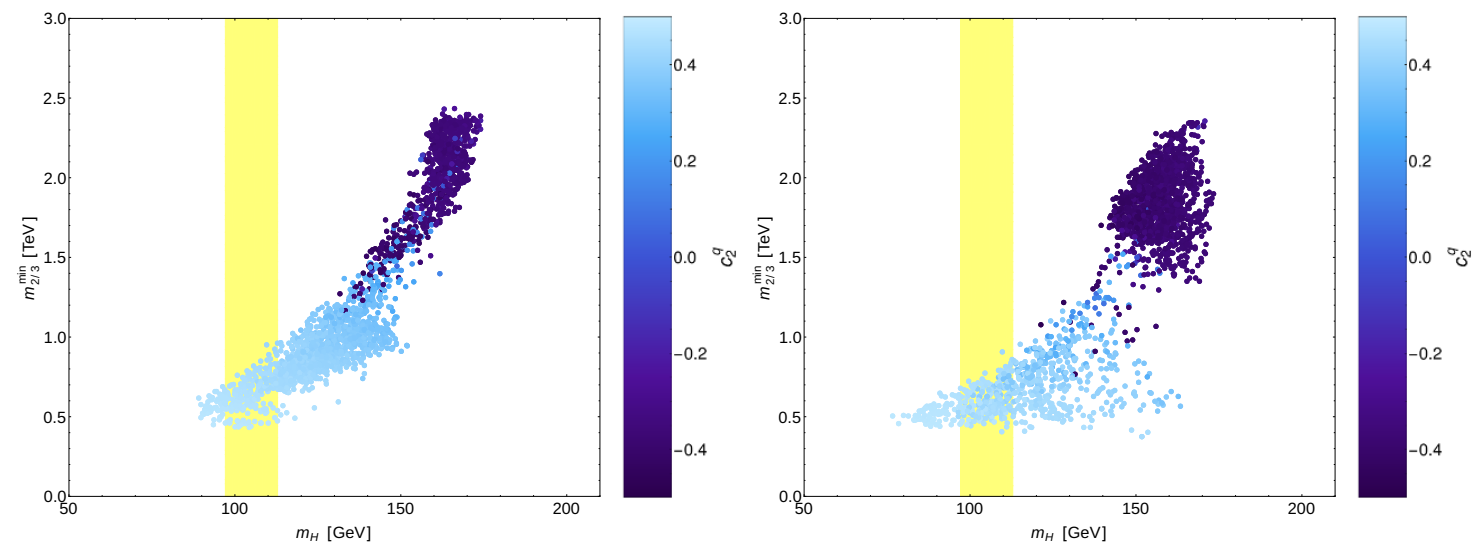

(a) $Y_{*}^{q}=0.7$ (left plot) and $Y_{*}^{q}=1.4$ (right plot) in the $\mathrm{MCHM}_{5}$ with $f_{\pi}=0.8 \mathrm{TeV}$ and $\kappa=\kappa^{\prime}=0$.
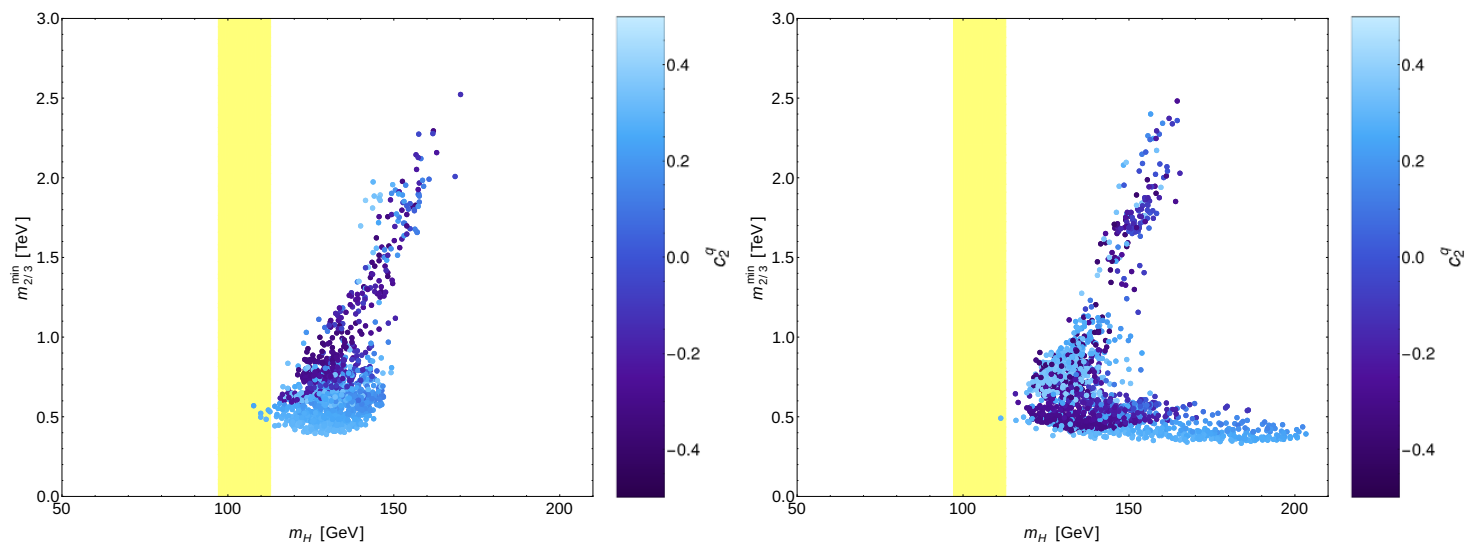

(b) $Y_{*}^{q}=0.7$ (left plot) and $Y_{*}^{q}=1.4$ (right plot) in the $\mathrm{MCHM}_{10}$ with $f_{\pi}=0.8 \mathrm{TeV}$ and $\kappa=\kappa^{\prime}=0$.

Figure 1. Mass of the first top partner as a function of the Higgs mass in the $\mathrm{MCHM}_{5}$ and $\mathrm{MCHM}_{10}$, figures $1 \mathrm{a}$ and $1 \mathrm{~b}$. In both cases, we have fixed $f_{\pi}=0.8 \mathrm{TeV}$ and $\kappa=\kappa^{\prime}=0$, corresponding roughly to $g_{*} \sim 4$ or $N_{\mathrm{CFT}} \sim 10$, and $s_{\phi} \approx \tan \theta_{W}$. Lighter points correspond to more IR localized $t_{R}$. The yellow band corresponds to a $\pm 7.5 \%$ variation around $m_{H}\left(f_{\pi}\right)=105 \mathrm{GeV}$.

the composite sector due to the stronger IR localization. On the other hand, there exist gradients in the $m_{H}-m_{2 / 3}^{\min }$ plane with constant $c_{2}^{q}$, where the larger Goldstone-symmetry breaking due to a larger $y_{R}^{t}$ is coming with an increased partner mass such as to mostly cancel the effect on $c_{2}^{q}$. The analogous plots for the $\mathrm{MCHM}_{10}$ are shown in figure $1 \mathrm{~b}$. While the qualitative behavior is similar, quantitatively the situation is more severe in this model where also the $b_{L}$ contributes to the potential, since the top partners need to be even lighter to arrive at the correct Higgs mass. The required $m_{2 / 3}^{\min } \lesssim(500-600) \mathrm{GeV}$ is already in conflict with LHC searches. Moreover, the experimental Higgs mass is at the boundary of the viable parameter space of the model. For the $\mathrm{MCHM}_{5}$, this is quantified further in figure 2, where we show the fraction of parameter points $P_{m}$ out of all points that reproduce the correct Higgs mass $m_{H}\left(f_{\pi}\right)=105 \mathrm{GeV}(1 \pm 7.5 \%)$, for which the lightest top partner is heavier than $m_{2 / 3}^{\min }$, versus $m_{2 / 3}^{\min }$, assuming $f_{\pi}=0.8 \mathrm{TeV}$. The curve has been obtained by smoothening the corresponding histograms and the black line depicts the $95 \%$ 


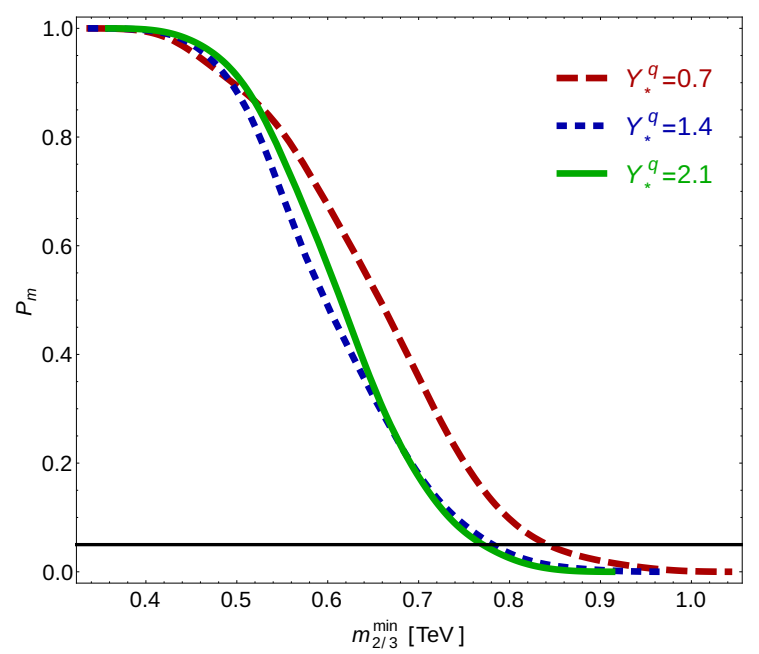

Figure 2. Survival function $\mathcal{P}_{m}$ of the first top partner mass in the $\mathrm{MCHM}_{5}$, for $f_{\pi}=0.8 \mathrm{TeV}$, $\kappa=\kappa^{\prime}=0$, and $Y_{*}^{q} \in\{0.7,1.4,2.1\}$, assuming $m_{H}\left(f_{\pi}\right)=105 \mathrm{GeV}(1 \pm 7.5 \%)$.

quantile. For all brane masses considered, we find that $\gtrsim 90 \%$ of the points feature a top partner below $800 \mathrm{GeV}$, in good agreement with (2.26). The experimental bounds already cut significantly into the parameter space of the model [55]. As in the $\mathrm{MCHM}_{10}$ the viable $m_{H}$ region is hardly reachable, we do not show the corresponding plot for that model. It is evident that the Higgs mass (in particular together with the absence of light partners) already practically rules out this model. If the LHC excludes $Q=2 / 3$ partners up to a TeV, which is possible with less than $300 \mathrm{fb}^{-1}$ of data at the LHC14 $[68,69]$, also the $\mathrm{MCHM}_{5}$ would be excluded for practical purposes as a full solutions to the hierarchy problem.

Now we turn to the tuning present in the models, which we show in figures 3 and 4 for $f_{\pi}=0.8 \mathrm{TeV}$ and $f_{\pi}=1 \mathrm{TeV}$, respectively. As explained before, we describe the tuning by the Barbieri-Giudice measure, which we visualize by the color of every point in the $m_{H}-m_{2 / 3}^{\min }$ plane, where light yellow corresponds to a negligible tuning $\Delta_{\mathrm{BG}} \sim 0$, while dark red depicts a large tuning $\Delta_{\mathrm{BG}} \gtrsim 100$. The measure includes both the tuning to get the correct EWSB (the tuning entering the Higgs vev) as well as a potential ad-hoc tuning in the Higgs mass. In the upper panels of each figure we show the results for the $\mathrm{MCHM}_{5}$ employing $Y_{*}^{q}=0.7$ and $Y_{*}^{q}=1.4$, while in the lower panels we provide the same plots for the $\mathrm{MCHM}_{10}$. As a general trend, one can clearly see that lighter Higgs masses (i.e., lighter partner masses) increase the tuning. This can easily be understood for example from (2.23) and the tuning condition (2.21), (2.22), which leads to an increased tuning for smaller $\beta$. In the left panel of figure $3 \mathrm{a}$, we inspect that for the correct Higgs mass, depicted again by the yellow band, the fine-tuning in the $\mathrm{MCHM}_{5}$ is of the order of $\Delta_{\mathrm{BG}} \sim(10-80)$ for $Y_{*}^{q}=0.7$. For a larger $Y_{*}^{q}=1.4$ the tuning is generically increased since, keeping the dependence on the brane masses, it is determined by the combination $Y_{*}^{q} y^{t}$, which still grows $\sim \sqrt{Y_{*}^{q}}$ for constant $m_{t}$, see (2.12) (and table 1). For that case we see that $\Delta_{\mathrm{BG}} \sim(30-150)$. The qualitative behavior in the $\mathrm{MCHM}_{10}$ is similar, however as the physical $m_{H}$ can hardly be reached, it is difficult to draw quantitative conclusions 

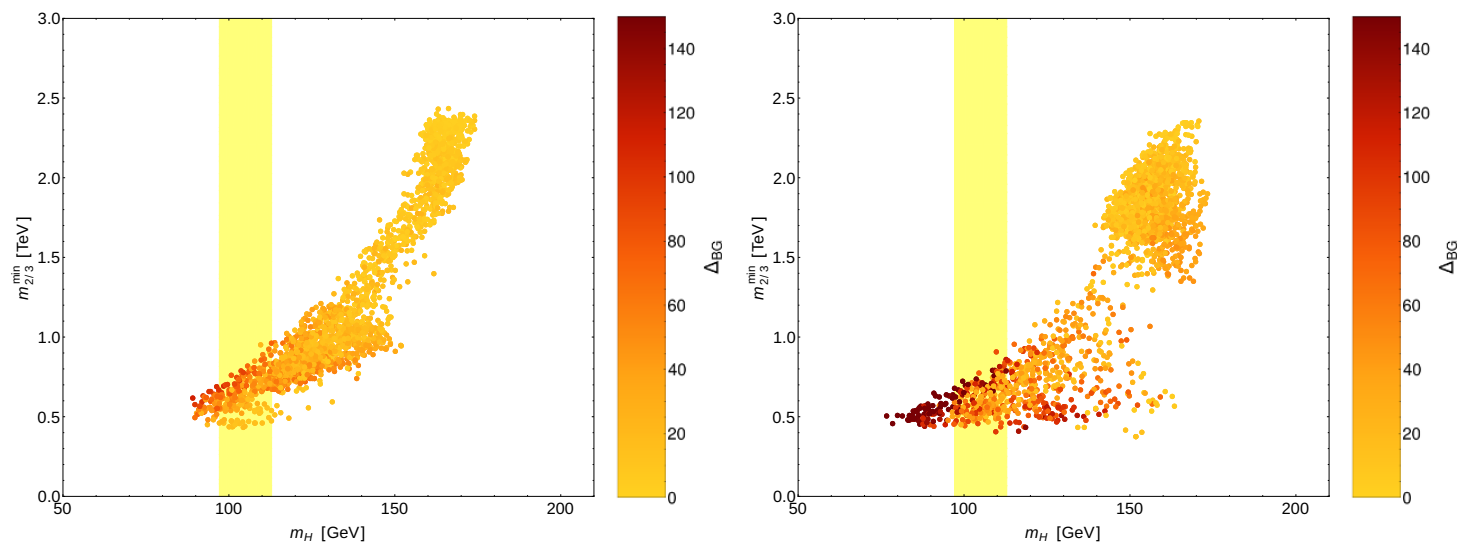

(a) $Y_{*}^{q}=0.7$ (left plot) and $Y_{*}^{q}=1.4$ (right plot) in the $\mathrm{MCHM}_{5}$ with $f_{\pi}=0.8 \mathrm{TeV}$ and $\kappa=\kappa^{\prime}=0$.
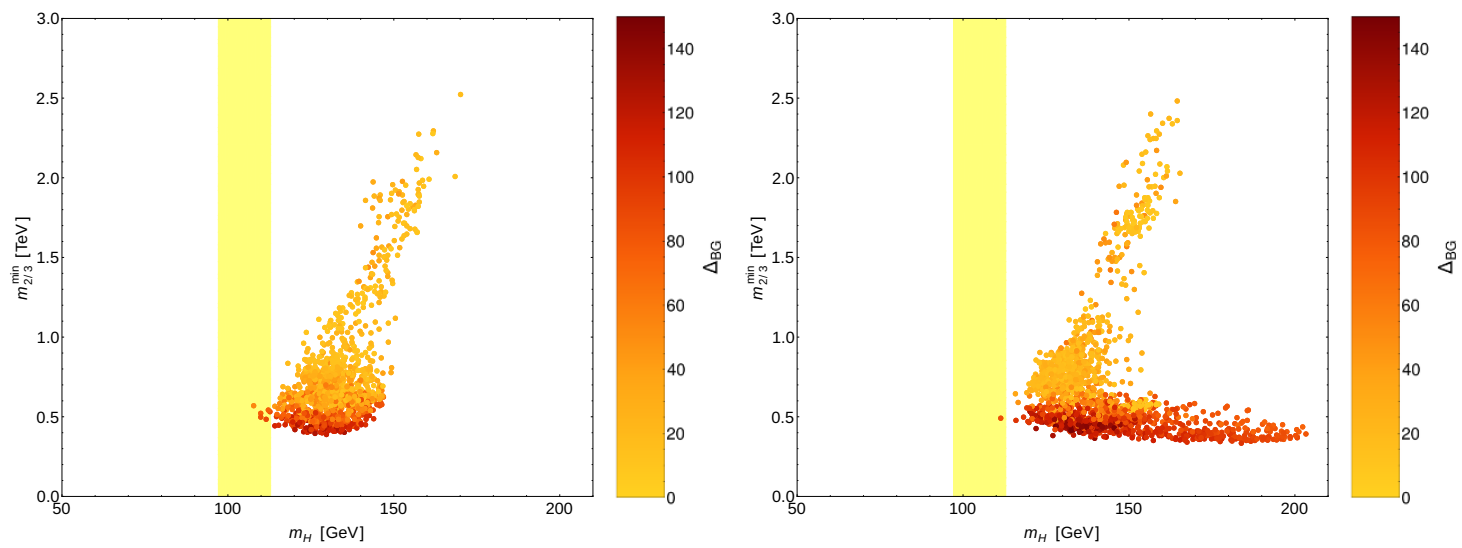

(b) $Y_{*}^{q}=0.7$ (left plot) and $Y_{*}^{q}=1.4$ (right plot) in the $\mathrm{MCHM}_{10}$ with $f_{\pi}=0.8 \mathrm{TeV}$ and $\kappa=\kappa^{\prime}=0$.

Figure 3. Mass of the first top partner as a function of the Higgs mass in the $\mathrm{MCHM}_{5}$ and the $\mathrm{MCHM}_{10}$, figures 3a and 3b, respectively, for $\kappa=\kappa^{\prime}=0$ and $f_{\pi}=0.8 \mathrm{TeV}$. Lighter points correspond to smaller values of $\Delta_{\mathrm{BG}}$ and therefore to less tuned points.

on the tuning. ${ }^{31}$ Increasing the Higgs-decay constant to $f_{\pi}=1 \mathrm{TeV}$ should allow the top partners to be slightly heavier for fixed $m_{H}$, as can be seen from (2.25) (and similarly for the $\mathrm{MCHM}_{10}$ ). This behavior is confirmed in our numerical analysis, presented in figure 4, which shows the same plots as the previous figure, now employing the larger Higgs decay constant. This comes however at the price of a considerably larger tuning, due to a smaller ratio $v / f_{\pi}$, as is obvious from (2.22). In particular for those points that feature the maximal $m_{2 / 3}^{\min }$ of $\sim(900-1000) \mathrm{GeV}$ the tuning becomes in general at least $\Delta_{\mathrm{BG}} \sim \mathcal{O}(100)$.

\footnotetext{
${ }^{31}$ In this particular realization, the $b_{L}$ contribution to the potential becomes relevant, as explained before. As it turns out, especially for the case of large maximal brane masses, this enlarges the parameter region, allowing e.g. $y_{L}^{q}\left(y_{R}^{t}\right)$ to be significantly reduced while the bottom contribution keeps $m_{H}$ fixed as well as potentially saves the EWSB condition (2.21). This new region is characterized by light partner masses, needed (together with sizable brane masses) to arrive at the large $m_{t}$ in the presence of a moderate linear mixing, as well as a large tuning, since the required cancellations in the fermion sector to arrive at a viable EWSB, are now even more challenging.
} 

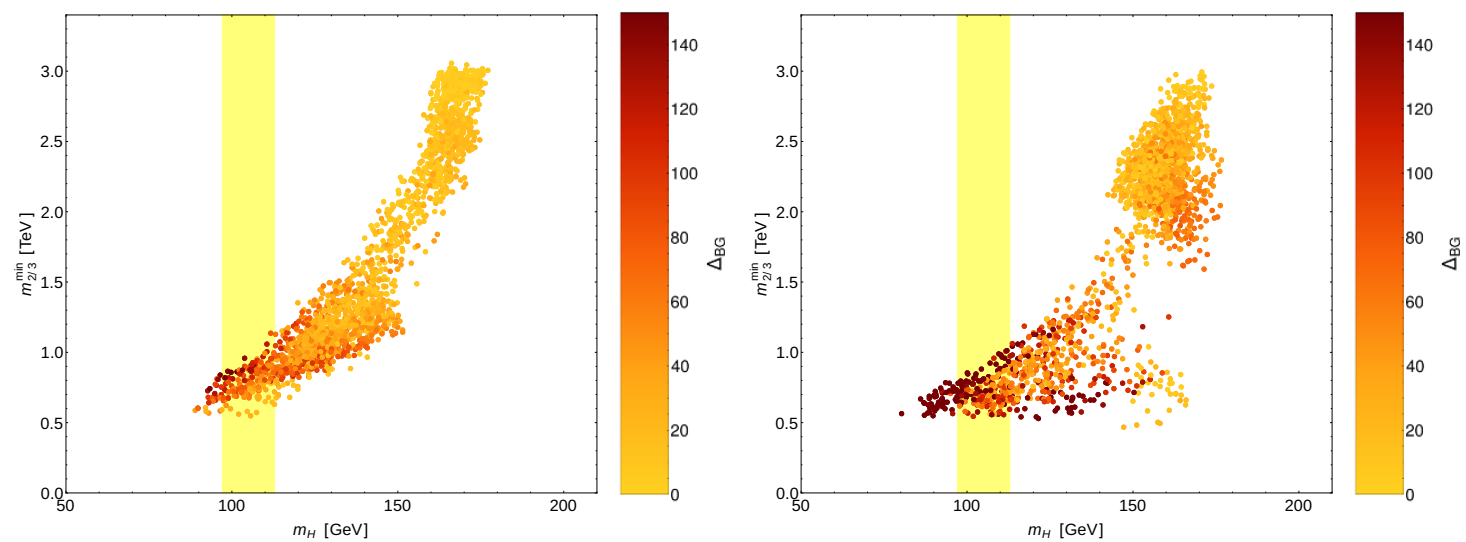

(a) $Y_{*}^{q}=0.7$ (left plot) and $Y_{*}^{q}=1.4$ (right plot) in the $\mathrm{MCHM}_{5}$ with $f_{\pi}=1 \mathrm{TeV}$ and $\kappa=\kappa^{\prime}=0$.
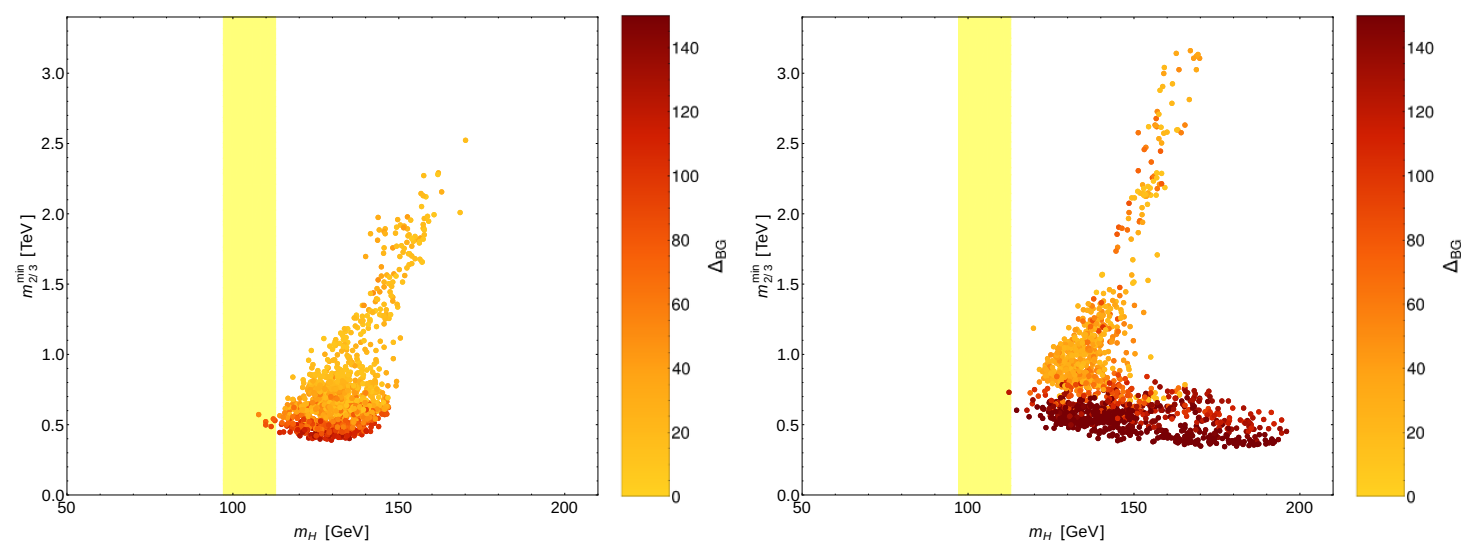

(b) $Y_{*}^{q}=0.7$ (left plot) and $Y_{*}^{q}=1.4$ (right plot) in the $\mathrm{MCHM}_{10}$ with $f_{\pi}=1 \mathrm{TeV}$ and $\kappa=\kappa^{\prime}=0$.

Figure 4. Mass of the first top partner as a function of the Higgs mass in the $\mathrm{MCHM}_{5}$ and the $\mathrm{MCHM}_{10}$, figures $4 \mathrm{a}$ and $4 \mathrm{~b}$, respectively, for $\kappa=\kappa^{\prime}=0$ and $f_{\pi}=1 \mathrm{TeV}$. Lighter points correspond to smaller values of $\Delta_{\mathrm{BG}}$ and therefore to less tuned points.
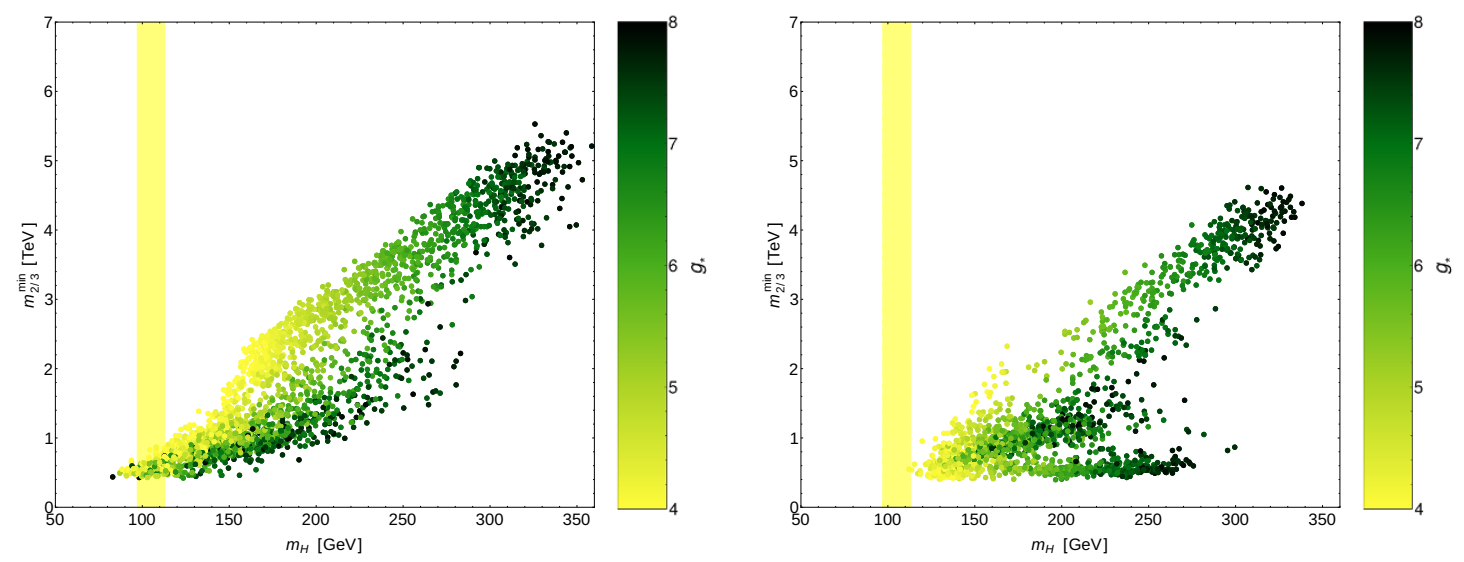

Figure 5. Mass of the first top partner as a function of the Higgs mass in the $\mathrm{MCHM}_{5}$ (left plot) and $\mathrm{MCHM}_{10}$ (right plot). In both cases we have taken $\kappa, \kappa^{\prime} \in[0, \sqrt{3}]$, which corresponds roughly to $g_{*} \in[4,8]$, and have chosen the benchmark value $Y_{*}^{q}=0.7$ as well as $f_{\pi}=0.8 \mathrm{TeV}$. 
Finally, we study in addition the dependence on the 5D gauge coupling $g_{*}$, the "inverse" of the number of colors in the strong sector $N_{\mathrm{CFT}}$ and determined by the coefficient $\kappa$ of the brane localized kinetic terms of the $\mathrm{SU}(2)_{L}$ gauge bosons, see (3.6) and (3.8). In figure 5 we show again the mass of the lightest top partner in dependence on the Higgs mass, for $f_{\pi}=0.8 \mathrm{TeV}$ and $Y_{*}^{q}=0.7$, indicating $g_{*}$ by the hue of the green color. We see that if the $5 \mathrm{D}$ gauge coupling is increased with respect to the default value $g_{*} \sim 4$ (by turning on the brane kinetics), which corresponds to a darker green, the Higgs boson gets generally heavier for a given $m_{2 / 3}^{\min }$. This can be understood easily as the increased 5D coupling enhances all contributions to the potential. While in the $\mathrm{MCHM}_{5}$, shown in the left plot, it is still possible to arrive at the correct Higgs mass, in the $\mathrm{MCHM}_{10}$ with non-negligible brane kinetics, the Higgs becomes unavoidably much too heavy. The fact that the Higgs mass generically increases for a given mass of the lightest top partner directly means that also changing $N_{\mathrm{CFT}}$ can not raise the mass of the light partners, induced by $m_{H}$.

In summary, our numerical analysis confirms the qualitative features described in section 2.2. Models mixing the different SM chiralities with a fundamental or an adjoint representation require light partners below the $\mathrm{TeV}$ scale. Moreover, they feature a larger tuning than the naive estimate of $v^{2} / f_{\pi}^{2} \sim 10 \%$. We will now see quantitatively how a composite lepton sector can naturally break the correlation between $m_{2 / 3}^{\min }$ and $m_{H}$, without the need to change the colored particle content.

\subsubsection{The impact of leptons}

We start with the standard models of $\tau_{R}$ compositeness due to flavor protection, without a unification of right-handed lepton and neutrino multiplets, i.e., the ordinary $\mathrm{MCHM}_{5}^{5-14}$. In figure $6 \mathrm{a}$, we show again the mass of the lightest $Q=2 / 3$ resonance, as a function of the Higgs mass for $f_{\pi}=0.8 \mathrm{TeV}$, assuming once more $Y_{*}^{q}=0.7$ and $Y_{*}^{q}=1.4$ for the maximally allowed brane masses in the quark sector. For the leptons, we employ $Y_{*}^{l}=0.35$, accounting for the 'Yukawa suppression' generally present in these models, and here and in the following again assume no brane localized kinetic terms. The plots confirm nicely our discussion of section 2.3. The basically unsuppressed lepton contributions to $\beta$, that arise for a non-negligible mixing with a $\mathbf{1 4}$, allow for a destructive interference with the quark contributions such as to raise the masses of the lightest top partners well beyond the $1 \mathrm{TeV}$ scale. In particular, for both values of the brane masses considered, lightest partners as heavy as $2 \mathrm{TeV}$ are possible, removing considerably the pressure coming from current LHC searches. Due to the same mechanism, the Higgs mass could now be even below $100 \mathrm{GeV}$ and its measured value lies not anymore at the boundary of the possible values. Because of the modest leptonic contribution to $\beta$, the models also feature no ad-hoc tuning. As before, the general tuning $\Delta_{\mathrm{BG}}$ is visualized by the hue of the points in the $m_{H}-m_{2 / 3}^{\min }$ plane. One can see that the lepton sector adds no additional relevant tuning to the setup and the dependence on $m_{H}$ follows the same pattern as in the $\mathrm{MCHM}_{5}$, dominated by the large top contribution to $\alpha$ which needs to be tuned to become of the order of $\beta$. Also the increased tuning for larger $Y_{*}^{q}$ can be explained along the same lines as before. An interesting feature however becomes apparent, if one studies the tuning versus $m_{2 / 3}^{\min }$. The tuning is decreased for heavier top partners. So, not only are light partners not necessary anymore in the setup, 

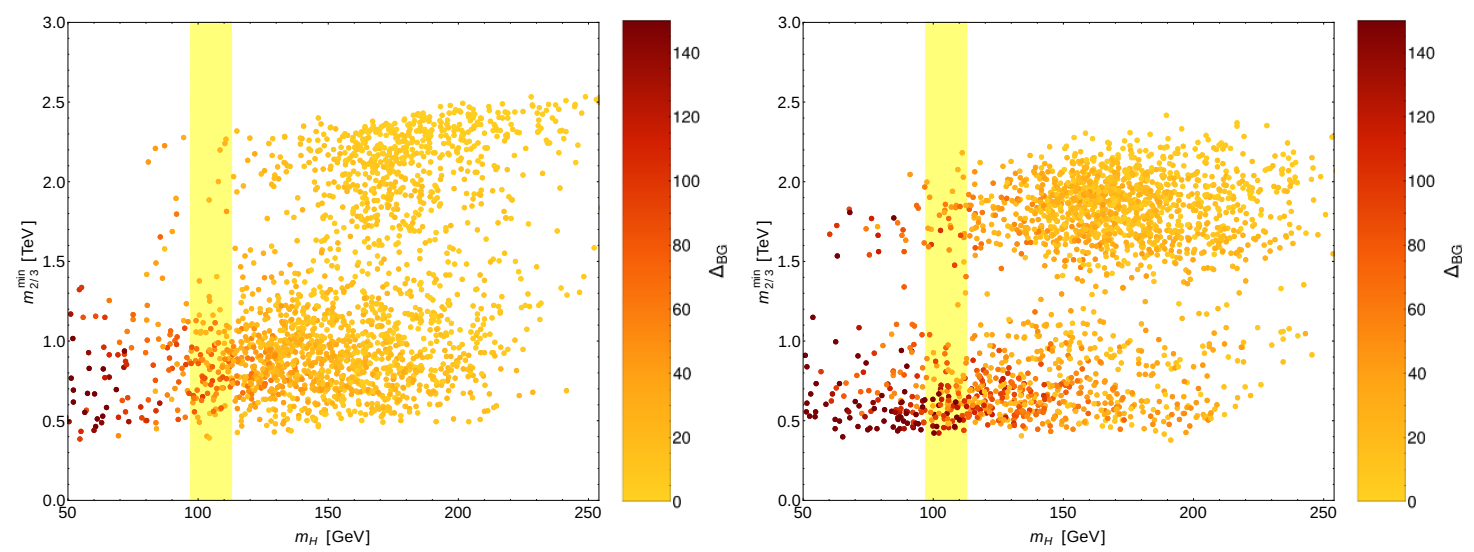

(a) $Y_{*}^{q}=0.7$ (left plot) and $Y_{*}^{q}=1.4$ (right plot) in the $\mathrm{MCHM}_{5}^{5-14}$ with $f_{\pi}=0.8 \mathrm{TeV}$ and $\kappa=\kappa^{\prime}=0$.
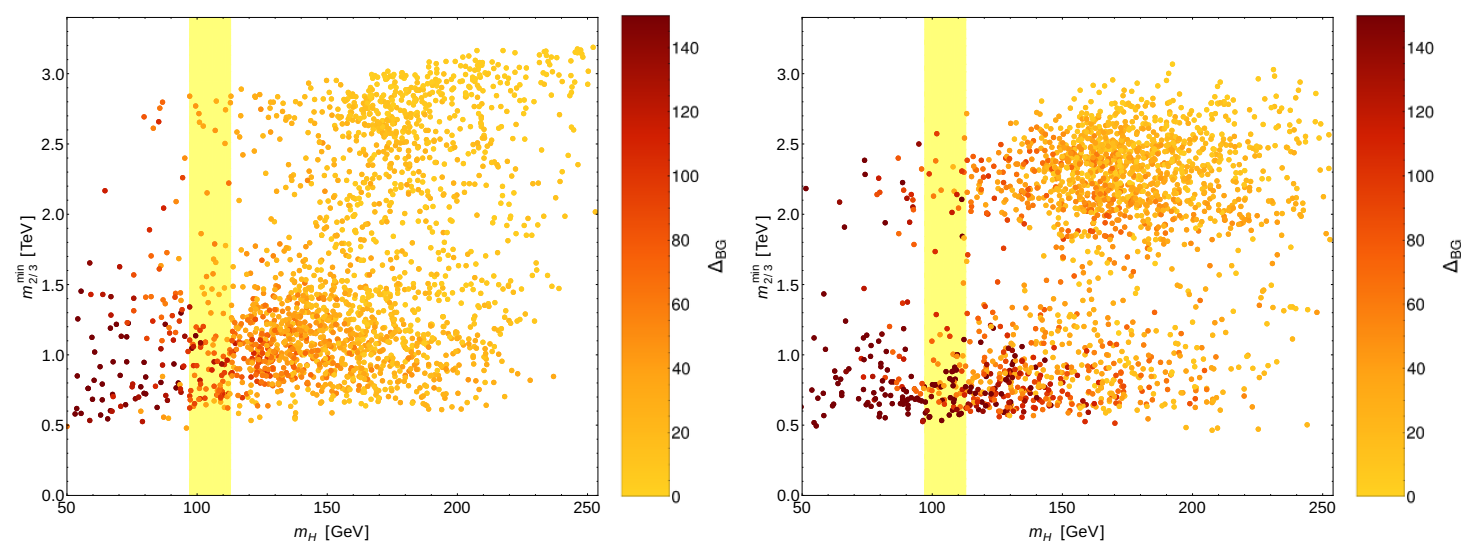

(b) $Y_{*}^{q}=0.7$ (left plot) and $Y_{*}^{q}=1.4$ (right plot) in the $\mathrm{MCHM}_{5}^{5-14}$ with $f_{\pi}=1 \mathrm{TeV}$ and $\kappa=\kappa^{\prime}=0$.

Figure 6. Mass of the first top partner as a function of the Higgs mass in the $\mathrm{MCHM}_{5}^{5-14}$ for $f_{\pi}=0.8 \mathrm{TeV}$ and $f_{\pi}=1 \mathrm{TeV}$, figures $6 \mathrm{a}$ and $6 \mathrm{~b}$, respectively. In both cases we have fixed $\kappa=\kappa^{\prime}=0$, while $Y_{*}^{l}=0.35$. Lighter points correspond to smaller values of $\Delta_{\mathrm{BG}}$ and therefore to less tuned points.

they are even less natural and the expectation would correspond to top partners $\gtrsim 1.5 \mathrm{TeV}$. This can be understood since points with a larger $m_{2 / 3}^{\min }$ feature usually a larger $y_{t}$ and this makes the "double tuning" (2.22) less severe. All these qualitative features remain after increasing the Higgs decay constant to $f_{\pi}=1 \mathrm{TeV}$, which is shown in $6 \mathrm{~b}$. The slightly higher partner masses and the increase in the tuning can be explained as before in the $\mathrm{MCHM}_{5,10}$.

To show that our solution does not introduce ultra-light $\tau$ partners in conflict with observation, we show in figure 7 the same plots as before (with $f_{\pi}=0.8 \mathrm{TeV}$ ), now indicating the mass of the lightest $Q=-1$ resonance, $m_{-1}^{\min }$, by the hue of the blue color. We inspect that the $\tau$ partners are in general heavier than a TeV. In particular, there are always points with both $m_{2 / 3}^{\min }>2 \mathrm{TeV}, m_{-1}^{\min }>2 \mathrm{TeV}$.

We now move on to compare more quantitatively the predictions for $m_{2 / 3}^{\min }$ in the $\mathrm{MCHM}_{5}^{5-14}$ with the minimal quark setups, where we take the $\mathrm{MCHM}_{5}$ as a reference. Checking the corresponding survival function for the $\mathrm{MCHM}_{5}^{5-14}$ (green solid) against the 

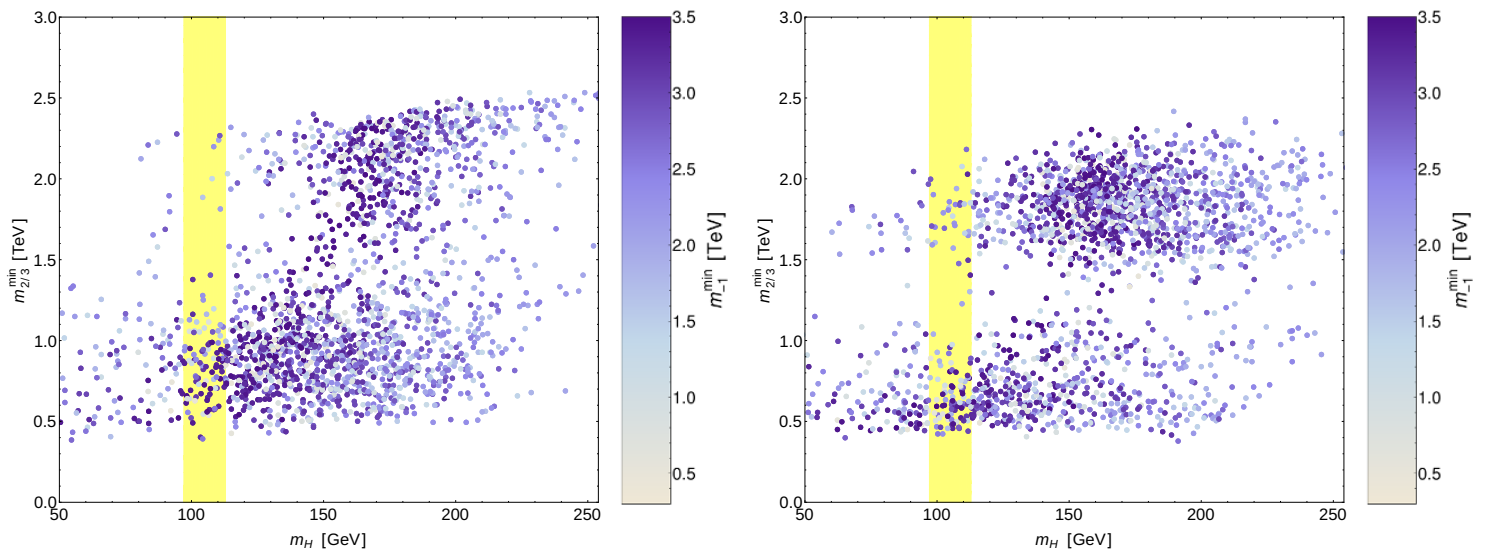

Figure 7. Mass of the first top partner as a function of the Higgs mass in the $\mathrm{MCHM}_{5}^{5-14}$ for $Y_{*}^{q}=0.7$ (left plot) and $Y_{*}^{q}=1.4$ (right plot), where always $f_{\pi}=0.8 \mathrm{TeV}, \kappa=\kappa^{\prime}=0$, and $Y_{*}^{l}=0.35$. Lighter points correspond to smaller values of $m_{-1}^{\min }$.
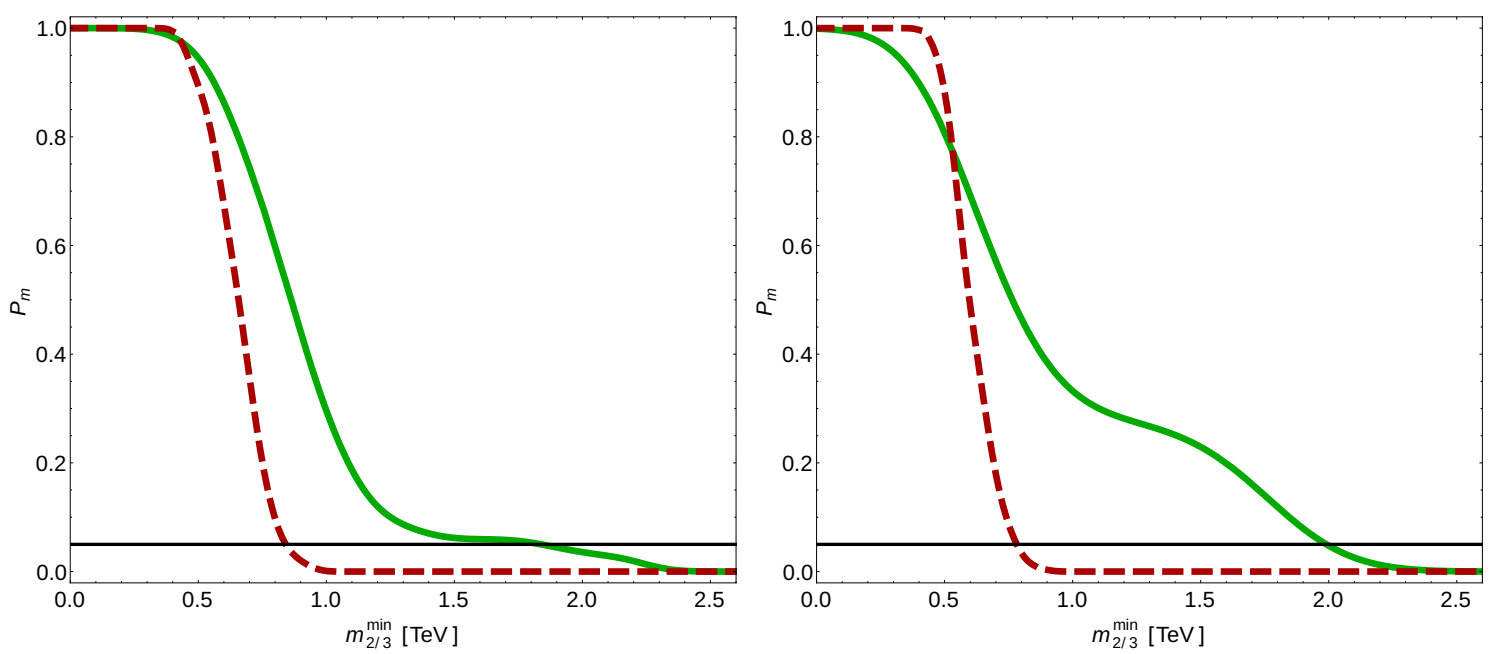

Figure 8. Survival function $\mathcal{P}_{m}$ of the first top partner mass for $Y_{*}^{q}=0.7$ (left plot) and $Y_{*}^{q}=1.4$ (right plot). In both cases we have plotted the $\mathrm{MCHM}_{5}^{5-14}$ (solid green) with $Y_{*}^{l}=0.35$ vs. the $\mathrm{MCHM}_{5}$ (dashed red) for $\kappa=\kappa^{\prime}=0$ and $f_{\pi}=0.8 \mathrm{TeV}$, assuming $m_{H}\left(f_{\pi}\right)=105 \mathrm{GeV}(1 \pm 7.5 \%)$.

one of the $\mathrm{MCHM}_{5}$ (red-dashed) in the left panel of figure 8 , where $Y_{*}^{q}=0.7$ and always $f_{\pi}=0.8 \mathrm{TeV}$, one can see that while $95 \%(99 \%)$ of the parameter points of the $\mathrm{MCHM}_{5}$ feature light partners below $800 \mathrm{GeV}(950 \mathrm{GeV})$, in the $\mathrm{MCHM}_{5}^{5-14}$ still $60 \%$ (35\%) of the points have $m_{2 / 3}^{\min }$ bigger than these values. In particular the $95 \%$ quantile is reached only for $m_{2 / 3}^{\min }=1.8 \mathrm{TeV}$. The difference becomes in general even more dramatic for larger brane masses, $Y_{*}^{q}=1.4$, shown in the right panel of figure 8. While the values for the $\mathrm{MCHM}_{5}$ are very similar, for the $\mathrm{MCHM}_{5}^{5-14}$ now even $25 \%$ of the points feature $m_{2 / 3}^{\min }>1.5 \mathrm{TeV}$. This can be understood from the fact that in that case the large top mass allows more easily larger values of the partner masses, see (2.12), while the correct Higgs mass can still be obtained via the lepton contributions. 

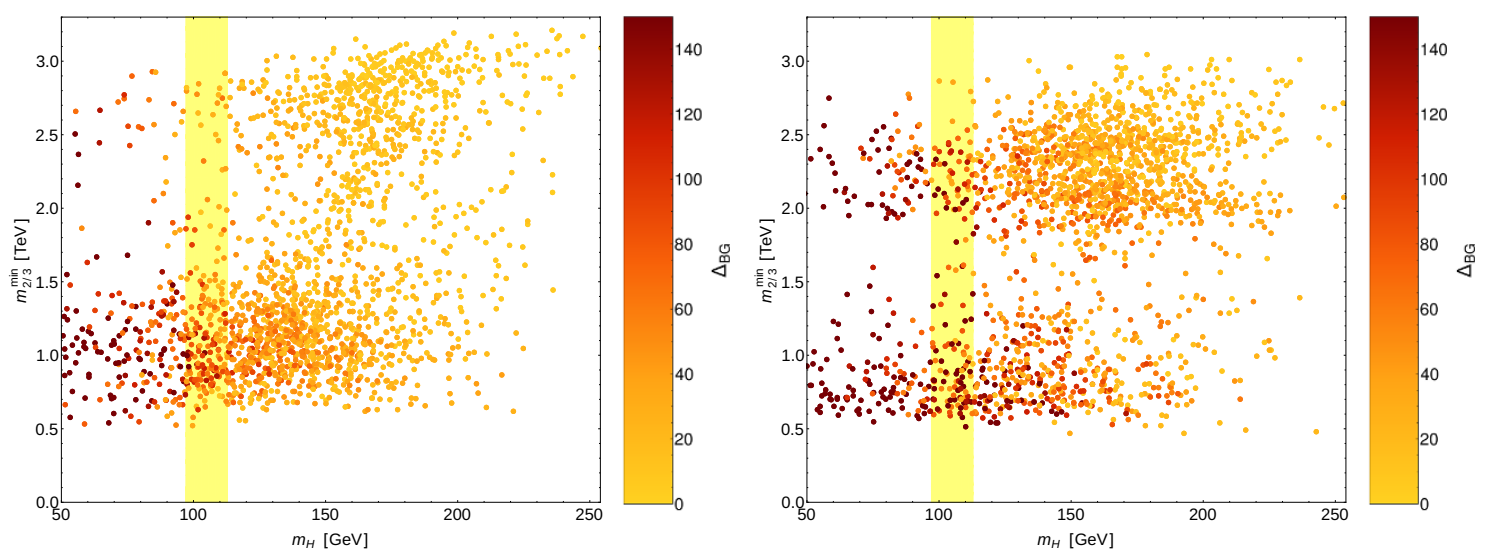

Figure 9. Mass of the first top partner as a function of the Higgs mass in the $\mathrm{MCHM}_{5}^{14}$ for $Y_{*}^{q}=0.7$ (left plot) and $Y_{*}^{q}=1.4$ (right plot), where always $Y_{*}^{l}=0.35, f_{\pi}=1 \mathrm{TeV}$, and $\kappa=\kappa^{\prime}=0$. Lighter points correspond to smaller values of $\Delta_{\mathrm{BG}}$ and therefore to less tuned points.
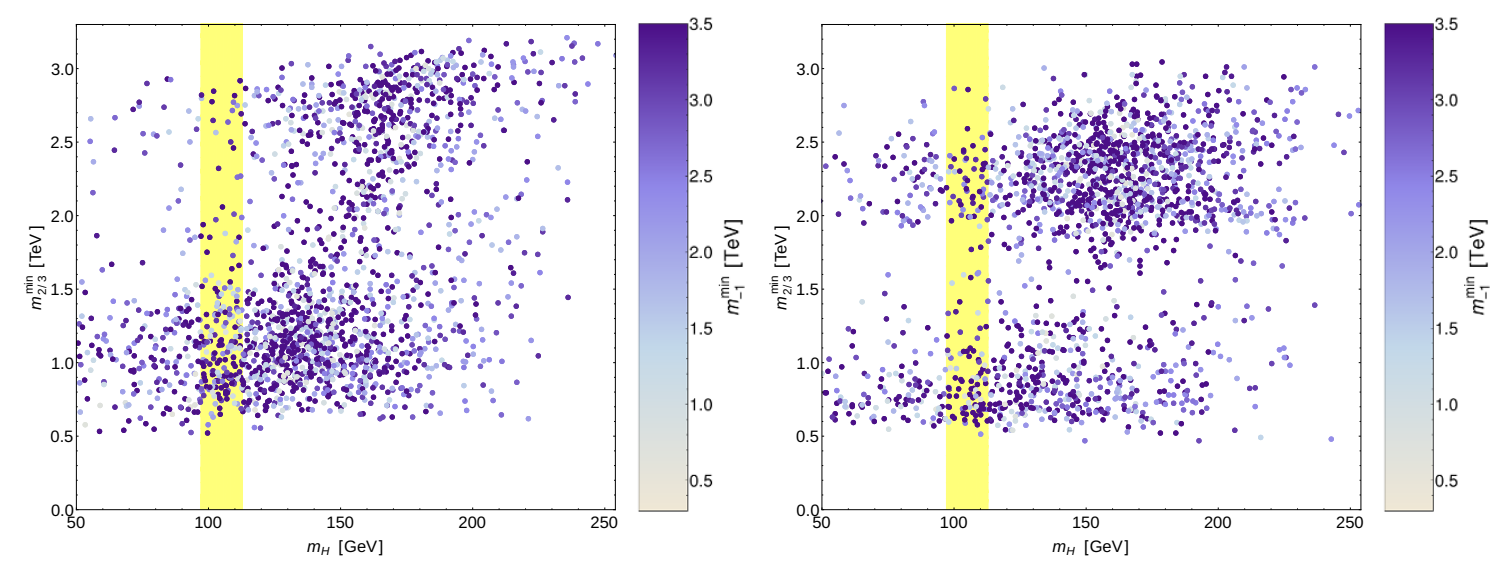

Figure 10. Mass of the first top partner as a function of the Higgs mass in the $\mathrm{MCHM}_{5}^{14}$ for $Y_{*}^{q}=0.7$ (left plot) and $Y_{*}^{q}=1.4$ (right plot), where always $Y_{*}^{l}=0.35, f_{\pi}=1 \mathrm{TeV}$, and $\kappa=\kappa^{\prime}=0$. Lighter points correspond to smaller values of $m_{-1}^{\min }$.

For completeness we also present the corresponding predictions for the $\mathrm{MCHM}_{5}^{14}$, where both the left-handed and right-handed leptons are embedded in symmetric representations of $\mathrm{SO}(5)$, in figures 9-11. Note that here we show only the results for $f_{\pi}=1 \mathrm{TeV}$, since the model generically features larger corrections to the Higgs couplings, compared to the $\mathrm{MCHM}_{5}^{5-14}$-like setup, which has a more efficient protection, as can be seen from the results of section 3.1.3 and taking into account that the infinite KK sum is saturated to a large extend by the first mode. As expected, although some numerical details change, the qualitative behavior is not modified by mixing the (elementary) left handed leptons with another representation. The fine tuning, depicted in figure 9 , is similar to the case of $f_{\pi}=1 \mathrm{TeV}$ examined before, and the same is true for the masses of the $\tau$ partners, which are still not required to be light, see figure 10. In the end, in the $\mathrm{MCHM}_{5}^{14}$ with $f_{\pi}=1 \mathrm{TeV}$ the top partners can be lifted to high values of $m_{2 / 3}^{\min } \gtrsim 2.5 \mathrm{TeV}$ without going to extreme corners of the parameter space, see figure 11. 

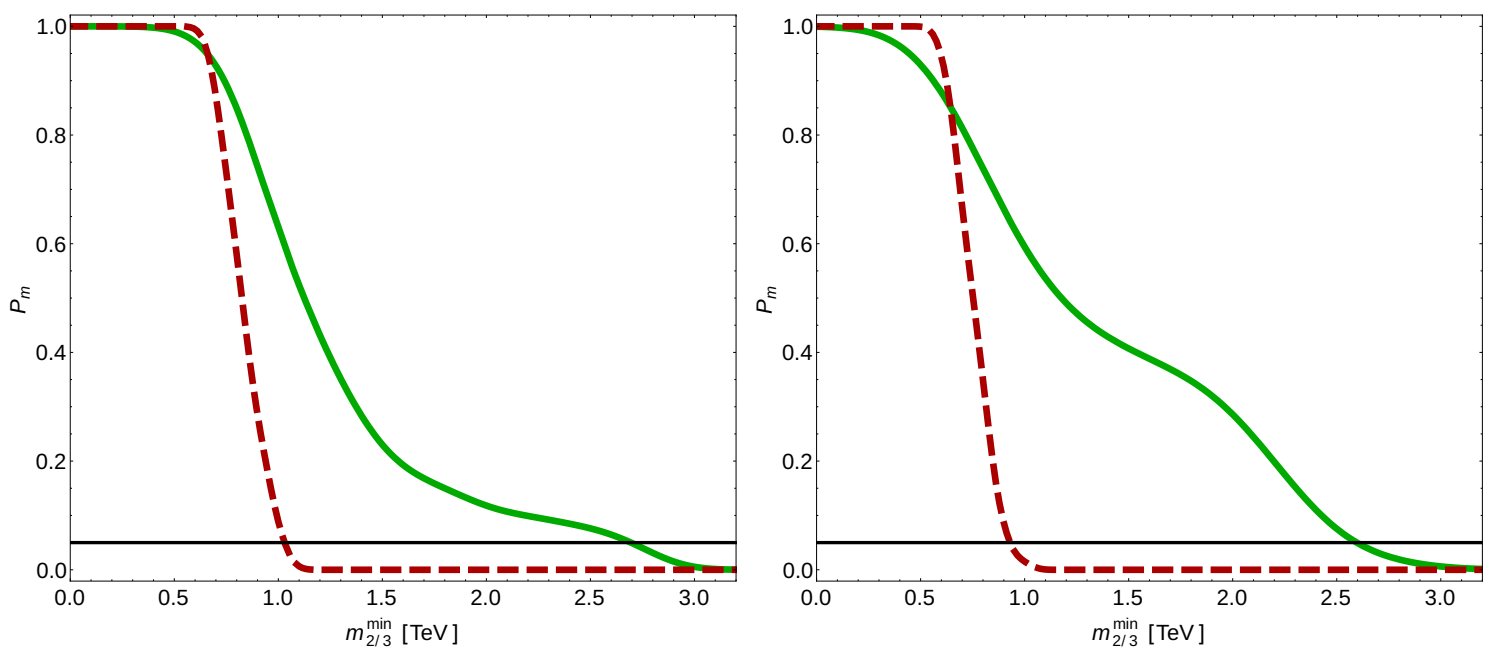

Figure 11. Survival function $\mathcal{P}_{m}$ of the first top partner mass for $Y_{*}^{q}=0.7$ (left plot) and $Y_{*}^{q}=1.4$ (right plot). In both cases we have plotted the $\mathrm{MCHM}_{5}^{14}$ (solid green) with $Y_{*}^{l}=0.35$ vs. the $\mathrm{MCHM}_{5}$ (dashed red), for $\kappa=\kappa^{\prime}=0$ and $f_{\pi}=1 \mathrm{TeV}$, assuming $m_{H}\left(f_{\pi}\right)=105 \mathrm{GeV}(1 \pm 7.5 \%)$.

Let us continue with more minimal models of the lepton sector. We start with the minimal type-III seesaw model $\mathrm{mMCHM}_{5}^{\mathrm{III}}$, introduced before - assuming still a Yukawa suppression due to flavor symmetries or the like, i.e., $Y_{*}^{l}=0.35$. We study again the mass of the lightest $Q=2 / 3$ resonance, as a function of the Higgs mass, which we display in figure 12 , assuming once more $Y_{*}^{q}=0.7$ and $Y_{*}^{q}=1.4$, as well as $f_{\pi}=0.8 \mathrm{TeV}$. As before, the tuning $\Delta_{\mathrm{BG}}$ is visualized by the hue of the points in the $m_{H}-m_{2 / 3}^{\min }$ plane. It is evident from the plots that this model, as expected, still allows to lift the masses of the lightest top partner well beyond a $\mathrm{TeV}$, without increasing the tuning. On the contrary, just as for the $\mathrm{MCHM}_{5}^{5-14}$, heavier top partners are more natural in this model (which is not true for models with negligible impact of the lepton sector). Compared to the $\mathrm{MCHM}_{5}^{5-14}$, due to the $N_{g}$-enhanced leptonic contribution in the $\mathrm{mMCHM}_{5}^{\mathrm{III}}$, the fraction of the parameter space featuring $m_{2 / 3}^{\min } \sim(1-2.5) \mathrm{TeV}$ for a viable $m_{H}$ (displayed again by the yellow band) is however significantly enhanced. Since a considerable portion of these points features a tuning of $\Delta_{\mathrm{BG}} \lesssim(10-20)$ the model even allows to reconcile the absence of light partners with the minimization of the fine tuning. ${ }^{32}$ Moreover, the masses of the lepton partners are in general at the high scale $m_{\Psi}$ in the type-III seesaw models, as the IR localization of the right-handed leptons is rather modest (and $m_{\ell} \ll m_{t}$ ).

For completeness we also examine the $\mathrm{mMCHM}_{5}^{\mathrm{III}>}$, i.e., we lift the 'Yukawa suppression' so that we now study, in figure 13, the cases of $Y_{*}^{l}=Y_{*}^{q}=0.7$ (left plot) and $Y_{*}^{l}=Y_{*}^{q}=1.4$ (right plot), while the other parameters remain as given before. While the qualitative situation remains similar, now the more pronounced leptonic contribution leads to a further increase of the average $m_{2 / 3}^{\min }$ in the viable $m_{H}$ region, which however now features slightly less points. The increase is due to the fact that in this class of models

\footnotetext{
${ }^{32}$ Still the model features no "ad-hoc" tuning and the observed $m_{H}$ remains in a rather central region of the parameter space.
} 

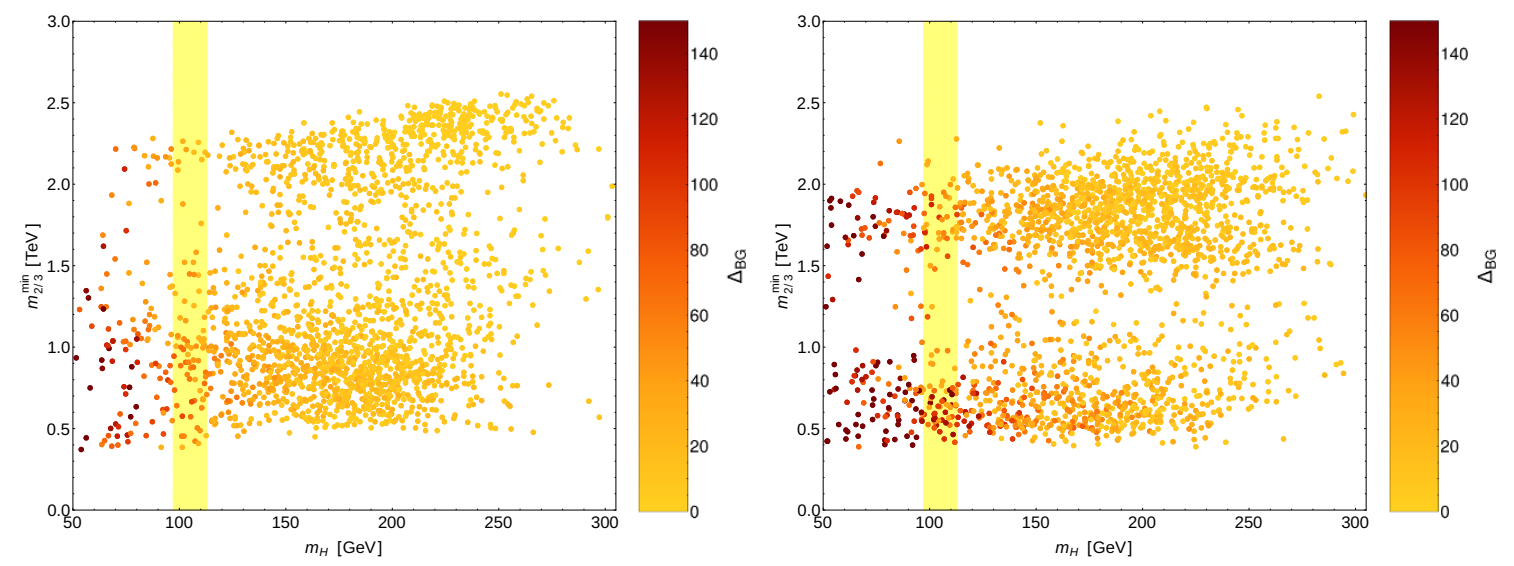

Figure 12. Mass of the first top partner as a function of the Higgs mass in the $\mathrm{mMCHM}_{5}^{\mathrm{III}}$ for $Y_{*}^{q}=0.7$ (left plot) and $Y_{*}^{q}=1.4$ (right plot). In both cases we have fixed $Y_{*}^{l}=0.35, \kappa=\kappa^{\prime}=0$, as well as $f_{\pi}=0.8 \mathrm{TeV}$. Lighter points correspond to smaller values of $\Delta_{\mathrm{BG}}$ and therefore to less tuned points.
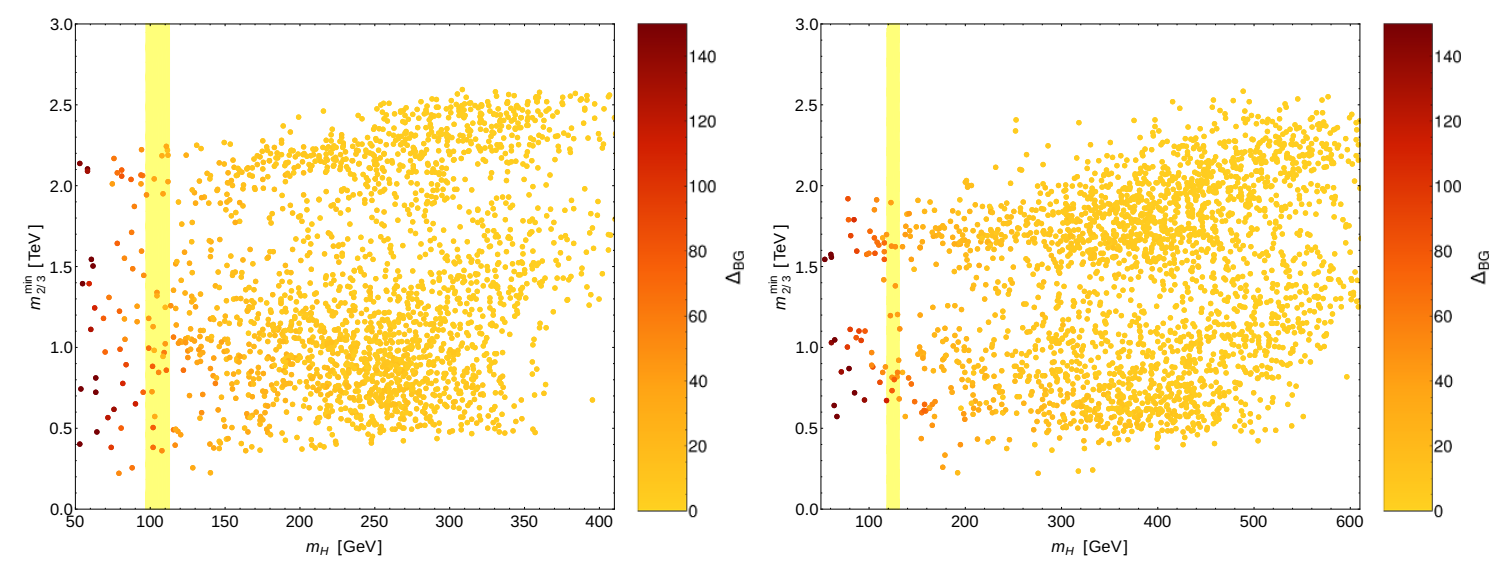

Figure 13. Mass of the first top partner as a function of the Higgs mass in the $\mathrm{mMCHM}_{5}^{\mathrm{III}>}$ for $Y_{*}^{l}=Y_{*}^{q}=0.7$ (left plot) and $Y_{*}^{l}=Y_{*}^{q}=1.4$ (right plot). In both cases we have fixed $\kappa=\kappa^{\prime}=0$ and $f_{\pi}=0.8 \mathrm{TeV}$. Lighter points correspond to smaller values of $\Delta_{\mathrm{BG}}$ and therefore to less tuned points.

the localization of the right handed leptons is mostly fixed by the Majorana masses and remains constant under changes of $Y_{*}^{l}$.

The distribution of the partner masses in the minimal lepton models is examined in more detail in figure 14 where we display the survival function for the first top partner mass in the $\mathrm{mMCHM}_{5}^{\mathrm{III}}$ (green solid, left panel) as well as in the $\mathrm{mMCHM}_{5}^{\mathrm{III}>}$ (green solid, right panel), employing $Y_{*}^{l}=0.35$ and $Y_{*}^{l}=0.7$, respectively, always against the one in the $\mathrm{MCHM}_{5}$ (red-dashed), with $Y_{*}^{q}=0.7$, and always $f_{\pi}=0.8 \mathrm{TeV}$. Although the size of the brane masses is still rather modest, we can see a large relaxation of the need for light partners. For the case of the $\mathrm{mMCHM}_{5}^{\mathrm{III}}$, still $\gtrsim 10 \%$ of the parameter space (in 

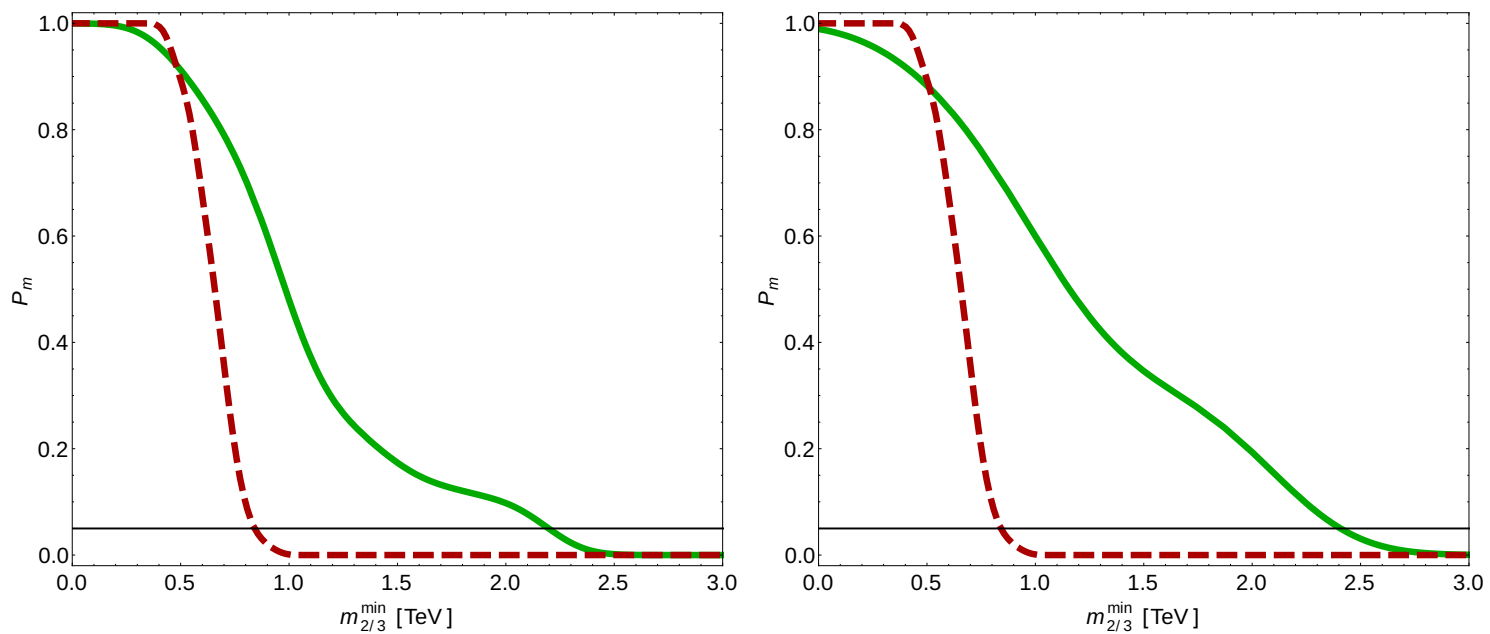

Figure 14. Survival function $\mathcal{P}_{m}$ of the first top partner mass in the $\mathrm{mMCHM}_{5}^{\mathrm{III}}$, with $Y_{*}^{l}=0.35$ (solid green, left plot) and in the $\mathrm{mMCHM}_{5}^{\mathrm{III}>}$, with $Y_{*}^{l}=0.7$ (solid green, right plot), plotted in each case vs. the $\mathrm{MCHM}_{5}$ (dashed red). We always assume $Y_{*}^{q}=0.7, \kappa=\kappa^{\prime}=0, f_{\pi}=0.8 \mathrm{TeV}$, as well as $m_{H}\left(f_{\pi}\right)=105 \mathrm{GeV}(1 \pm 7.5 \%)$.

agreement with the Higgs mass) features partner masses of even $m_{2 / 3}^{\min } \gtrsim 2 \mathrm{TeV}$ while for the $\mathrm{mMCHM}_{5}^{\mathrm{III}>}$ the same holds true for $\sim 20 \%$ of the viable points.

Finally, we explore the most minimal of all models of the composite framework studied in this work, the $\mathrm{mMCHM}_{5-1}^{\mathrm{III}}$. With this model, we expect to leave finally the class of setups getting along without any ad-hoc tuning. The contribution $\alpha_{l}$ due to the leptons in the symmetric representation of $\mathrm{SO}(5)$ is now required to cancel to a significant extend the sizable contribution of the top quark to allow for EWSB and, as discussed in section 2, the now also large $\beta_{l}$ will generically lead to a relatively large $m_{H} \cdot{ }^{33}$ Indeed, we inspect in figure 15 , which shows again $\Delta_{\mathrm{BG}}$ in the $m_{H}-m_{2 / 3}^{\min }$ plane, assuming $f_{\pi}=0.8 \mathrm{TeV}$, that for very light Higgs masses the tuning raises strongly, already for the lower brane masses. However, although the model belongs to the category of ad-hoc tuning, in the end the situation is still not so bad and the correct Higgs mass can be reached with a modest tuning of $\Delta_{\mathrm{BG}} \sim(30-40)$ in the case of $Y_{*}^{q}=Y_{*}^{l}=0.7$ (left panel), while even for $Y_{*}^{q}=Y_{*}^{l}=1.4$ (right panel) the corresponding tuning is rather moderate, about $\Delta_{\mathrm{BG}} \sim(40-80)$. As explained before, the increase is not excessive in particular due to the option of a relatively fully composite $t_{R}$, not contributing to $V(h)$ in this setup, which in turn allows for a less IR localized $t_{L}$ and thus a reduced quark contribution to the Higgs potential. As is clearly visible from the plot, the model does not even feature ultra light partners below a $\mathrm{TeV}$ anywhere in its parameter space - the discovery of such states could exclude the $\mathrm{mMCHM}_{5-1}^{\mathrm{III}>}$. The corresponding survival functions, depicted by

\footnotetext{
${ }^{33}$ While already in the intermediate case of the $\mathrm{mMCHM}_{5}^{\mathrm{III}>}$ the leptonic contribution could be large, the model still allowed for more freedom, compared to the $\mathrm{mMCHM}_{5-1}^{\mathrm{III}>}$, which has less parameters and where a sizable leptonic contribution to $\beta$ is unavoidable due to the large top mass. However, compared to the $\mathrm{mMCHM}_{5}^{\mathrm{III}>}$, the even more natural $\mathrm{mMCHM}_{5}^{\mathrm{III}}$ seems to be the preferred way out of the light partner issue with a light Higgs.
} 

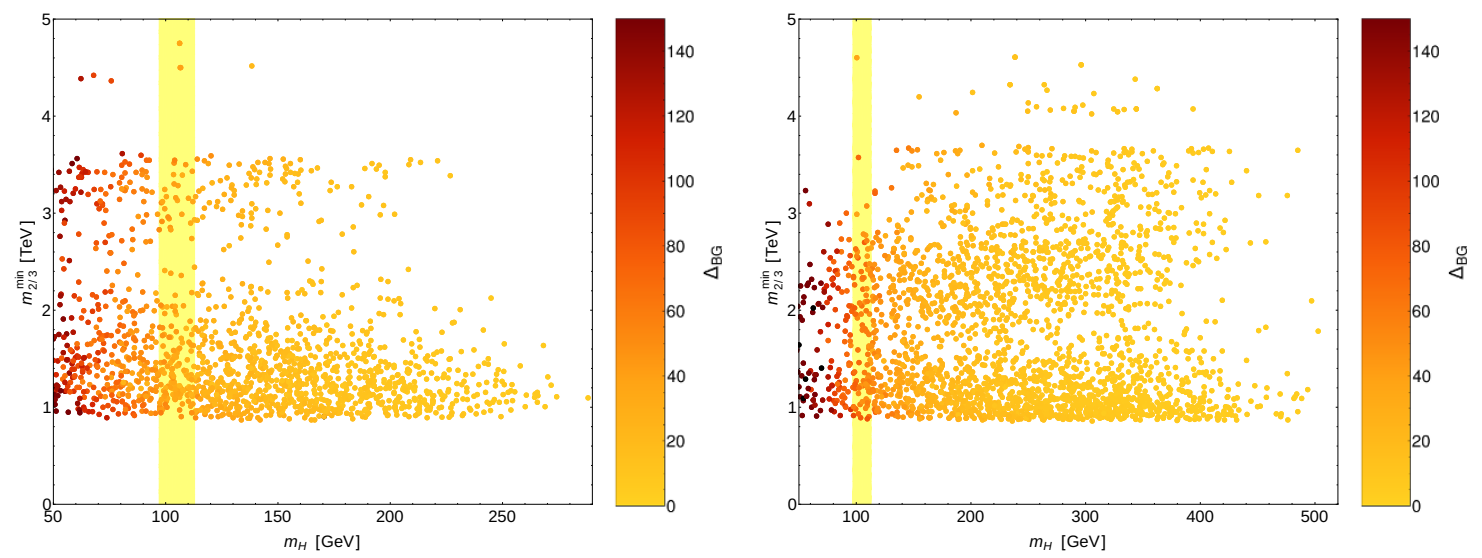

Figure 15. Mass of the first top partner as a function of the Higgs mass in the mMCHM $\mathrm{ML}_{5-1}^{\mathrm{III}>}$ with $Y_{*}^{l}=Y_{*}^{q}=0.7$ (left plot) and $Y_{*}^{l}=Y_{*}^{q}=1.4$ (right plot). In both cases we have again taken $\kappa=\kappa^{\prime}=0$ and $f_{\pi}=0.8 \mathrm{TeV}$. Lighter points correspond to smaller values of $\Delta_{\mathrm{BG}}$ and therefore to less tuned points.
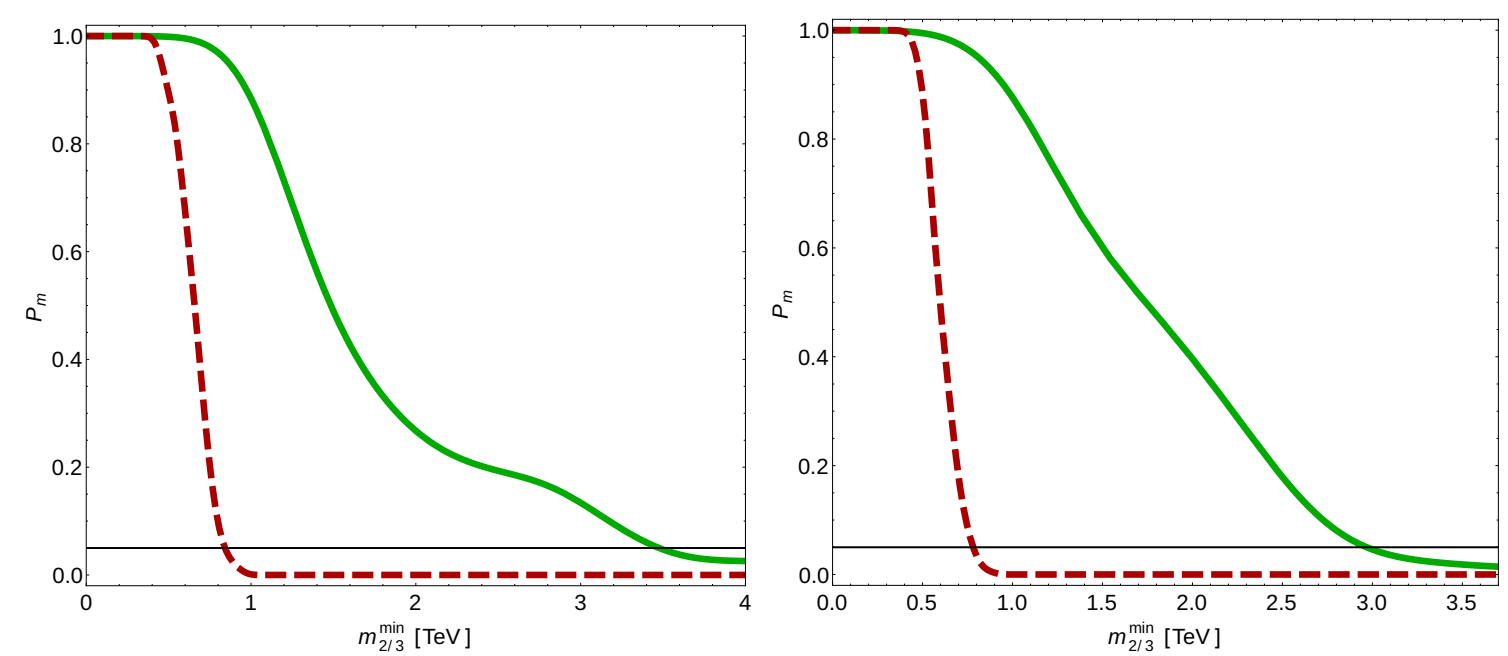

Figure 16. Survival function $\mathcal{P}_{m}$ of the first top partner mass in the $\mathrm{mMCHM}_{5-1}^{\mathrm{III}>}$ with $Y_{*}^{l}=$ $Y_{*}^{q}=0.7$ (solid green, left plot) and $Y_{*}^{l}=Y_{*}^{q}=1.4$ (solid green, right plot), plotted in each case vs. the $\mathrm{MCHM}_{5}$ (dashed red) with $Y_{*}^{q}=0.7$ and $Y_{*}^{q}=1.4$, respectively. In both cases we assume $\kappa=\kappa^{\prime}=0, f_{\pi}=0.8 \mathrm{TeV}$, as well as $m_{H}\left(f_{\pi}\right)=105 \mathrm{GeV}(1 \pm 7.5 \%)$.

the solid green lines in figure 16, do not drop under $5 \%$ even until $m_{2 / 3}^{\min } \gtrsim 3 \mathrm{TeV}$ both for $Y_{*}^{l}=Y_{*}^{q}=0.7$ (left plot) and $Y_{*}^{l}=Y_{*}^{q}=1.4$ (right plot).

After presenting the numerical results for the models that allow to lift the top-partner masses via significant leptonic contributions, we now want to compare them quantitatively to models that employ a $\mathbf{1 4}$ in the quark sector to lift the partner masses. For these models we expect in general a sizable ad-hoc tuning, as explained in section 2 . We start with the $\mathrm{MCHM}_{14}$, where both top chiralities mix with a $\mathbf{1 4}$ of SO(5). Looking at the $m_{H}-m_{2 / 3}^{\min }$ plane presented in figure 17, we can on the one hand see explicitly the option to lift the lightest top partners beyond a $\mathrm{TeV}$ in part of the parameter space. On the other hand, 

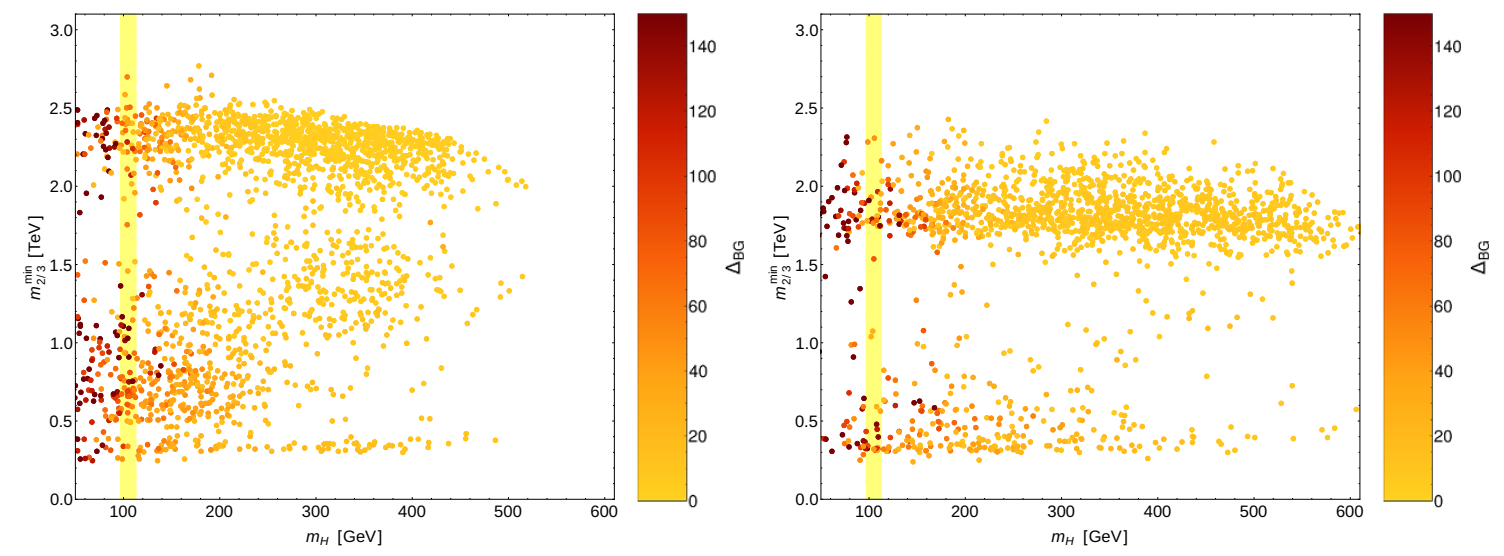

Figure 17. Mass of the first top partner as a function of the Higgs mass in the $\mathrm{MCHM}_{14}$ for $Y_{q}^{*}=0.7$ (left) and $Y_{q}^{*}=1.4$ (right). In both cases we have assumed $f_{\pi}=0.8 \mathrm{TeV}$ and $\kappa=\kappa^{\prime}=0$. Lighter points correspond to smaller values of $\Delta_{\mathrm{BG}}$ and therefore to less tuned points.
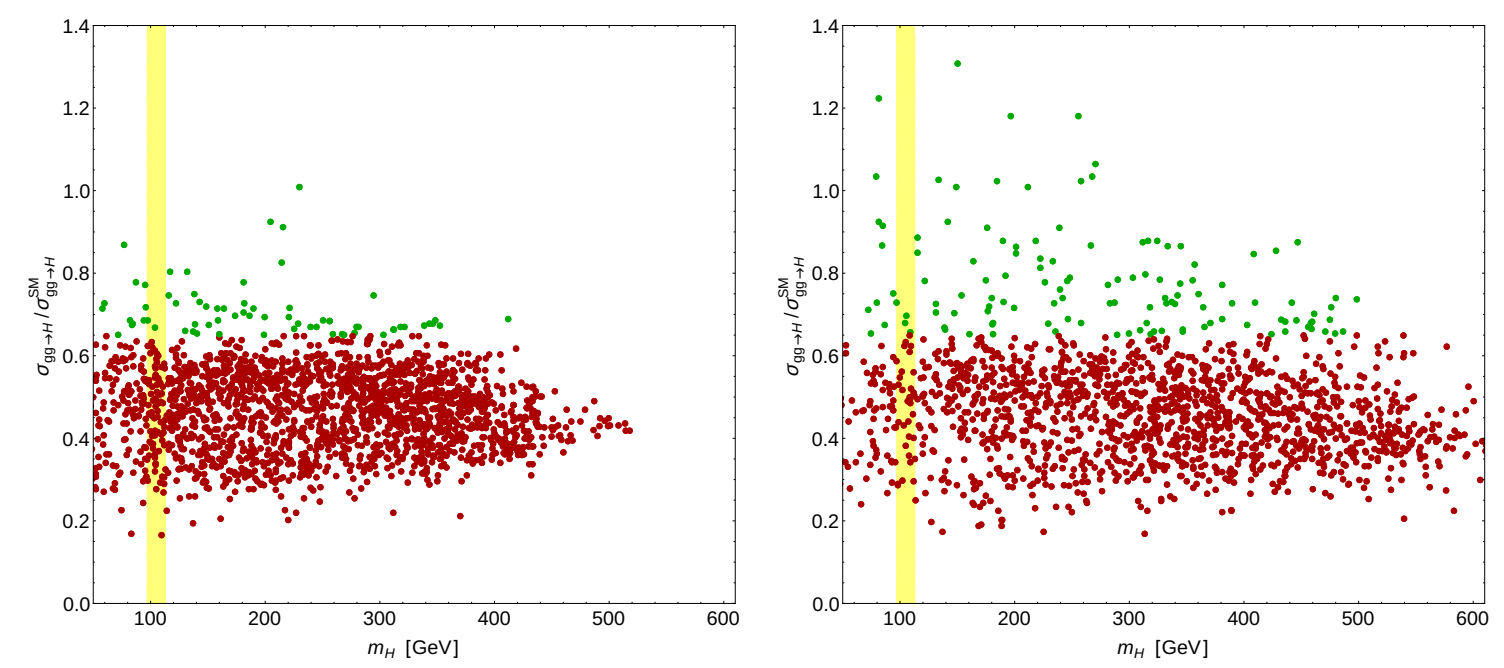

Figure 18. $g g \rightarrow H$ cross section normalized to the SM value as a function of the Higgs mass in the $\mathrm{MCHM}_{14}$ for $Y_{q}^{*}=0.7$ (left) and $Y_{*}^{q}=1.4$ (right). In both cases we have assumed $f_{\pi}=0.8 \mathrm{TeV}$ and $\kappa=\kappa^{\prime}=0$. The red color indicates points that deviate by more than $35 \%$ from the SM prediction.

the significant ad-hoc tuning in this model also becomes apparent. Not only is the generic scale of the Higgs mass raised to $m_{H} \sim 300 \mathrm{GeV}$ and beyond (cf. (2.44)), in particular a Higgs around $m_{H} \sim 100 \mathrm{GeV}$ leads in general to a large tuning of $\Delta_{\mathrm{BG}} \sim \mathcal{O}(50-100)$ in the case of $Y_{q}^{*}=0.7$ (left panel), while $Y_{*}^{q}=1.4$ (right panel) leads generically to $\Delta_{\mathrm{BG}} \sim \mathcal{O}(50-200)$, where we again employed $f_{\pi}=0.8 \mathrm{TeV}$.

Moreover, remember that this model induces large corrections to the SM Higgs couplings, in particular the $g g \rightarrow H$ cross section receives sizable changes, as shown in section 3.1.3. The numerical predictions for $\sigma_{g g \rightarrow H}$, normalized to the SM value, are given in figure 18 with analogous parameters as before. We can see that most of the parameter space features large corrections of more than $35 \%$, as indicated by the red color. Thus, 
this solution to the light-partner issue seems indeed already to be disfavored from Higgs physics at the LHC.

We now move forward to the more economical model of mixing the $t_{L}$ with a $\mathbf{1 4}$, while the $t_{R}$ mixes with a singlet, i.e. the $\mathrm{MCHM}_{14-1}$. In that case, it turns out that one needs brane-localized fermion kinetic terms $\kappa_{B}^{q}, \kappa_{T}^{q}>0$ to allow for a non-vanishing $\alpha_{t}$, see section 3.1.3. Once more we however also expect to be able to lift the top-partner masses beyond the $\mathrm{TeV}$ scale, again at the price of a non-negligible ad-hoc tuning via the sizable quark contribution to $\beta$, which due to the $\mathbf{1}_{R}$ should however again be more moderate than before, comparable to the case of the $\mathrm{mMCHM}_{5-1}^{\mathrm{III}>}$. Indeed, this picture is supported from the $m_{2 / 3}^{\min }$ vs. $m_{H}$ plots presented in figure 19a, where we see that, while still light partners are possible, in a large part of the viable parameter space they are lifted to the $\sim 2 \mathrm{TeV}$ scale, both for $Y_{*}^{q}=0.7$ (left plot) and $Y_{*}^{q}=1.4$ (right plot), where always $f_{\pi}=0.8 \mathrm{TeV}$. Although the density of points in our scan gets slightly more diluted approaching the experimental Higgs mass, compared to the $\mathrm{MCHM}_{5}$, the yellow band can be reached relatively easily with a moderate tuning of $\Delta_{\mathrm{BG}} \sim(30-40)$, comparable to the case of the $\mathrm{mMCHM}_{5-1}^{\mathrm{III}>}$. Nevertheless, the numbers show that this ad-hoc tuning is in general somewhat more severe than the "double tuning" of the $\mathrm{MCHM}_{5}$ or the $\mathrm{mMCHM}_{5}^{\mathrm{III}}$.

We examine this behavior more quantitatively in figure 19b, confronting the fine tuning of the $\mathrm{MCHM}_{14-1}$ and the $\mathrm{mMCHM}_{5-1}^{\mathrm{III}>}$ with that of the $\mathrm{mMCHM}_{5}^{\mathrm{III}}$, which can completely avoid the ad-hoc tuning, employing $Y_{*}^{q}=0.7, f_{\pi}=0.8$, as well as lepton brane masses as before. In the left panel we display the survival function $P_{\Delta}$, describing the fraction of points with a given fine-tuning larger or equal to the value of $\Delta_{\mathrm{BG}}$ on the x-axis, for all points in the viable Higgs-mass band. This confirms clearly that, while the general tuning in both the $\mathrm{mMCHM}_{14-1}$ and the $\mathrm{mMCHM}_{5-1}^{\mathrm{III}>}$ is moderate - $P_{\Delta}$ starts to drop considerably below 1 around $\Delta_{\mathrm{BG}} \gtrsim 25$ - the drop in the mMCHM III already starts at $\Delta_{\mathrm{BG}} \gtrsim 10$, improving the tuning by a factor of 2 to 3 . The difference becomes even more apparent once we use the constraint $m_{2 / 3}^{\min }>1 \mathrm{TeV}$, employed in the right panel of figure 19b, which ensures the absence of light partners. As explained before, this selects the points with the smallest tuning within the $\mathrm{mMCHM}_{5}^{\mathrm{III}}$, opening for the first time the parameter space to allow for a minimal tuning of even less than $10 \%$ while at the same time not predicting ultra-light partners. While already this model provides a motivation for the appearance of a symmetric representation of $\mathrm{SO}(5)$ and does not introduce many new particles, a big virtue of the $\mathrm{mMCHM}_{5-1}^{\mathrm{III}>}$ on the other hand is its highest degree of minimality and naturalness. This is true in the quark sector, embedding each SM fermion in the most minimal $\mathrm{SO}(5)$ multiplet one can think of (respecting custodial protection of the $Z$ couplings), as well as in the lepton sector, where it provides the most minimal realization of the type-III seesaw, leading to the least number of dof in the full fermion sector for viable models. Obviously, raising top partners via this model is possible in a much more minimal way than in the $\mathrm{MCHM}_{14-1}$, which adds many colored dof at the $\mathrm{TeV}$ scale, while the amount of tuning remains the same. 

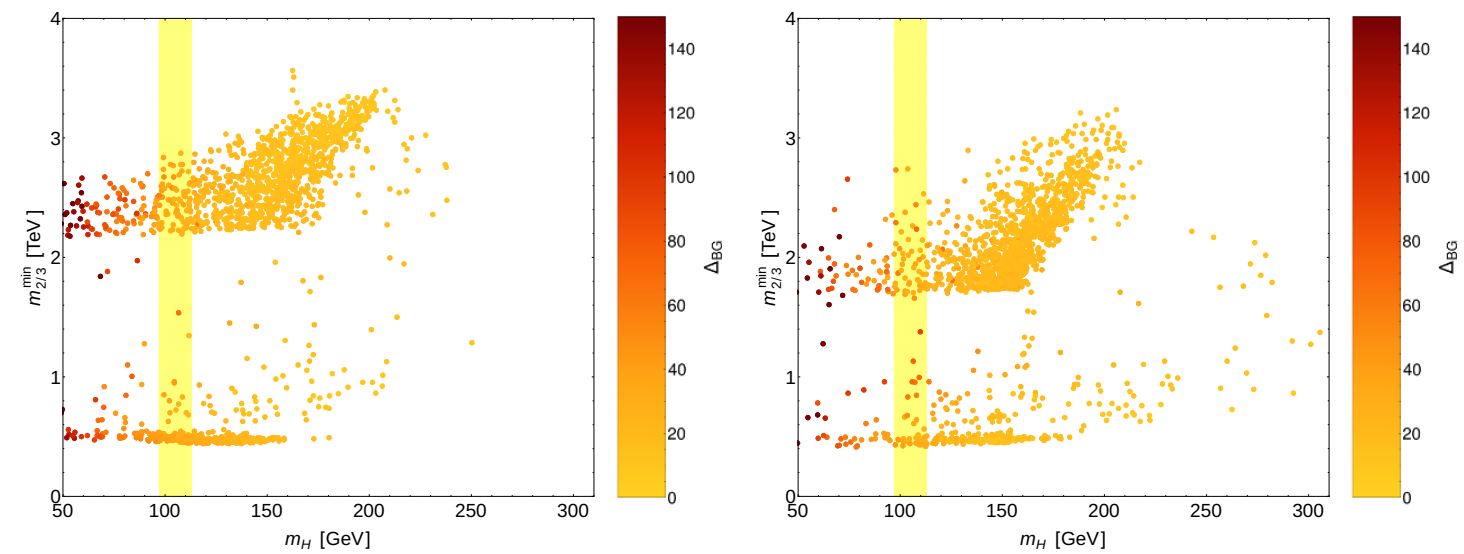

(a) $m_{2 / 3}^{\min }$ vs. $m_{H}$ in the $\operatorname{MCHM}_{14-1}$, with $Y_{*}^{q}=0.7$ (left), $Y_{*}^{q}=1.4$ (right), while $0 \leq \kappa_{B}^{q}, \kappa_{T}^{q} \leq Y_{*}^{q}$. Lighter points correspond to smaller values of $\Delta_{\mathrm{BG}}$ and therefore to less tuned points.
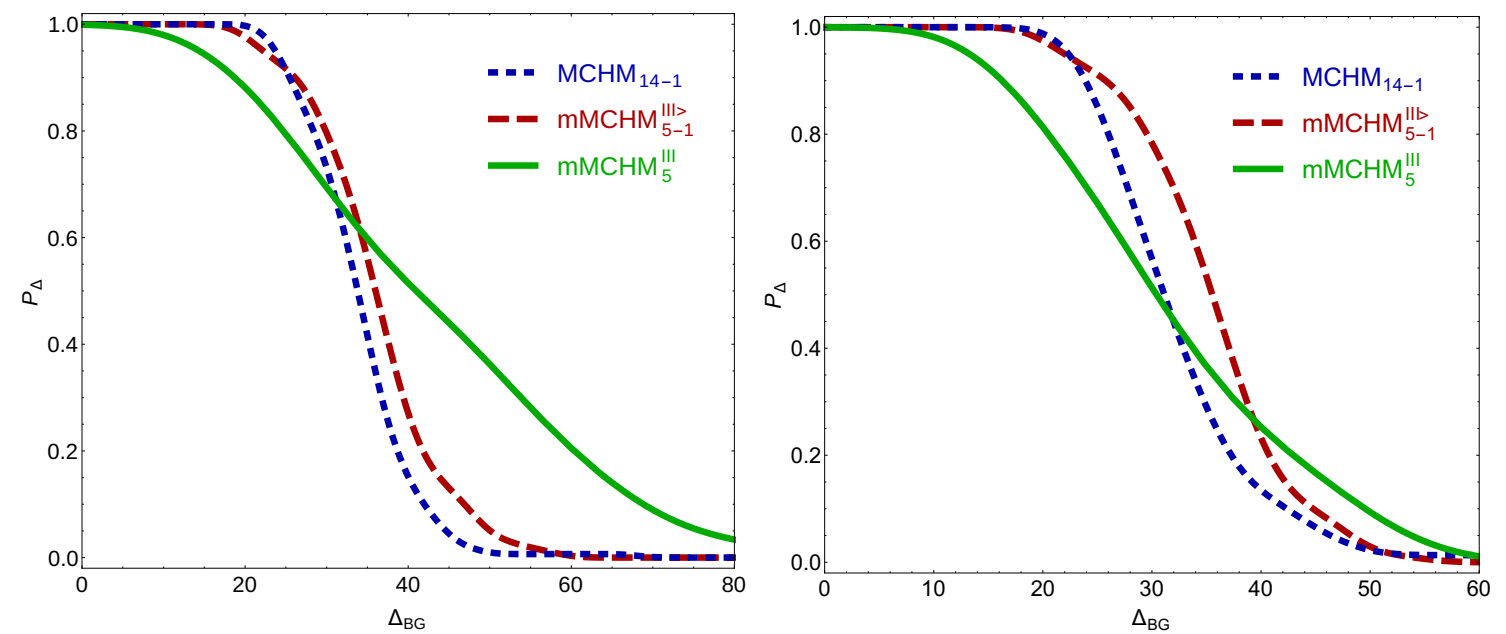

(b) Survival function $\mathcal{P}_{\Delta}$ for the fine tuning $\Delta_{\mathrm{BG}}$ in the $\mathrm{MCHM}_{14-1}$ (dotted blue), $\mathrm{mMCHM}_{5-1}^{\mathrm{III}>}$ with $Y_{*}^{l}=0.7$ (dashed red), and $\mathrm{mMCHM}_{5}^{\mathrm{III}}$ with $Y_{*}^{l}=0.35$ (solid green), always employing $Y_{*}^{q}=0.7$. In the left panel we show the full parameter range in agreement with $m_{H}$, while in the right we require $m_{2 / 3}^{\min }>1 \mathrm{TeV}$.

Figure 19. Fine tuning $\Delta_{\mathrm{BG}}$ in the $\mathrm{MCHM}_{14-1}$ (figure 19a) as well as compared to the minimal models of lepton compositeness (figure 19b). We always employ $\kappa=\kappa^{\prime}=0$ and $f_{\pi}=0.8 \mathrm{TeV}$

\section{Conclusions}

We have presented a comprehensive survey of the impact of different realizations of the fermion sector on the potential of the composite $\mathrm{SO}(5) / \mathrm{SO}(4)$ Higgs. Putting a special emphasis on the lepton sector, mostly neglected in other studies, we presented new models that allow to lift the masses of the lightest top partners well above the region currently probed by the LHC in a way orthogonal to previous studies, i.e. without a significant enlargement of the colored fermion sector and, as such, not sacrificing the prediction of a fully natural light Higgs. In particular, we pointed out the possible minimality of this solution by presenting the $\mathrm{mMCHM}^{\mathrm{III}}$-type models, which, although featuring a symmetric representation of $\mathrm{SO}(5)$, allow for less new particles than e.g. the standard $\mathrm{MCHM}_{5}$, by unifying 
both left-handed and right-handed SM-leptons in a single multiplet, respectively. We also demonstrated how, in contrast to the quark sector, the lepton sector provides a compelling motivation for the emergence of a symmetric representation via the seesaw mechanism and how the $\mathrm{mMCHM}_{5}^{\mathrm{III}}$ allows to reconcile the absence of light partners with a minimal tuning. It is indeed the particular type-III seesaw, explored for the first time, that allows the unification of the right-handed lepton fields - for quarks the $\mathrm{SU}(2)$ breaking masses and/or the quantum numbers do not allow for a similar mechanism. We thus provided the first example of a composite model that can describe a full fermion sector with only two composite fields.

Indeed, in the case of no discovery of light top partners rather soon after the restart of the LHC (with $\mathcal{O}\left(100 \mathrm{fb}^{-1}\right)$ ), this class of models with non-negligible leptonic effects could furnish the only economical composite models that are still compatible with a minimal tuning of not more than $10 \%$. Although here the sizable contribution of the lepton sector to the Higgs mass has a quite different origin from the sizable top quark contribution (i.e., it is not caused by a large $m_{f}$ ), the setup still intertwines the flavor and/or neutrinomass structure with the Higgs potential. In particular, models with pronounced leptonic effects do not suffer from large corrections in Higgs production, which, as we demonstrated explicitly, already challenge the $\mathrm{MCHM}_{14}$. To exclude the bulk of the parameter space of the models featuring a symmetric representation in the lepton sector directly via top partner searches would be challenging, even at the HL-LHC with $3000 \mathrm{fb}^{-1}$, which has a reach of roughly $1.7 \mathrm{TeV}[68,69]$. A $100 \mathrm{TeV}$ collider on the other hand is expected to probe the full parameter space of natural models as discussed here. Indirect detection via the modification of the Higgs couplings, e.g. in channels involving leptons, or the search for color octet resonances could be alternative options to unveil signs of the models. The impact on lepton flavor, which might require some additional model building, will be investigated elsewhere [70]. However, let us already note that all setups considered here feature a first layer of flavor protection since the right-handed leptons come with a custodial protection due to the $P_{L R}$ symmetry, while the left-handed ones are rather strongly UV localized, i.e., they feature a very small compositeness. Since the lepton sector, which needs to be included in a fully realistic composite model, allows to construct minimal models with minimal tuning and a naturally light Higgs, that do not predict light top partners, the absence of corresponding LHC signals should not threaten the idea of the minimal composite Higgs.

\section{Acknowledgments}

We are grateful to Roberto Contino, Giuliano Panico, and José Santiago for useful discussions. The research of the authors was supported by the Swiss National Foundation under contract SNF 200021-143781. The research of FG was also supported by a Marie Curie Intra European Fellowship within the 7th European Community Framework Programme (grant no. PIEF-GA-2013-628224). 


\section{A Generators and explicit form of Goldstone matrix}

The generators of $\mathrm{SO}(5)$ in the fundamental representation read

$$
\begin{array}{rlrl}
T_{L, i j}^{a} & =-\frac{i}{2}\left[\frac{1}{2} \epsilon^{a b c}\left(\delta_{i}^{b} \delta_{j}^{c}-\delta_{j}^{b} \delta_{i}^{c}\right)+\left(\delta_{i}^{a} \delta_{j}^{4}-\delta_{j}^{a} \delta_{i}^{4}\right)\right], & & a=1,2,3, \\
T_{R, i j}^{a} & =-\frac{i}{2}\left[\frac{1}{2} \epsilon^{a b c}\left(\delta_{i}^{b} \delta_{j}^{c}-\delta_{j}^{b} \delta_{i}^{c}\right)-\left(\delta_{i}^{a} \delta_{j}^{4}-\delta_{j}^{a} \delta_{i}^{4}\right)\right], & & a=1,2,3, \\
T_{i j}^{\hat{a}} & =-\frac{i}{\sqrt{2}}\left[\delta_{i}^{\hat{a}} \delta_{j}^{5}-\delta_{j}^{\hat{a}} \delta_{i}^{5}\right], \quad \hat{a}=1,2,3,4, &
\end{array}
$$

which have been chosen orthonormal with respect to the Cartan-Killing inner product, i.e. $\operatorname{Tr}\left(T^{\alpha} \cdot T^{\beta}\right)=\delta^{\alpha \beta}$, for all $T^{\alpha}, T^{\beta} \in\left\{T_{L}^{a}, T_{R}^{a}, T^{\hat{a}}\right\}$. As $\left[T_{L}^{3}, T_{R}^{3}\right]=0$ we can go to a new basis where both generators are diagonal at the same time. This will make the explicit form of the fermion representations much simpler. To this end, we perform the rotation

$$
T \rightarrow \mathbf{A} \cdot T \cdot \mathbf{A}^{\dagger}, \quad \text { with } T \in\left\{T_{L}^{a}, T_{R}^{a}, T^{\hat{a}}\right\},
$$

where $\mathbf{A}$ is the unitary matrix

$$
\mathbf{A}=\frac{1}{\sqrt{2}}\left(\begin{array}{ccccc}
1 & -i & 0 & 0 & 0 \\
0 & 0 & -i & 1 & 0 \\
0 & 0 & i & 1 & 0 \\
-1 & -i & 0 & 0 & 0 \\
0 & 0 & 0 & 0 & \sqrt{2}
\end{array}\right)
$$

The previous set of generators, together with the identity, can be extended to span a basis of the linear space of square matrices of order $n$ over the complex field $\mathbb{C}, F L(n, \mathbb{C})$. In particular, in the basis of (A.1), this can be done by adding the following set of matrices

$$
\begin{aligned}
\tilde{T}_{i j}^{\hat{a}} & =\frac{1}{\sqrt{2}}\left[\delta_{i}^{\hat{a}} \delta_{j}^{5}+\delta_{j}^{\hat{a}} \delta_{i}^{5}\right], & & \hat{a}=1,2,3,4, \\
\tilde{T}_{i j}^{\hat{a} \hat{b}} & =\frac{1}{\sqrt{2}}\left[\delta_{i}^{\hat{a}} \delta_{j}^{\hat{b}}+\delta_{j}^{\hat{a}} \delta_{i}^{\hat{b}}\right], & & \hat{a}<\hat{b} \in\{1,2,3,4\}, \\
\tilde{T}_{i j}^{a a} & =\delta_{i}^{\hat{a}} \delta_{j}^{\hat{a}}+\delta_{i}^{4} \delta_{j}^{4}-\frac{1}{2} \delta_{i j}+\frac{1}{2} \delta_{i}^{5} \delta_{j}^{5}, & & a=1,2,3, \\
\tilde{T}_{i j}^{s} & =\frac{1}{2 \sqrt{5}} \operatorname{diag}(1,1,1,1,-4), & &
\end{aligned}
$$

that can be collectively denoted by $\hat{T}^{A} \in\left\{\tilde{T}^{\hat{a} \hat{b}}, \tilde{T}^{a a}, \tilde{T}^{\hat{a}}, \tilde{T}^{s}\right\}$.

\section{B Fermion representations}

\section{B.1 Fundamental}

The fundamental representation of $\mathrm{SO}(5)$ decompose under $\mathrm{SO}(4) \cong \mathrm{SU}(2)_{L} \times \mathrm{SU}(2)_{R}$ as $\mathbf{5}=(\mathbf{2}, \mathbf{2}) \oplus(\mathbf{1}, \mathbf{1})$. More explictly, in the basis given by the transformation (A.2), a field $\chi_{5}$ transforming under this representation will read

$$
\chi_{\mathbf{5}}=\chi^{(\mathbf{2 , 2})}+\chi^{(\mathbf{1 , 1})}
$$


with

$$
\chi^{(\mathbf{2 , 2})}=\left(\begin{array}{c}
\chi_{++}^{(\mathbf{2 , 2}} \\
\chi_{-+}^{(\mathbf{2}, 2)} \\
\chi_{+}^{(\mathbf{2 , 2})} \\
\chi_{--}^{(\mathbf{2 , 2})} \\
0
\end{array}\right), \quad \chi^{(\mathbf{1 , 1})}=\left(\begin{array}{c}
0 \\
0 \\
0 \\
0 \\
\chi_{s}^{(\mathbf{1}, \mathbf{1})}
\end{array}\right)
$$

In the above equation the first and second subscripts \pm on the $\chi^{(\mathbf{2 , 2})}$ fields correspond to $T_{L}^{3}= \pm 1 / 2$ and $T_{R}^{3}= \pm 1 / 2$, respectively.

\section{B.2 Adjoint}

Similarly, the adjoint representation of $\mathrm{SO}(5)$ decomposes as $\mathbf{1 0}=(\mathbf{3}, \mathbf{1}) \oplus(\mathbf{1}, \mathbf{3}) \oplus(\mathbf{2}, \mathbf{2})$. Still in the same basis, a field $\chi_{\mathbf{1 0}}$ transforming under this representation will read

$$
\chi_{10}=\chi^{(\mathbf{3 , 1})}+\chi^{(\mathbf{1 , 3})}+\chi^{(\mathbf{2 , 2})},
$$

where

$$
\begin{aligned}
& \chi^{(\mathbf{3}, \mathbf{1})}=\left(\begin{array}{ccccc}
\frac{1}{2} \chi_{0}^{(\mathbf{3}, \mathbf{1})} & -\frac{i}{\sqrt{2}} \chi_{+}^{(\mathbf{3}, \mathbf{1})} & 0 & 0 & 0 \\
\frac{i}{\sqrt{2}} \chi_{-}^{(\mathbf{3}, \mathbf{1})} & -\frac{1}{2} \chi_{0}^{(\mathbf{3}, \mathbf{1})} & 0 & 0 & 0 \\
0 & 0 & \frac{1}{2} \chi_{0}^{(\mathbf{3}, \mathbf{1})} & -\frac{i}{\sqrt{2}} \chi_{+}^{(\mathbf{3}, \mathbf{1})} & 0 \\
0 & 0 & \frac{i}{\sqrt{2}} \chi_{-}^{(\mathbf{3}, \mathbf{1})} & -\frac{1}{2} \chi_{0}^{(\mathbf{3}, 1)} & 0 \\
0 & 0 & 0 & 0 & 0
\end{array}\right), \\
& \chi^{(\mathbf{1 , 3})}=\left(\begin{array}{ccccc}
\frac{1}{2} \chi_{0}^{(\mathbf{1 , 3})} & 0 & \frac{i}{\sqrt{2}} \chi_{+}^{(\mathbf{1 , 3})} & 0 & 0 \\
0 & \frac{1}{2} \chi_{0}^{(\mathbf{1 , 3})} & 0 & \frac{i}{\sqrt{2}} \chi_{+}^{(\mathbf{1 , 3})} & 0 \\
-\frac{i}{\sqrt{2}} \chi_{-}^{(\mathbf{1}, \mathbf{3})} & 0 & -\frac{1}{2} \chi_{0}^{(\mathbf{1}, \mathbf{3})} & 0 & 0 \\
0 & -\frac{i}{\sqrt{2}} \chi_{-}^{(\mathbf{1}, \mathbf{3})} & 0 & -\frac{1}{2} \chi_{0}^{(\mathbf{1}, \mathbf{3})} & 0 \\
0 & 0 & 0 & 0 & 0
\end{array}\right) \\
& \chi^{(\mathbf{2 , 2})}=\frac{1}{\sqrt{2}}\left(\begin{array}{ccccc}
0 & 0 & 0 & 0 & -i \chi_{++}^{(\mathbf{2 , 2}} \\
0 & 0 & 0 & 0 & -\chi_{-+}^{(\mathbf{2 , 2})} \\
0 & 0 & 0 & 0 & \chi_{+-}^{(\mathbf{2 , 2})} \\
0 & 0 & 0 & 0 & i \chi_{--}^{(\mathbf{2 , 2})} \\
i \chi_{--}^{(\mathbf{2 , 2})}-\chi_{+-}^{(\mathbf{2 , 2})} & \chi_{-+}^{(\mathbf{2 , 2})}-i \chi_{++}^{(\mathbf{2 , 2})} & 0
\end{array}\right),
\end{aligned}
$$

and the subscripts, \pm 0 on $\chi^{(\mathbf{3 , 1})}\left(\chi^{(\mathbf{1 , 3})}\right)$ correspond to $T_{L}^{3}= \pm 1,0\left(T_{R}^{3}= \pm 1,0\right)$, respectively.

\section{B.3 Symmetric}

Finally, we consider the symmetric representation of $\mathrm{SO}(5), \mathbf{1 4}$, decomposing under $\mathrm{SO}(4)$ as $\mathbf{1 4}=(\mathbf{1}, \mathbf{1}) \oplus(\mathbf{2}, \mathbf{2}) \oplus(\mathbf{3}, \mathbf{3})$. A field $\chi_{\mathbf{1 4}}$ transforming under this representation can be 
expressed in the basis given by (A.2) as

$$
\chi_{\mathbf{1 4}}=\chi^{(\mathbf{0 , 0})}+\chi^{(\mathbf{2 , 2})}+\chi^{(\mathbf{3 , 3})},
$$

with

$$
\begin{aligned}
\chi^{(\mathbf{0 , 0})}=\chi_{s}^{(\mathbf{0 , 0})} \frac{1}{2 \sqrt{5}} \operatorname{diag}(1,1,1,1,-4), \\
\chi^{(\mathbf{2 , 2})}=\frac{1}{\sqrt{2}}\left(\begin{array}{ccccc}
0 & 0 & 0 & 0 & \chi_{++}^{(\mathbf{2 , 2}} \\
0 & 0 & 0 & 0 & \chi_{-+}^{(\mathbf{2 , 2})} \\
0 & 0 & 0 & 0 & -\chi_{+-}^{(\mathbf{2}, \mathbf{2}} \\
0 & 0 & 0 & 0 & -\chi_{--}^{(\mathbf{2 , 2})} \\
\chi_{--}^{(\mathbf{2 , 2})} & -\chi_{+-}^{(\mathbf{2 , 2})} & \chi_{-+}^{(\mathbf{2 , 2})} & -\chi_{++}^{(\mathbf{2 , 2})} & 0
\end{array}\right),
\end{aligned}
$$

and

$$
\chi^{(\mathbf{3}, \mathbf{3})}=\left(\begin{array}{ccccc}
-\frac{1}{2} \chi_{00}^{(\mathbf{3}, \mathbf{3})} & \frac{1}{\sqrt{2}} \chi_{+0}^{(\mathbf{3}, \mathbf{3})} & \frac{1}{\sqrt{2}} \chi_{0+}^{(\mathbf{3}, \mathbf{3})} & -\chi_{++}^{(\mathbf{3}, \mathbf{3})} & 0 \\
-\frac{1}{\sqrt{2}} \chi_{-0}^{(\mathbf{3}, \mathbf{3})} & \frac{1}{2} \chi_{00}^{(\mathbf{3}, \mathbf{3})} & \chi_{-+}^{(\mathbf{3}, \mathbf{3})} & -\frac{1}{\sqrt{2}} \chi_{0+}^{(\mathbf{3}, \mathbf{3})} & 0 \\
-\frac{1}{\sqrt{2}} \chi_{0-}^{(\mathbf{3}, \mathbf{3})} & -\chi_{+-}^{(\mathbf{3}, \mathbf{3})} & \frac{1}{2} \chi_{00}^{(\mathbf{3}, \mathbf{3})} & -\frac{1}{\sqrt{2}} \chi_{+0}^{(\mathbf{3}, \mathbf{3})} & 0 \\
\chi_{--}^{(\mathbf{3}, \mathbf{3})} & \frac{1}{\sqrt{2}} \chi_{0-}^{(\mathbf{3}, \mathbf{3})} & \frac{1}{\sqrt{2}} \chi_{-0}^{(\mathbf{3}, \mathbf{3})} & -\frac{1}{2} \chi_{00}^{(\mathbf{3}, \mathbf{3})} & 0 \\
0 & 0 & 0 & 0 & 0
\end{array}\right)
$$

Analagously, the first and second subscripts, \pm 0 in $\chi^{(\mathbf{3 , 3})}$ correspond to $T_{L}^{3}= \pm 1,0$ and $T_{R}^{3}= \pm 1,0$, respectively.

Open Access. This article is distributed under the terms of the Creative Commons Attribution License (CC-BY 4.0), which permits any use, distribution and reproduction in any medium, provided the original author(s) and source are credited.

\section{References}

[1] ATLAS collaboration, Observation of a new particle in the search for the Standard Model Higgs boson with the ATLAS detector at the LHC, Phys. Lett. B 716 (2012) 1 [arXiv: 1207.7214] [INSPIRE].

[2] CMS collaboration, Observation of a new boson at a mass of $125 \mathrm{GeV}$ with the CMS experiment at the LHC, Phys. Lett. B 716 (2012) 30 [arXiv:1207.7235] [INSPIRE].

[3] R. Contino, L. Da Rold and A. Pomarol, Light custodians in natural composite Higgs models, Phys. Rev. D 75 (2007) 055014 [hep-ph/0612048] [INSPIRE].

[4] A.D. Medina, N.R. Shah and C.E.M. Wagner, Gauge-Higgs unification and radiative electroweak symmetry breaking in warped extra dimensions, Phys. Rev. D 76 (2007) 095010 [arXiv: 0706.1281] [INSPIRE].

[5] C. Csáki, A. Falkowski and A. Weiler, The flavor of the composite pseudo-Goldstone Higgs, JHEP 09 (2008) 008 [arXiv: 0804.1954] [INSPIRE]. 
[6] S. De Curtis, M. Redi and A. Tesi, The 4D composite Higgs, JHEP 04 (2012) 042 [arXiv:1110.1613] [INSPIRE].

[7] O. Matsedonskyi, G. Panico and A. Wulzer, Light top partners for a light composite Higgs, JHEP 01 (2013) 164 [arXiv:1204.6333] [INSPIRE].

[8] D. Marzocca, M. Serone and J. Shu, General composite Higgs models, JHEP 08 (2012) 013 [arXiv: 1205.0770] [INSPIRE].

[9] A. Pomarol and F. Riva, The composite Higgs and light resonance connection, JHEP 08 (2012) 135 [arXiv:1205.6434] [INSPIRE].

[10] G. Panico, M. Redi, A. Tesi and A. Wulzer, On the tuning and the mass of the composite Higgs, JHEP 03 (2013) 051 [arXiv: 1210.7114] [INSPIRE].

[11] P.R. Archer, Fine tuning in the holographic minimal composite Higgs model, arXiv: 1403.8048 [INSPIRE].

[12] F. del Aguila, A. Carmona and J. Santiago, Neutrino masses from an $A_{4}$ symmetry in holographic composite Higgs models, JHEP 08 (2010) 127 [arXiv:1001.5151] [INSPIRE].

[13] A. Carmona and F. Goertz, Composite taus and Higgs decays, PoS (EPS-HEP 2013) 267 [arXiv: 1310.3825] [INSPIRE].

[14] A. Carmona and F. Goertz, Custodial leptons and Higgs decays, JHEP 04 (2013) 163 [arXiv: 1301.5856] [INSPIRE].

[15] G.F. Giudice, C. Grojean, A. Pomarol and R. Rattazzi, The strongly-interacting light Higgs, JHEP 06 (2007) 045 [hep-ph/0703164] [INSPIRE].

[16] C. Anastasiou, E. Furlan and J. Santiago, Realistic composite Higgs models, Phys. Rev. D 79 (2009) 075003 [arXiv: 0901.2117 ] [INSPIRE].

[17] G. Panico and A. Wulzer, The discrete composite Higgs model, JHEP 09 (2011) 135 [arXiv: 1106.2719] [INSPIRE].

[18] A. Azatov and J. Galloway, Light custodians and Higgs physics in composite models, Phys. Rev. D 85 (2012) 055013 [arXiv:1110.5646] [INSPIRE].

[19] N.S. Manton, A new six-dimensional approach to the Weinberg-Salam model, Nucl. Phys. B 158 (1979) 141 [INSPIRE].

[20] H. Hatanaka, T. Inami and C.S. Lim, The gauge hierarchy problem and higher dimensional gauge theories, Mod. Phys. Lett. A 13 (1998) 2601 [hep-th/9805067] [INSPIRE].

[21] G. von Gersdorff, N. Irges and M. Quirós, Bulk and brane radiative effects in gauge theories on orbifolds, Nucl. Phys. B 635 (2002) 127 [hep-th/0204223] [INSPIRE].

[22] C. Csáki, C. Grojean and H. Murayama, Standard model Higgs from higher dimensional gauge fields, Phys. Rev. D 67 (2003) 085012 [hep-ph/0210133] [INSPIRE].

[23] R. Contino, Y. Nomura and A. Pomarol, Higgs as a holographic pseudo-Goldstone boson, Nucl. Phys. B 671 (2003) 148 [hep-ph/0306259] [InSPIRE].

[24] K. Agashe, R. Contino and A. Pomarol, The minimal composite Higgs model, Nucl. Phys. B 719 (2005) 165 [hep-ph/0412089] [INSPIRE].

[25] H. Terazawa, K. Akama and Y. Chikashige, Unified model of the Nambu-Jona-Lasinio type for all elementary particle forces, Phys. Rev. D 15 (1977) 480 [INSPIRE].

[26] H. Terazawa, Subquark model of leptons and quarks, Phys. Rev. D 22 (1980) 184 [INSPIRE]. 
[27] S. Dimopoulos and J. Preskill, Massless composites with massive constituents, Nucl. Phys. B 199 (1982) 206 [INSPIRE].

[28] D.B. Kaplan and H. Georgi, SU(2) $\times$ U(1) breaking by vacuum misalignment, Phys. Lett. B 136 (1984) 183 [INSPIRE].

[29] D.B. Kaplan, H. Georgi and S. Dimopoulos, Composite Higgs scalars, Phys. Lett. B 136 (1984) 187 [INSPIRE].

[30] H. Georgi, D.B. Kaplan and P. Galison, Calculation of the composite Higgs mass, Phys. Lett. B 143 (1984) 152 [INSPIRE].

[31] T. Banks, Constraints on $\mathrm{SU}(2) \times \mathrm{U}(1)$ breaking by vacuum misalignment, Nucl. Phys. B 243 (1984) 125 [INSPIRE].

[32] H. Georgi and D.B. Kaplan, Composite Higgs and custodial SU(2), Phys. Lett. B 145 (1984) 216 [INSPIRE].

[33] M.J. Dugan, H. Georgi and D.B. Kaplan, Anatomy of a composite Higgs model, Nucl. Phys. B 254 (1985) 299 [INSPIRE].

[34] S.R. Coleman, J. Wess and B. Zumino, Structure of phenomenological Lagrangians. 1, Phys. Rev. 177 (1969) 2239 [INSPIRE].

[35] C.G. Callan Jr., S.R. Coleman, J. Wess and B. Zumino, Structure of phenomenological Lagrangians. 2, Phys. Rev. 177 (1969) 2247 [INSPIRE].

[36] K. Agashe, A. Delgado, M.J. May and R. Sundrum, RS1, custodial isospin and precision tests, JHEP 08 (2003) 050 [hep-ph/0308036] [INSPIRE].

[37] K. Agashe, R. Contino, L. Da Rold and A. Pomarol, A custodial symmetry for Z $b \bar{b}$, Phys. Lett. B 641 (2006) 62 [hep-ph/0605341] [INSPIRE].

[38] M. Carena, E. Ponton, J. Santiago and C.E.M. Wagner, Light Kaluza Klein states in Randall-Sundrum models with custodial SU(2), Nucl. Phys. B 759 (2006) 202 [hep-ph/0607106] [INSPIRE].

[39] M. Carena, E. Ponton, J. Santiago and C.E.M. Wagner, Electroweak constraints on warped models with custodial symmetry, Phys. Rev. D 76 (2007) 035006 [hep-ph/0701055] [INSPIRE].

[40] M. Carena, A.D. Medina, B. Panes, N.R. Shah and C.E.M. Wagner, Collider phenomenology of gauge-Higgs unification scenarios in warped extra dimensions, Phys. Rev. D 77 (2008) 076003 [arXiv: 0712.0095] [inSPIRE].

[41] A. Pomarol and J. Serra, Top quark compositeness: feasibility and implications, Phys. Rev. D 78 (2008) 074026 [arXiv:0806.3247] [INSPIRE].

[42] G. Panico, M. Safari and M. Serone, Simple and realistic composite Higgs models in flat extra dimensions, JHEP 02 (2011) 103 [arXiv:1012.2875] [INSPIRE].

[43] D.B. Kaplan, Flavor at SSC energies: a new mechanism for dynamically generated fermion masses, Nucl. Phys. B 365 (1991) 259 [inSPIRE].

[44] M. Carena, L. Da Rold and E. Pontón, Minimal composite Higgs models at the LHC, JHEP 06 (2014) 159 [arXiv: 1402 .2987] [INSPIRE].

[45] J.M. Maldacena, The large- $N$ limit of superconformal field theories and supergravity, Int. J. Theor. Phys. 38 (1999) 1113 [hep-th/9711200] [INSPIRE]. 
[46] S.S. Gubser, I.R. Klebanov and A.M. Polyakov, Gauge theory correlators from noncritical string theory, Phys. Lett. B 428 (1998) 105 [hep-th/9802109] [INSPIRE].

[47] E. Witten, Anti-de Sitter space and holography, Adv. Theor. Math. Phys. 2 (1998) 253 [hep-th/9802150] [INSPIRE].

[48] N. Arkani-Hamed, M. Porrati and L. Randall, Holography and phenomenology, JHEP 08 (2001) 017 [hep-th/0012148] [INSPIRE].

[49] A. Falkowski, About the holographic pseudo-Goldstone boson, Phys. Rev. D 75 (2007) 025017 [hep-ph/0610336] [INSPIRE].

[50] R. Contino and A. Pomarol, Holography for fermions, JHEP 11 (2004) 058 [hep-th/0406257] [INSPIRE].

[51] B. Batell and T. Gherghetta, Holographic mixing quantified, Phys. Rev. D 76 (2007) 045017 [arXiv:0706.0890] [INSPIRE].

[52] B. Batell and T. Gherghetta, Warped phenomenology in the holographic basis, Phys. Rev. D 77 (2008) 045002 [arXiv:0710.1838] [INSPIRE].

[53] T. Gherghetta, TASI lectures on a holographic view of beyond the standard model physics, arXiv: 1008.2570 [INSPIRE].

[54] F. Goertz, Indirect handle on the down-quark Yukawa coupling, Phys. Rev. Lett. 113 (2014) 261803 [arXiv: 1406.0102 ] [INSPIRE].

[55] CMS collaboration, Inclusive search for a vector-like $T$ quark with charge $2 / 3$ in pp collisions at $\sqrt{s}=8 \mathrm{TeV}$, Phys. Lett. B 729 (2014) 149 [arXiv:1311.7667] [INSPIRE].

[56] D. Pappadopulo, A. Thamm and R. Torre, A minimally tuned composite Higgs model from an extra dimension, JHEP 07 (2013) 058 [arXiv: 1303.3062] [INSPIRE].

[57] M. Carena, A.D. Medina, N.R. Shah and C.E.M. Wagner, Gauge-Higgs unification, neutrino masses and dark matter in warped extra dimensions, Phys. Rev. D 79 (2009) 096010 [arXiv:0901.0609] [INSPIRE].

[58] C. Hagedorn and M. Serone, Leptons in holographic composite Higgs models with non-Abelian discrete symmetries, JHEP 10 (2011) 083 [arXiv:1106.4021] [INSPIRE].

[59] C. Hagedorn and M. Serone, General lepton mixing in holographic composite Higgs models, JHEP 02 (2012) 077 [arXiv:1110.4612] [INSPIRE].

[60] B. Keren-Zur et al., On partial compositeness and the CP asymmetry in charm decays, Nucl. Phys. B 867 (2013) 394 [arXiv: 1205.5803] [INSPIRE].

[61] Y. Hosotani and M. Mabe, Higgs boson mass and electroweak-gravity hierarchy from dynamical gauge-Higgs unification in the warped spacetime, Phys. Lett. B 615 (2005) 257 [hep-ph/0503020] [INSPIRE].

[62] A. Falkowski, Pseudo-Goldstone Higgs production via gluon fusion, Phys. Rev. D 77 (2008) 055018 [arXiv: 0711.0828] [INSPIRE].

[63] R. Foot, H. Lew, X.G. He and G.C. Joshi, Seesaw neutrino masses induced by a triplet of leptons, Z. Phys. C 44 (1989) 441 [inSPIRE].

[64] P.S. Bhupal Dev, D.K. Ghosh, N. Okada and I. Saha, 125 GeV Higgs boson and the type-II seesaw model, JHEP 03 (2013) 150 [arXiv:1301.3453] [INSPIRE]. 
[65] K.-Y. Oda and A. Weiler, Wilson lines in warped space: dynamical symmetry breaking and restoration, Phys. Lett. B 606 (2005) 408 [hep-ph/0410061] [INSPIRE].

[66] M. Baak et al., The electroweak fit of the standard model after the discovery of a new boson at the LHC, Eur. Phys. J. C 72 (2012) 2205 [arXiv:1209.2716] [InSPIRE].

[67] R. Barbieri and G.F. Giudice, Upper bounds on supersymmetric particle masses, Nucl. Phys. B 306 (1988) 63 [INSPIRE].

[68] Y. Gershtein et al., Working group report: new particles, forces and dimensions, arXiv: 1311.0299 [INSPIRE].

[69] Top Quark Working Group collaboration, K. Agashe et al., Working group report: top quark, arXiv:1311.2028 [INSPIRE].

[70] A. Carmona and F. Goertz, in preparation. 